\title{
Diagnostic reference frames for epileptic seizures
}

\author{
Citation for published version (APA):
}

van Ast, J. F. (2005). Diagnostic reference frames for epileptic seizures. [Doctoral Thesis, Maastricht University]. Maastricht University. https://doi.org/10.26481/dis.20050908ja

Document status and date:

Published: 01/01/2005

DOI:

10.26481/dis.20050908ja

Document Version:

Publisher's PDF, also known as Version of record

\section{Please check the document version of this publication:}

- A submitted manuscript is the version of the article upon submission and before peer-review. There can be important differences between the submitted version and the official published version of record.

People interested in the research are advised to contact the author for the final version of the publication, or visit the DOI to the publisher's website.

- The final author version and the galley proof are versions of the publication after peer review.

- The final published version features the final layout of the paper including the volume, issue and page numbers.

Link to publication

\footnotetext{
General rights rights.

- You may freely distribute the URL identifying the publication in the public portal. please follow below link for the End User Agreement:

www.umlib.nl/taverne-license

Take down policy

If you believe that this document breaches copyright please contact us at:

repository@maastrichtuniversity.nl

providing details and we will investigate your claim.
}

Copyright and moral rights for the publications made accessible in the public portal are retained by the authors and/or other copyright owners and it is a condition of accessing publications that users recognise and abide by the legal requirements associated with these

- Users may download and print one copy of any publication from the public portal for the purpose of private study or research.

- You may not further distribute the material or use it for any profit-making activity or commercial gain

If the publication is distributed under the terms of Article $25 \mathrm{fa}$ of the Dutch Copyright Act, indicated by the "Taverne" license above, 


\title{
Diagnostic Reference Frames for EPILEPTIC SEIZURES
}

\author{
Proefschrift
}

ter verkrijging van de graad van doctor aan de Universiteit Maastricht, op gezag van de Rector Magnificus, Prof. Mr. G.P.M.F. Mols

volgens het besluit van het College van Decanen,

in het openbaar te verdedigen

op donderdag 8 seprember 2005 om 16.00 uur

door

Johanna Francina van Ast

geboren op 01 januari 1974

te Rotterdam 


\section{Promotories}

Prof. dr. ir. A. Hasman

Academisch Medisch Centrum, Amsterdam

Prof. dr. W.O. Renier

\section{Co-promotor}

Dr. ir. J.L. Talmon

\section{Beoordelingscommissie}

Prof. dr. M. Limburg (voorzitter)

Prof. dr. B. Aldenkamp

Prof, dr W. van den Bosch

Universitair Medisch Centrum St. Radboud, Nijmegen

Prof. dr. J. van der Lei

Erasmus Medisch Centrum, Rotterdam

Dr. L. Schuwitth

The study presented in this thesis was performed at the Care and Public Health Research Institure (CAPHRI), which participates in the Netherlands School of Primary Care Research (CaRe), acknowledged in 1995 by the Royal Netherlands Academy of Arts and Sciences (KNAW).

The study was made possible by a grant from the Nationaal Epilepsie Fonds (NEF). 
ISBN $90-8590-002 \cdot 6$

(C) Wanda van Ast, Maastricht 2005

Lay-out and cover design D\&L Graphics, www.dlgraphics.nl

Printed by Schrijen-Lipperts-Huntjens

Printing of this thesis was financially supported by Stichting BAZIS and Medecs. 
Chapter 1 General introduction

PART 1: METHODOLOGY

Chapter 2 Inter-participant agreement in the selection of symptoms

Chapter 3 Are seizure descriptions discriminative?

Chapter 4 An approach to knowledge base construction

Part 2: KNOWLEDGE ElICITATION

Chapter 5 Knowledge elicitation among experts

\section{Part 3: VAlidation}

Chapter 6 Validation of the diagnostic reference frames for seizures

Chapter 7 Completeness and accuracy in reporting seizure manifestations

Chapter 8 General discussion

Appendices

Summary

Samenvatting

Dankwoord

Publications 



\section{Chapter 1}

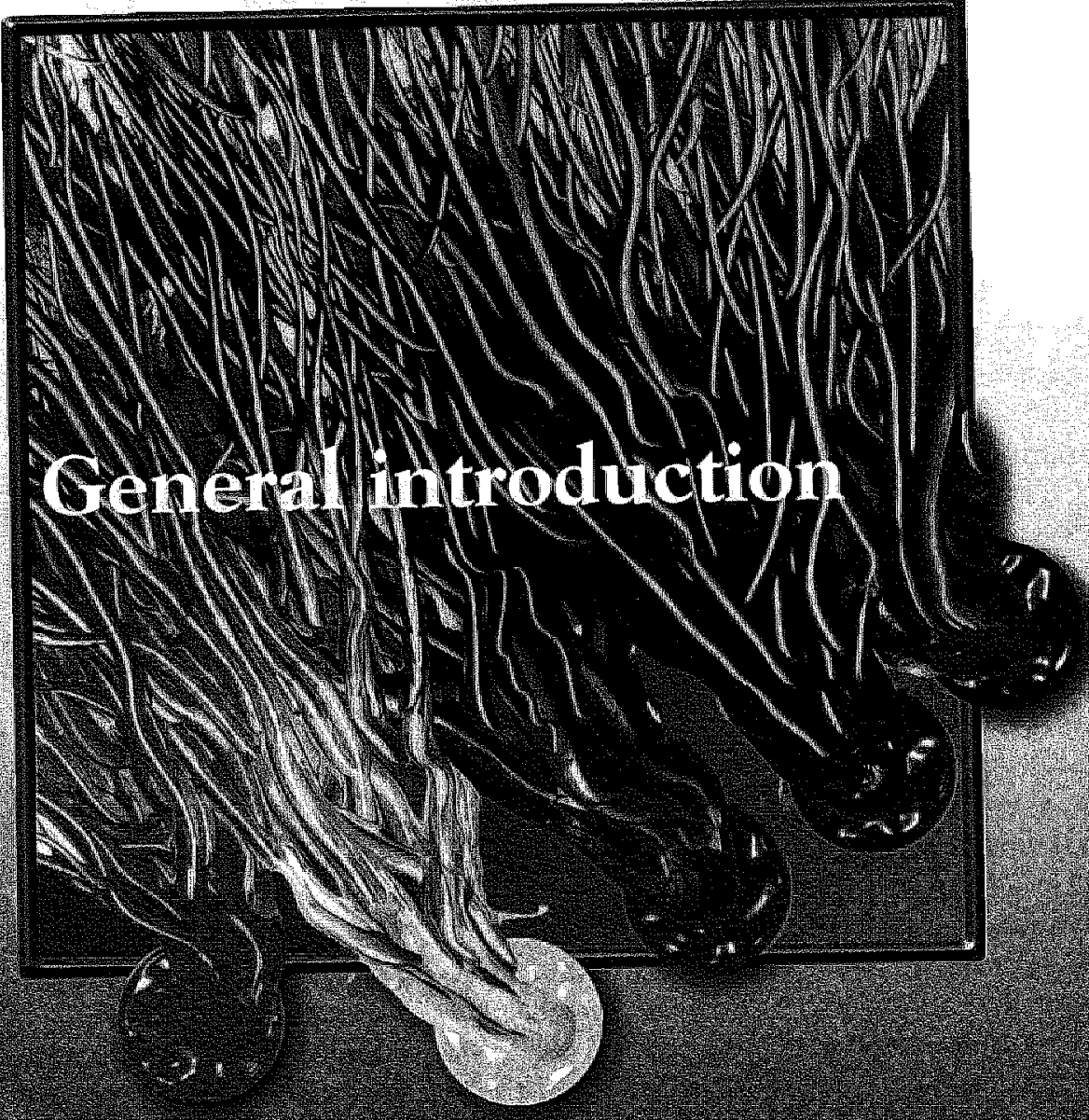




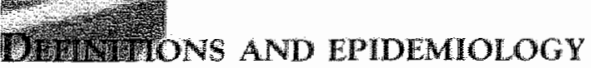

An epillepric seizure is defined as "a clinical manifestation presumed to result from an abnormal and excessive discharge of a set of neurons in the brain. The clinical manifestation consists of sudden and transitory abnormal phenomena which may include alterations of consciousness, motor, sensory, autonomic or psychic events, perceived by the patient or an observer" [1, 2]. The diagnosis epilepsy is given when patients experience recurrent (two or more) epileptic seizures, unprovoked by any immediate identified cause. The rime between these recurrent seizures has to be at least 24 hours. Multiple seizures occurring within a 24 hour period are considered a single event [1,3]. An epleptic syndrome 4 "an epileptic disorder characterized by a cluster of signs and symptoms customarly occurring together; these include such items as type of seizures, etiology, anatomy, precipitating factors, age of onset, severity, chronicity, diumal and circadian cycling, and sometmes prognosis" [4].

The annual incidence of epilepsy varies from $11 / 100.000$ to $134 / 100.000$ worldwide. The prevalence shows a vatiation ranging from $1.5 / 1000$ to $31 / 1000$ [5]. The variation in the incidence and prevalence rates is mainly due to methodological differences e.g. lack of standardized deftitions, differences in methods of case ascertainment, diagnostic accuracy and seizure classification used in the epidemiological studies $[1,6]$.

In the Netherlands one incidence study has been performed in the area of Maastricht. In this study an incidence of $30 / 100.000$ per year was found for epilepsy [7]. A prevalence of epilepsy of $5 / 1000$ is assumed for the Netherlands [8]. One study found a prevalence of $4 / 1000$ for patients with epilepsy in general practices in the Netherlands [9].

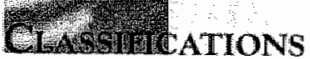

\section{Classifications in general}

A rerminological system relates concepts among themselves in a particular domain. Sometimes these concepts are accompanied with definitions and codes [10]. The concepts in terminological systems are ordered in hierarchical or non-hierarchical relations.

In a hierarchical terminological system relations are characterized by 'is-a" or "part-of" relations. A classification is a hierarchical terminological system. A classificarion is defined as 'an ordered system of concepts within a domain with implict or explicit ordering principles". In a classification a concepe bears information on the level of detail of the related concept and on the hierarchical relation with its parent concept 111. Concepts are arranged based on their essential characteristics into groups of concepts, called classes [10]. An example of a classification is the International Classification of Diseases (ICD) which is a classification of diagnoses with a hierarchical ordering [12].

In non-hierarchical teminological systems relations are used for composition rules for composing (new) complex concepts [10]. SNOMED CT (Systematized Nomenclature of Medicine - Clinical Terms) is an example of a terminological system which consists of 18 modules e.g finding, disease, social context. Within one module concepts are hierarchically related to each other. By linking concepts of the various modules one can compose new medical concepts indicating non-hierarchical telations [13].

A terminological system based on diagnostic categories is introduced for many purposes. 
It brings uniformity into the use of diagnostic terms for purposes of communication and comparison of cases. Secondly an accurate terminological system can support choosing the most appropriate therapy and evaluating different therapies. Also for the complete and accurate registration of medical statistics (incidence/prevalence) it is essential that diseases are classified according to a uniform terminological system $[14,15]$.

\section{Classifications for epileptic seizures}

Many terminological systems for epileptic seizures have been developed. Already in the year $175 \mathrm{AD}$ Galen differentiated between seizures of cerebral (idiopathic) and extracerebral (symptomatic) origin. Throughout the Middle Ages this remained the prevailing doctrine. In the early $18^{\text {th }}$ century other classifications were introduced. Tissot differentiated between petit (minor) and grand (major) mals or seizures in 1772. Calmeil distinguished absences, petit (minor seizures other than absences) and grand mals [15-17].

In the $20^{\text {th }}$ century the development of electroencephalographic (EEG) monitoring became an important instrument to identify foci of epileptic seizures. This led to new discussions about terminological systems for seizures resulting in several (proposed) classification schemes from 1964 onwards.

Note that the classification of epileptic seizures must be distinguished from the classification of epilepsies and epileptic syndromes since a seizure type is one aspect in the diagnosis of an epileptic syndrome. In this thesis we focus on the classification of epileptic seizures.

\section{Clinical and Electroencephalographical Classification of Epileptic Seizures (1969)}

In the 1960 s members of several European branches of the International League Against Epilepsy (ILAE) felt the need for a standardized and uniform international classification for epileptic seizures, because existing classifications varied considerably. In 1964 they presented a preliminary classification [18]. Discussions on this preliminary classification resulted in a revised scheme, although no complete agreement was reached, which was published in its definitive form in 1970 [14, 19].

The ILAE members were fully aware that the classification would be tentative and subject to change in the future due to increased insight into the pathogenesis of epilepsy and advances in the scientific understanding of epilepsy. The ILAE recommended the use of the classification to bring uniformity into the use of diagnostic terms and hence to facilitate communication and comparison of cases, to improve methods for evaluating therapy and eventually to further the understanding of the causes of epileptic seizures [14, 19].

The classification is hierarchically ordered, with epileptic seizures separated into four main categories: partial, generalized, unilateral and unclassified seizures. The categories partial and generalized seizures are subdivided into more specific subtypes (Table 1). In the 1969 classification each clinical seizure type is described by ictal EEG expressions, interictal EEG expressions, anatomical substrate, etiology and age.

\section{Proposal for Revised Clinical and Electroencephalographical Classification of Epileptic Seizures (1981)}

Since the 1969 classification a new method for studying epileptic seizures was introduced. This method included the possibility to make videotapes containing ellectroencephalographic information and clinical manifestations of epileptic seizures recorded simultaneously. The 
1. Partial seizures (seizures beginning locally)

A. Partal serzures with elementary symptomatology (generally without impairment of consciousness)

1. With motor symptoms (includes Jacksonian seizures)

2. Wuth special sensory of somatosensory symptoms

3. With autonomic symptoms

4. Compound forms

B. Partiall seizures with complex symptomatology (generally with impaiment of consciousness) (terporal lobe or psyctionotor seizures)

1. With imparment of consciousness only

2. With cognilive symptomatology

3. Wilth affective symptomatology

4. With "psychosensory" symptomatology

5. With "psychomitor" symptomatology (automatisms)

6. Compound forms

C. Partial seizures secondarily generalized

11. Generalized seizures (bilaterally symmetrical and without local onset)
A. Absences (petit mal)
B. Bilateral massive epileptic myoclonus:
C. Infantile spasms
D. Clonic seizures
E. Tonic seizures
F. Tonic-clonic seizures (grand mal)
G. Atonic seizures.
H. Akinetic seizures

11. Unilateral seizures (or predominamitly unillateral seizures)

IV Unclassified epileptic seizures (due to incomplete data)

Tatble 1. Clinical and Ezlectroncephalographical Cassificarion of Epilepric Seizures 1969 [14]

joint andysis of behaviour and EEG pattems allowed a new refined assessment of parienrs. To accommodate these new insights a proposal for a Revised Clinical and Electroencephalographical Classification of Epileptic Seizures (ICES) was presented in 1981 [20]. As stated in the premble this propusil represented a compromise of views of many experts.

The 1981 classification is a clinically applicable classification of epileptic seizures, which may also be whithle to researchers even though the revised classification did not differ much in structure from the 1969 classification [21]. The most important changes are listed below:

1. The criteria for seizure classification are limited to empirical data: the observable clinical manifestations with its accompanying EEG-data. The anatomical substrate, etiology and age are not included anymore. 
2. Subdivision of the partial serzures into subclasses based on preservation of consciousness (responsiveness). Consciousness is the abiliry to respond to exogenous stimuli and can be tested by alsking questions to the patient during the seizure or by evaluating reaction times. The evaluation of responsiveness is entirely empirical and consistent with the narure of the seizure classification.

3. The progression of a partial into a generalized seizure is now explicitly present as a separate seizure type.

4. There is an overlap in the characteristics for symproms of simple parcial seizures and complex partial seizures except for automatisms which are unique to complex partial seizures.

1 Partial seizures (seizures beginning locally)

A. Simple partial seizures (consciousness not impaired)

1. With motor symptorns

2. With somatosensory or special sensory symptoms

3. With autonomic symptoms

4. With psychic symptoms

B. Complex partial seizures (with impairment of consciousness)

1. Beginning as simple partial seizures and progressing to imparment of consciousness

a. With no other features

b. With reatures as in A: 1-4

c. With automatisns

2. Beginning as simple partial seizures. and progressing to impaiment of consciousness

a. With no other features

b. With leatures as in A. 1-4

c. With automatisms

c. Partial seizures secondarily generalized

1. Simple partial seizures evolving to generalized seizures

2. Complex partial seizures evolving to generalized seizures:

3. Simple partial seizures evolving to complex partial seizunes evolving to generalized seizuses

11 Generalized seizures (bilaterally symmetrical and without local onset)

A. I. Absence seizures:

2. Atypical absence seizures.

B. Myocionic seizures

C. Clonic seizures

D. Tonic seizures

E. Tonic-clonic seizures

f. Atonic seizures

III Unilateral seizures (inadequate or incomplete data)

IV Unclassified eplleptic seizures (due to incomplete data)

Table 2. Proposal for Rewised Clinical and Electroncephadographican Classification of Epileptic Seizures 1981 |20| 
5. Elimination of the groups entitled "compound forms". This term was never used by climicians.

6. Elimination of "akinetic seizures" as a distinct seizure. Akinetic implies "without morion" which occurs in a number of seizure types and is nor unique to any specific seizure type.

7. Removal of "infantile spasms" from the classification since it is an age-related syndrome and not a specific seizure type $[16,21]$.

This 1981 classification is bierarchically ordered, with epileptic seizures separated into three main categories: partial, generalized, and unclassified seizures. The categories partial and generalized seizures are subdivided into more specific subtypes (Table 2). For each seizure type the following items are described in the classification: clinical seizure type, ictal EEG expressions and interictal EEG expressions [20].

Despite the fact that the ICES of 1981 proved to be useful in the clinic and an effective tool for communication between scientists, criticism on this classification exists.

First the separation of simple partial seizures and complex partial seizures on the basis of whether or not consciousness is impaired is criticized. Some physicians interpret 'complex' as 'an involvement of higher cortical function' and such an interruption of higher cortical processes cannot be measured by resting the patient's responsiveness. Loss of responsiveness (but retained awareness) resulting from e.g paralysis is measurable but not considered sufficient to classify the seizure as complex partial according to this group of physicians.

The separation of simple partial seizures from complex partial seizures is also criticized because some physicians are reticent about classifying 'psychic symptoms' as simple partial seizures. According to them 'psychic symptoms' are complicated events which require highly integrated cerebral functions so the event is a priori a complex partial seizure [22].

Another point of criticism is the lack of a category for generalized seizures that progress over time e.g. absence seizures that progress into generalized tonic-clonic seizures [22].

Fourth the ICES is not very useful for evaluating epileptic patients for surgery. Seizure manifestations give important information about the localization of the seizure in the brain. The ICES of 1981 does not sufficiently describe the symptomatology of the partial seizures to characterize and localize the onset of these seizures [23, 24].

Finally the ICES of 1981 takes into account the EEG-findings which means that a seizure can be classified accurately only if the EEG is available. However, in a large number of patients an EEO is not available. In most cases detailed EEO-findings are necessary to identify if the seizure was generalized or partial or if the consciousness was altered or lost so that the distinction between a simple and a complex partial seizure can be made [23, 25]. Moreover, ictal EEG-registration is difficult since it requires reconding during the seizure. In many cases only inter-ictal EEGs are available.

\section{Semiological Seizure Classification (1993)}

The criticism on the ICES of 1981 resulted in the idea that there is a need for a seizure classification based exclusively on clinical ictal manifestations, called the semiological seizure classification (SSC) [25].

The SSC (Table 3) is based on ictal seizure semiology reported by patients/observers or as analyzed during video monitoring. The used terminology describing the seizures clarifies the difference between the seizure and epileptic syndrome classification (e.g. a dialeptic 
Aura
a. Somato-sensory
e. Olfactory
b. Visual
1. Autonomic
c: Auditory
g. Abdominal
d. Gustatory
h. Psychic

Autonomic seizure

Dialeptic seizure

Motor seizure

Simple motor seizure
a. Clonic
d. Epileptic spasm
b. Tonic
e. Myodonic
c. Tonic donic
1. Versive

\section{Complex motor seizures}
a. Automotar
c. Gelastic
b. Hypermotor

Special seizures
a. Aphasic
d. Akinetic
b. Astatic
e. Hypomator
c. Atonic
f. Negative myoclonic

Table 3. Semiological Seizure Classificanion [26]

seizure - isolated alteration of awareness - does not tell us whether the patient has in partical or generalized epileptic syndrome). The SSC can be used for diagnosing patients of any ange. The SSC classifies seizures in four groups: auras, autonomic seizures, dialeptic seizures, motor seizures. When seizures cannot be classified in one of the mentioned groups they are categorized as "special seizures' (seizure with negative manifestations). Additional information e.g. EEG-findings should be analyzed separately [26].

The SSC is used successfully for many years at the Cleveland Clinic. Criticism on the SSC is that a) the SSC is a descriptive teminology for seizure manifestations rather than a classification system, b) several important descriptive features are missing which might have usefuld diagnostic and therapeutic implications for the clinician/neurologist in general practice eg. duration and post-ictal behaviour and c) for epilepsy surgery the SSC is useful, but for the clinician/neurologist in general practice the SSC is too detailed $[24]$.

\section{ILAE: Proposal 2001}

In 1997 the ILAE task force on classification and terminology reviewed the most recent ILAE seizure and epilepsy syndrome classifications. They concluded that an update of the 
current classifications to accommodate current insights would not be possible and proposed a new diagnostic scheme that makes use of standard terminology and concepts to describe individual patients. The ILAE suggests that the seizures and epilepsy syndromes have to be described and categorized according to a system that uses standardized terminology and that is sufficiently flexible to take into account that a) some patients cannot be given a known syndromic diagnosis, b) seizure cypes and syndromes change over time when new information becomes available, c) complete and detailed descriptions of ictal phenomenology are necessary but in some cases less detailed descriptions are sufficient for classification and d) multiple classification schemes should be designed for specific purposes e.g. communication and teaching, epidemiological studies and selection of surgery candidates [27].

The proposed diagnostic scheme consists of five axes (Table 4) and is organized to facilitate a clinical approach to determine a diagnosis and therapy in individual patients $[27$, 28]. Axis 1 is a Glossary of Descriptive Ictal Terminology which can be used uniformly to describe ictal events with any degree of detail needed. With axis 2 the seizure type can be determined (from the List of Epileptic Seizures). Axis 3 is the syndromic diagnosis from the List of Epilepsy Syndromes. In axis 4 the etiology - when known-can be specified from a Classification of Diseases frequently associated with epileptic seizures or epileptic syndromes. With axis 5 the degree of impairment (from the WHO ICIDH-2) due to the epileptic condition can be designated [27].

Axis 13 Glossary of Descriptive lotal Terminology
Axis 2
Axis 3
Axis 4 List of Epileptic Seizunes
Axis 5 Classiflcation of Diseases

Table 4. Proposed diagnostic scheme for people with epileptic seizures and with epilepsy [27]

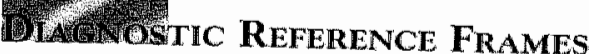

The ICES of 1981 is accepted by epileptologists around the world because of the simplicity of the seizure classification (distinction between partial and generalized seizures and further subdivision), the correlation to anti-epileptic drug (AED) treatment and the relation to the quality of life (e.g. simple partial seizures have a smaller impact on the quality of life than
complex partial seizures) [23].

The ICES of 1981 provides descriptions of the seizure types, which led to variability in its use $[6,29-31]$. For example, a study in which children with epileptic seizures were classified according to the ICES, reported overall kappa values between pairs of observers varying between 0.24 and 0.37 [29]. Another study, including patients aged 15 years or older, found a group kappa value of 0.58 when three observers based their diagnosis solely on clinical judgement. The group kappa improved to 0.73 when the diagnosis was based on diagnostic criteria. This study showed that, when well defined and clearly agreed upon definitions of seizures are used the inter-observer variability decreases [30]. In general kappa values less than 
To compare whether the kappa values found in these studies are high or low we searched, not systematically, for studies reporting about inter-participant agreenent and classification in other medical domains. Fair agreement (kappa of 0.39) among physicians for the classification of arrhythmias in three categories (subjectively interpreted from waveforms) was found. Quantitative measures of the arrhythmias waveform could augment the current standard of subjective classification of arrhythmias [33]. In another study qualitarive categorization of coronary angiograms by two independent observers resulted in a kappa of 0.33. This low agreement was probably due to the lack of strict definitions [34]. When an algorithm is used in the electrocardiographic (ECO) diagnosis of acute myocardial infarction patients with left bundle branch block, an excellent inter-observer agreement (kappa 0.81) was found [35]. These studies in cardiology also indicate that the use of definitions or criteria in classifying or diagnosing reduce the inter-observer variability.

For the diagnostic process comprehensive, (semi-) quantitative and structured descriptions of all seizure types could be helpful. Our aim was to develop such descriptions and called Diagnostic Reference Frames (DRFs) by us.

A DRF is a structured description of a specific seizure type. A frame consists of a list of signs and symptoms (manifestations). For each manifestation a probability is given between 0 and 1. A high probability e.g. 0.9 indicates that a manifestation is frequently occurring. A low probability e.g. 0.05 indicates that a manifestation is seldom occurring in the seizure type. The list of manifestations is identical for all seizure types.

These DRFs can serve as a frame of reference in the process of diagnosing patients with epileptic seizures. There are several clinical and scientific advantages in using DRFs.

A DRF represents the manifestations of a specific seizure type and can serve as a guideline for acquisition and documentation of patient data. Based on the manifestations specified in the DRFs, forms may be designed for a structured approach for collection of patient data. This may increase completeness and uniformity of patient information $[31,36]$ needed for correctly classifying seizures. The DRFs can also be used as an auditing tool by the physician to check whether the patient's manifestations are in agreement with the manifestations listed in the DRF for the seizure type that he/she (tentatively) diagnosed.

From a scientific point of view the univocal use of terminology for recording patient data and classifying seizure types uniformly enhances scientific exchange and discussion about optimal treatment strategies. Also incidence and prevalence rates of seizure types can be compared between regions because the seizure types are classified based on the same terminology and criteria.

We searched for structured seizure descriptions in published resources. The descriptions of the signs and symptoms of different seizure types are scattered over various pulblications that quite often use a different terminology $[20,37-45]$. Sonetimes qualitative terms (e.g. usually, uncommon) are used to indicate the frecuency of occurrence of signs and symptoms and sometimes quantitative information is given. Because in each qualitative study authors used their own set of concepts to describe the seizure types and because in the quantitative studies different study popularions were included on which frequency estimates about the occurrence of signs and symptoms were based, a meta-analysis approach is not feasible.

To derive the DRFs an observational study can be performed. For such a study a lot of patient cases are needed. For each case the seizure manifestations must be reported completely and the diagnosis of the seizure type must be given. Based on this information one can quanrify how often each manifestation occurs in a specific seizure type. Another 
method to develop the DRFs is to perform a knowledge acquisition study among neurologists cpileptologists and ask them to estimate the frequency of occurrence of manifestations in each seizure type. In both methods the physician is crucial: the quality of observational sudy depends on the accuracy of the classification provided by the physician and the quality of the knowledge elicitarion study depends on the accuracy of the estimates provided by the neurologists/epileptologists.

DRFs derived from experts" knowledge could serve as a starting point for an observational study in which the accuracy of the frequency of occurrence of manifestations is refined.

\subsection{WTH OF THE THESIS}

The aim of our research is to develop valid Diagnostic Reference Frames for epileptic seizures. We decided to base the DRFs on the knowledge and expertise of experts. This development consists of three stages: a) the dewelopment of a methodology to elicit knowledge among experts b) the knowledge elicitation itself and c) the validation of the knowledge included in the DRFis.

\section{Methodology}

In a pilot study we test whether it is feasible to obtain Diagnostic Reference Frames for seizures by querying neurologists/epileptologists and deriving knowledge from their opinions. Experts are asked to select most frequently occurring seizure manifestations for each seizure type on an electronic form. In chapter 2 the inter-participant agreement in the selection of seizure manifestations for the description of the seizure types is evaluated. Chapter 3 describes whether the seizure descriptions given by the experts are discriminative.

Further we present a general approach to identify experts who provide largely deviating opinions in a knowledge acquisition procedure. With this approach it is possible to assess whether neurologists/epileptologists involved in the knowledge acquisition process provide reliable data. The reliability of the responses of the neurologists/epileptologists is calculated with the intraclass correlation coefficient (ICC). This is described in chapter 4.

\section{Knowledge elicitation}

Chapter 5 reponts on the knowledge elicitation procedure. The recruitment of the neurologists/ epilleptologists is described in detail. The experts provided an estimate of the frequency of occurrence for each manifestation listed on the redesigned electronic form (redesign based on the evaluation of the design of the pilot study). For each manifestation the mean estimated frequency of occurence is computed from the frequency estimates of the experts for each seizure type.

\section{Validation of DRFs}

The validity of the DRFs for clinical use is evaluated and described in chapter 6 . Patient's manifestations are entered in a decision support system and by successively applying Bayes's rule posterior probabilities are calculated. The DRFs with the highest posterior probability gives an indication of the classifcation of the seizure. In this way we can assess the accuracy of the Diagnostic Reference Frames in classifying patients with epileptic seizures.

We also used the DRFs in an educational setring. We assessed how complete and 
accurate medical students and neurology residents are in reporting seiarure manifestations since a good classification of seizures is based on accurace observing and reporting manifestations. The seizure classification provided by the medical students and neurology residents is compared to the classification given by Bayes's rule based on the reported seizure manifestations by the participants. This is described in chapter 7.

Chapter 8 presents the conclusions and discusses general aspects of the findings as well as a methodological reflection and recommendations for practice.

The chapters 2.7 in this thesis are based on manuscripts published in, submitted for or in preparation for publication to scientific journals. The manuscripts were written with the intention to be read independently. This inevitably resulted in some overlap between the chapters. 
Chapter 1 
1. Commision on Epidemiology and Prognosis of the Intematond League Against Bphepsy. Cwathin for epidemiologic studies on epilepsy. Epalepsia $1993,34(4), 592 \cdot 6$.

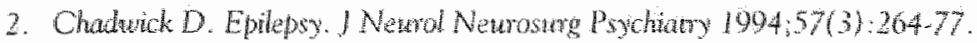

3. Commision report of the Intemational Lague Aganst Eplepsy. The epidemology of the aptaphis: future drections. Epilepsia 1997;3815):614.8.

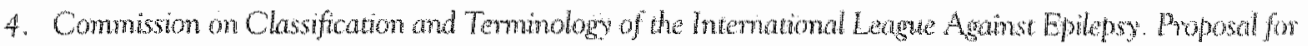

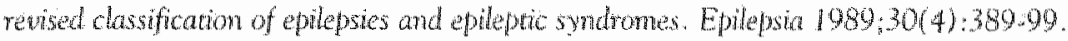

5. Cokerell OC, Shomon SD. Epilepsy: cument conceps. London: Cwmen Medical Literawe; 1996 .

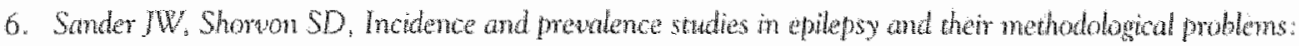
a revien. I Neurol Neurosurg Psychatry 1987,50(7):829-39.

7. Kotsopoulos IAW, de Krom MCTFM, Kesstes FGH, Lodder J. Troost I, Twellaw M, win Murde T.

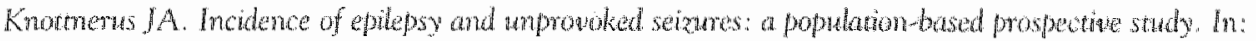

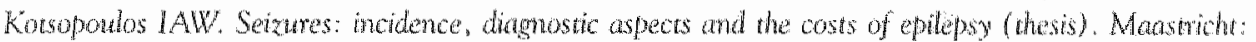
Unigraphic Uniqersineit Masasticht; 2002:39-51.

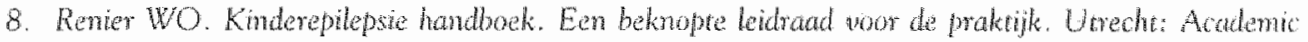
Phamacettical Productions; 2003.

9. Rurgers MJ. Ephepsy in general practice: the Durch situation. Epritepsia 1986:27(6):734-8.

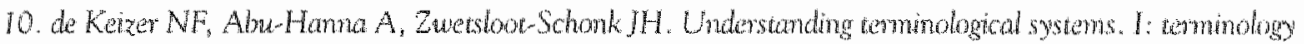
and typology. Methods inf Med 2000,39(1):16-21.

11. qan Bemmel J, Musen M. Hanbook of medical informatics. Houten/Diegem: Bohn Stallun Van Loghum: 1997.

12. World Heath Orgomization. Intemational Classification of Discases: 2004 : wat who wh:

13. SNOMED Intemational. What is SNOMED CT?; 2004: whesnomed.ong.

14. Gastan $\mathrm{H}$. Clinical and electroencepholographical clasification of epplepric serwes. Epilepsia $1970,11(1): 102,13$

15. Dreifuss FE. Classification of epileptic seizures. In: Engel J Ir, Pedly TA, editors. Epilapsy: a comprehensive textbook. New York: Liphincoti-Ranch; 1997:517.24.

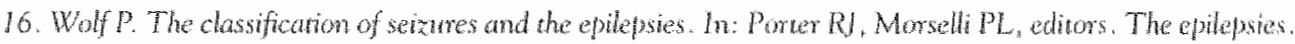
Commall: Butterworth 60 : 1985:106-24.

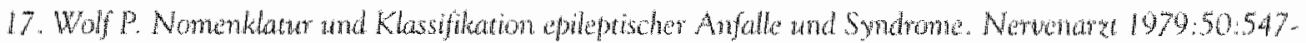
54.

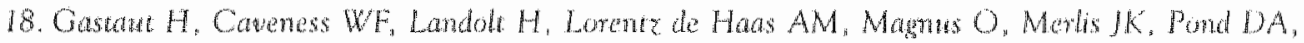

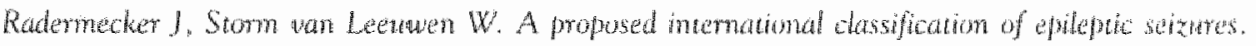
Epildosia 1964:5:297-306.

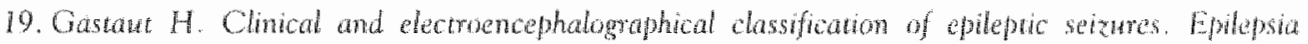
1969, $10(5$ ppl $: S 2-53$.

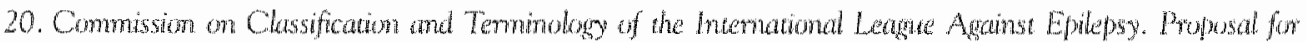

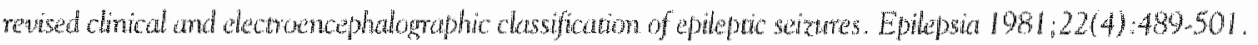

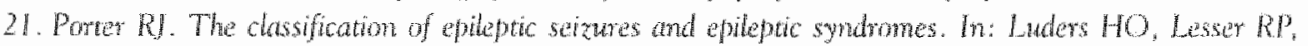
dions. Epilepsy doctronchical syndromes. London: Springer; 1987:1.11.

22. Porter RI. Recognizing and classifyng eplepric seizures and epilepto syndromes. Newrol Chin $1986: 4(3): 495-508$.

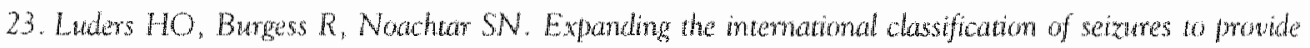
localiation information. Neurology 1993:4369):16505. 


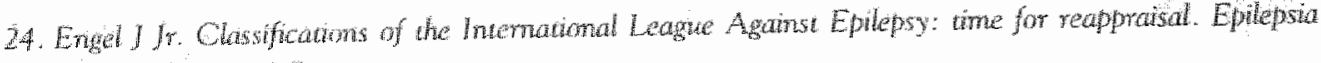
$1998,39(9): 10147$

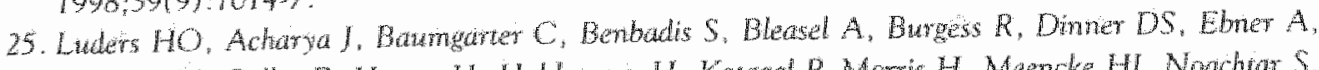

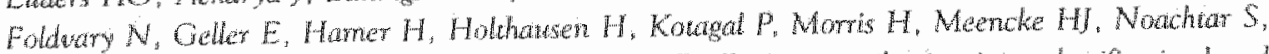

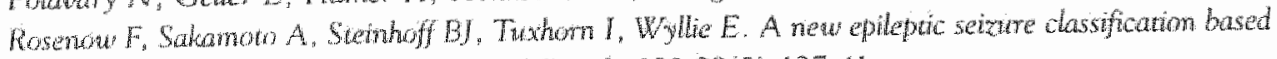
Exchwively on ictat semilogy. Acta Newol Scand 1999,99(3):137-41.

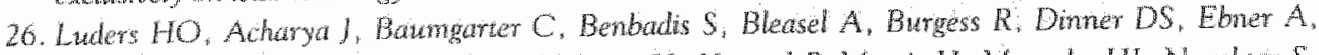
Folvary N, Geller E. Hamer H, Hohthasen H, Kotagal P, Morris H. Meencke H. Nachar S.

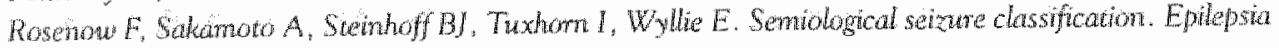
$1998 \cdot 39(9): 1006 \cdot 13$

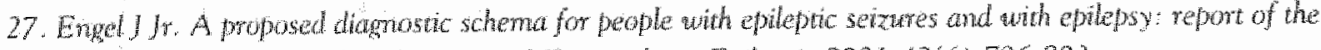
ILAE Task Force on Clasification and Temninology. Epilepsia 2001:42(6):796-803.

28. Wolf P. Focal seivures and eplepsies. Ephlepsia 2002:43(Suppl 8):30.

29. Bodenstemer JB. Brownsumth RD, Knapik JR, Kanter MC. Cowan LD, Lew in A. Interobserver

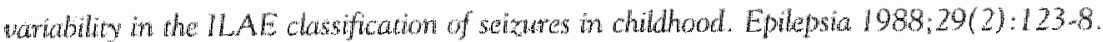

30. man Donsetarar CA. Ceers AT, Meulsted, Habbema JD. Siad A. Reliability of the diagnosis of a first seizute. Neurology 1089;39(2 Pr 1):267-7).

31. Rewtun DC, Howel RA, Gebar KE, Berkonic SF. Valdation of a questionname for dinical seizare diagnosis. Epilapsia 1992:33(6):1065-71.

32. Landis IR. Koch $\mathrm{GO}$, The measmement of observer agrement for categonical data. Biometrics $1977,33(1): 159-74$

33. Ligh foor CB, Sorensen Th. Garfinkel MD, Sheman LD, Calkway CW Menegazi IJ. Physichan

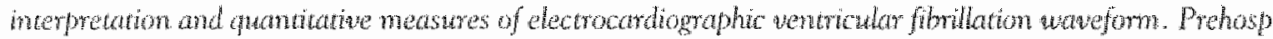
Energ Care 2001: 5(2):147-54.

34. Hemmo JP. Azat A, Umans VA, Boersma E, non Es GA, Serruys PW. Inter-and intro-obserwer

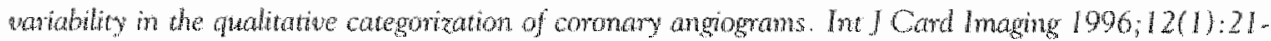
30.

35. Sokolowe PE, Syarbossa EB, Amsterdam EA, Gelber R, Lee TC, Maynard, C, Richards JR, Valonte R. Wagne $\mathrm{OS}$. Interobsewer agreement in the electrocardographic diagnosis of acme myocardial infarcron in patients with left bundle branch block. Ant Emerg Med 2000;36(6):566-71.

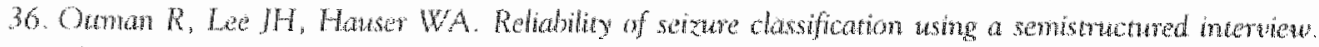
Newolugy $1993 ; 43(12): 2526-30$.

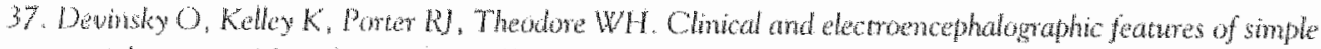

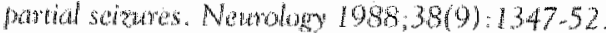

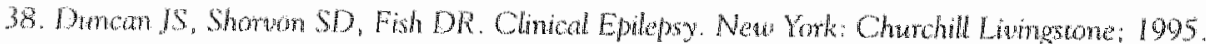

39. Gram $L$. Eprtepric seizumes and syndromes. The Lancet 1990:336(8708):161-3.

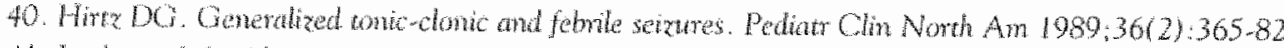

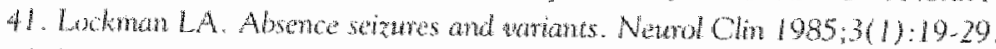

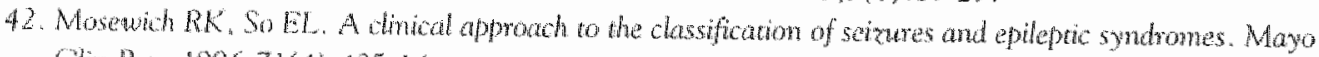
Gin Pro $1996 ; 71(4) \times 405-14$.

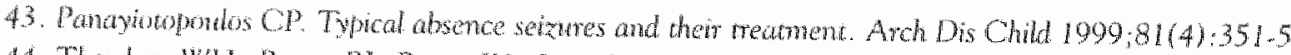

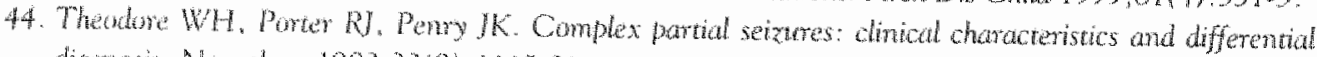
diugnosis. Newolog 1983,33(9):1115.21.

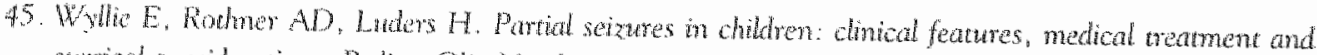
surghal considerations. Pediat Clin Nonth Am 1989:36(2):343-64 



\section{Chapter 2}

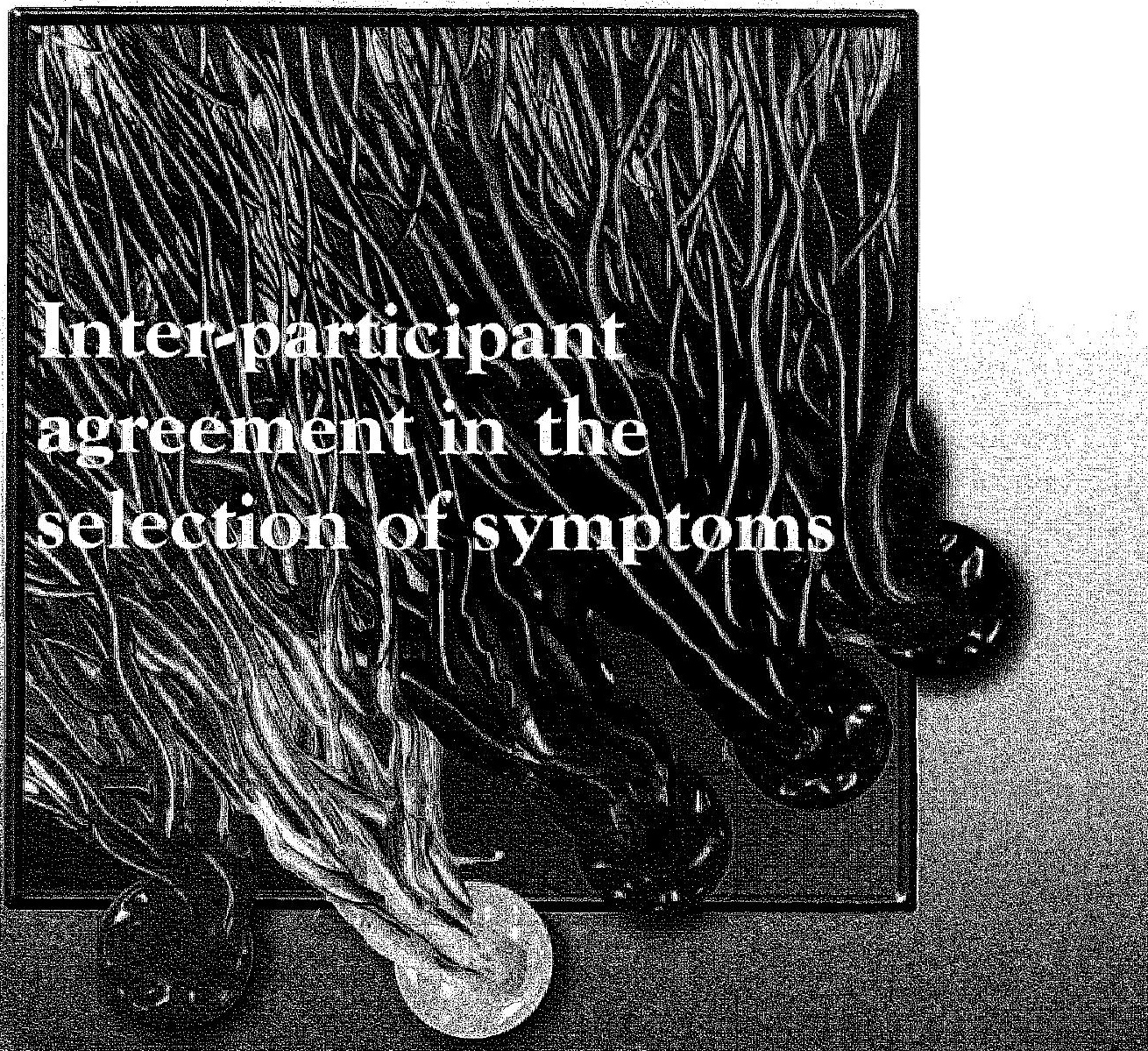

Published as:

vin Ast IE Talmen JL, Renier WO, Ahles PPM, Hasman A.

Development of diagnostic reference frames for seizures.

Part 1 inter paricipant agreement in the seleetion of symproms.

Internationd Joumal of Medical Informitics 2003,70(2-3),285-292. 


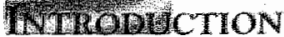

An epileptic seizure is a clinical manifestation characterized by an intermittent and stereotyped disturbance of consciousness, behavior, emotion, motor function or sensation caused by an abnomal and excessive discharge of a set of neurons in the brain. Epileptic seizures vary with respect to their clinical features, etiology, severity and prognosis $[1,2]$.

In the ninetern-sixties, members of several European branches of the International League Against Epilepsy (ILAE) felt the need for a standardized and uniform classification system for epileptic seizures. In 1964 they presented a preliminary classification [3]. This classification was published in its definitive form in 1969. The ILAE members were fully aware that the classification would be subject to change in the future due to increased insight into the pathogenesis of epilepsy. A revised International Classification of Epileptic Seizures (ICES) was proposed in 1981 [4]. This classification is hierarchically ordered, with epileptic seizures separated into partial, generalized and unclassified categories. The two classifiable groups of seizures are further subdivided into more specific subrypes (Table 1).

$1,+$ Partial seizunes (PS)
A. Sinple partial seizures (SPS)
B Complex partil seizures (CPS)
C Partial seizures secondary generalized (SCS)

$14+$ Pimary Generalized selzures (PGS)
A. Absence seizures (AS)
1 Tyical absence seizures (TAS)
2. Atypical absence seizures (AAS)
B. Moclonic seizures (MS)
C Clonic seizures (CS)
D. Tonlc selzures (TS)
E Toniceonic seizures (TCS)
5 Atonic serrures (ATS)

$11, \quad$ Unclassified epileptic seizures (US)

Table 11. International Classification of Epileptic Seiaures [4]

The ILAE recommends the use of the classification to bring uniformity into the use of diagnostic terms and hence to facilitate communication on seizures and comparison of cases, to improve methods for evaluating therapy and eventually to further the understanding of the causes of epileptic seizures [5]. To reach these goals, the concepts used in the classification should be clearly defined. However the classification gives only a rather limited description of the seizure types, which leads to variability in its use. This has become apparent in several studies [6-9]. For example, a study in which children with epileptic seizures were classified according to the ICES, reported overall kappa values between pairs of observers varying 
between 0.24 and 0.37 [6]. Another study, including patients aged 15 years or older, found a group kappa value of 0.58 when three observers based their diagnosis solely on clinical judgement. The group kappa value improved to 0.73 when the diagnosis was based on diagnostic criteria. In this study the diagnostic criteria consisted of six definitions of seizures from which the observers had to choose when classifying patients [7]. This study showed that the use of (generally accepted) definitions of seizures may decrease the inter-observer variability.

We suggest to develop structured descriptions of signs and symptoms of specific seizure types (called Diagnostic Reference Frames -DRFs- by us) which can serve as a frame of reference in various situations. First of all, observed patient's signs and symptoms can be matched against the DRFs and the DRF with the highest match provides an indication for the seizure type present. The DRFs may also be used to generate lists of questions about the presence or absence of symptoms that potentially discriminate between competing diagnoses based on information gathered so far during the consultation. This results in a checklist and may increase completeness in the registration of patient's symptoms in the (electronic) patient record $[8,10]$. Finally, the DRFs may be used as an auditing tool for the physician to check whether the patient's symptoms are in agreement with the symptoms listed in the DRF for the seizure type that he/she tentatively diagnosed.

Published reports, however, do not provide comprehensive descriptions of seizure types. In artificial intelligence it is customary to elicit knowledge from experts; we adopted a similar approach in our project. To that end we developed an electronic form on which neurologists/epileptologists could identify symptoms they consider as frequently occurring (present in at least $75 \%$ of the cases) in each of the different seizure types. The assumption is that by querying experts reliable descriptions of the various seizure types from the opinions of neurologists/epileptologists can be constructed.

Via this feasibility study we provide an answer to the following research questions: 1) Can the observed degree of agreement among neurologists be explained by either pure random selection of frequently occurring symptoms or by a core of common, frequently accurring symptoms on which there may be disagreement only in its extent? The latter explanation would provide support for our approach to building DRFs. 2) How many neurologists are needed to derive a reliable set of symptoms for the construction of DRFs?

In our second accompanying paper "Development of Diagnostic Reference Frames for Seizures. Part 2: Are seizure descriptions discriminative?" [11], we determine whether the symptoms considered by the neurologists/epileprologists as frequently occurring in the various seizure types result in seizure descriptions with which seizure types can be distinguished.

First the methodological aspects of this study are described. Next, an overview of some characteristics of the participating neurologists/epileptologists and the results of the analyses are given. The results are discussed in the last section.

\section{MLarions}

\section{Electronic form}

On the electronic form 239 symptoms, relevant in the context of describing seizure types, were listed. Participants were asked to identify frequently occurring symptoms (present in at least $75 \%$ of the cases) and select these symptoms on the electronic form for the most common seizure types. 
Symptoms not considered as frequently occurring (present in less than $75 \%$ of the cases) had to be left unchecked.

Because it would take too much time for a single participant to identify the frequently occurring symptoms for all nine seizure types, we designed four forms each consisting of rwo or three seizure types. Each of the four forms contained the same set of symptoms. The participants could select the form they would like to fill out. More information about the design of the electronic form can be found in chapter 3 [11].

\section{Participants}

Participants were recruited at the $24^{\text {th }}$ International Epilepsy Congress in Buenos Aires in May 2001. The inclusion criterion was that the participant was a practitioner in epilepsy care and details requested were: their profession, number of years of clinical experience, how many epilepsy patients they see per week on average and whether the participant is a member of the ILAE Task Force on Classification and Terminology.

\section{Analysis}

A. Our null hyporhesis was that the number of symptoms on which a certain number of participants agree would not differ significantly from the number found by pure random selection of symptoms. Therefore we compared the observed agreement among the participants with the agreement expected by chance. This method is described by Giuse et. al. who used it to evaluate the inter-author agreement among seven internists who were asked to extract precise diagnostic information for use in Quick Medical Reference (QMR) by reviewing 109 articles on acute perinephric abscess [12].

Our list of symptoms served as a candidate list of terms from which each participant $i$ selected a certain number of symptoms $m_{i} ; i=1 \ldots n$ present in a seizure type. One can determine for each seizure type, from the data entered by the participants, how many participants selected each symptom. By counting how many symptoms are selected by 0 , 1 , $2, \ldots, n$ participants one obtains a profile, which can be compared with the profile derived from a simulation in which each participant i would select $m_{i}$ symptoms randomly. To get a proper estimate of the distribution based on random selection for each seizure type, the simulation was performed one million $\left(10^{6}\right)$ times and the results were averaged. The observed and simulated inter-participant agreements were compared using the $\chi 2$-test with a significance level of $\alpha=0.01$ (after merging adjacent categories with expected frequencies
of $<1)[13]$.

B. In the second analysis we ranked the participants according to the number of symptoms they selected per seizure type in increasing order: $m_{1} \leq m_{2} \leq m_{3} \ldots \leq m_{n}$. The idea is that the participant who selected the lowest number of symproms only identified the most relevant frequently occurring symptoms. Furthermore, we assume that participant $k$ selects the same symptoms as participant $k-1$ plus some additional ones. In this model it is obvious that all participants will agree on $m_{1}$ symptoms, that at least $n-1$ participants agree on $m_{2}$ symptoms etc. So, at least $k$ participants will agree on $m_{n+1-k}$ symptoms. This provides a observed in our study.

C. The reliability coefficient (Cronbach's alpha) was calculated for the judgements of the participants. Systematic differences between observers were assumed to be absent. The reliability coefficient measures the ratio of the variation in the seizure descriptions due to 
symptoms (true variance) to the total variance of the data (true variance and error variance) [14; p. 117]. The true variance is the variability due to the presence or absence of symptoms in a type of seizure. The error variance is due to different opinions of participants concerning the presence or absence of specific symptoms. If the results are to be used for a general description of DRFs, a reliability coefficient of 0.7 is sufficient. A reliability coefficient of $0.90-0.95$ is needed for deciding which symproms to include in a specific DRF $[14,15]$.

To determine the number of participants needed we used the Spearman-Brown prophecy formula [14; p. 99].

\section{SILIS}

\section{Participants}

Twenty-four participants filled out the electronic form. Nineteen participants came from Europe, one from Asia and four from the Americas. Twenty-one participants were neurologist, epileptologist or resident in one of the mentioned professions $(9,9$ and 3 , respectively). One paediatrician, one psychologist and one clinical neurophysiologist filled out the electronic form as well. All participants filled out one form, except one who filled out rwo forms. Fourteen participants had 10 or more years of clinical experience. Sixteen participants treat on average at least one epilepsy patient per day. Two participants were members of the ILAE Task Force on Classification and Terminology.

Table 2 gives a breakdown of the number of participants who selected symptoms for the different seizure types.

\begin{tabular}{|c|c|c|}
\hline Form & Seizure type & Number of participants \\
\hline 1 & Simple partial seizures (SPS) & 9 \\
\hline & Complex pantial seizures (CPS) & 9 \\
\hline 2 & Pinmary geinealized seizures (PCS) & 5 \\
\hline & Secondary Beneralized seizures (SCS) & 5 \\
\hline 3 & Absence seizures (AS) & 6 \\
\hline & Myodonic seizures (MS) & 6 \\
\hline & Tonic-clanic seizures (TCS) & 6 \\
\hline 4 & Typical absence seizures (TAS) & 5 \\
\hline & Atypical absence seizures (AAS) & 5 \\
\hline
\end{tabular}

Table 2. Number of parricipants who selected symproms for the different seizure types

\section{Analysis}

A. We compared the observed profiles with the profiles obtained from the simulation for each of the selected seizure types. As an example we provide details for complex partial seizures (CPS). Results for the other seizure types are available at appendix I.

The number of selected symptoms for CPS ranged from 12 to 120 (Table 3). Table 4 
gives an overview of the range in the number of selected symptoms by the participants for each seizure type. For arypical absence seizures (AAS) and complex partial seizures (CPS) the largest number of selected symptoms is ten times the lowest number. For simple partial seizures (SPS) there was a factor 8.5 difference between the minimum and the maximum number of selected symptoms by the participants.

\begin{tabular}{|c|c|c|c|c|c|c|c|c|c|}
\hline Participant & Partil & Part.2 & Parti 3 & Part.4 & Part.5 & Part.6 & Part.7 & Part.8 & Part.9 \\
\hline $\begin{array}{l}\text { Number of selected } \\
\text { symptoms }\end{array}$ & 12 & 36 & 40 & 44 & 51 & 66 & 73 & \|\| $\mid$ & 120 \\
\hline
\end{tabular}

Table 3. Number of selected symptoms by each particinant for complex parrial seizutes

$\begin{array}{lccccccccc}\text { Seizure type } & \text { SPS } & \text { CPS } & \text { PGS } & \text { SCS } & \text { AS } & \text { MS } & \text { TCS } & \text { TAS } & \text { AAS } \\ \text { Rainge } & 10-85 & 12-120 & 37-76 & 40-141 & 19-58 & 24-64 & 43-109 & 13-47 & 11-114 .\end{array}$

Table 4. Range in number of selecred symptoms by participants for tach seizure type

Table 5 and Figure 1 present the observed and simulated inter-participant agreement profiles for CPS. The table indicates that 47 symptoms were never selected by the neurologists/epileptologists compared to 13.8 on average during the simulation (agreement level 0). At agreement level 3, 34 symptoms were selected by three neurologists/epileptologists compared to 60.5 on average during the simulation.

The number of times that two, three and four participants selected the same symptoms

\section{of \\ participants \\ selecting \\ the same \\ symptoms}

\section{Observed}

number

of sympt.

$\begin{array}{lll}47 & 52 & 45\end{array}$

34

28

16

7

6

$3 \quad 1$

Simulated

number

of sympt.

$13.8 \quad 50.1$

74.8

60.5

29.2

8.8

1.6

0.2

0

Simulated >

Observed*

* Nurnber of times in which simulated participants agreed more than the actual participants

Table 5. Observed and simulated inter-participant agreement profiles for complex parrial seizures 


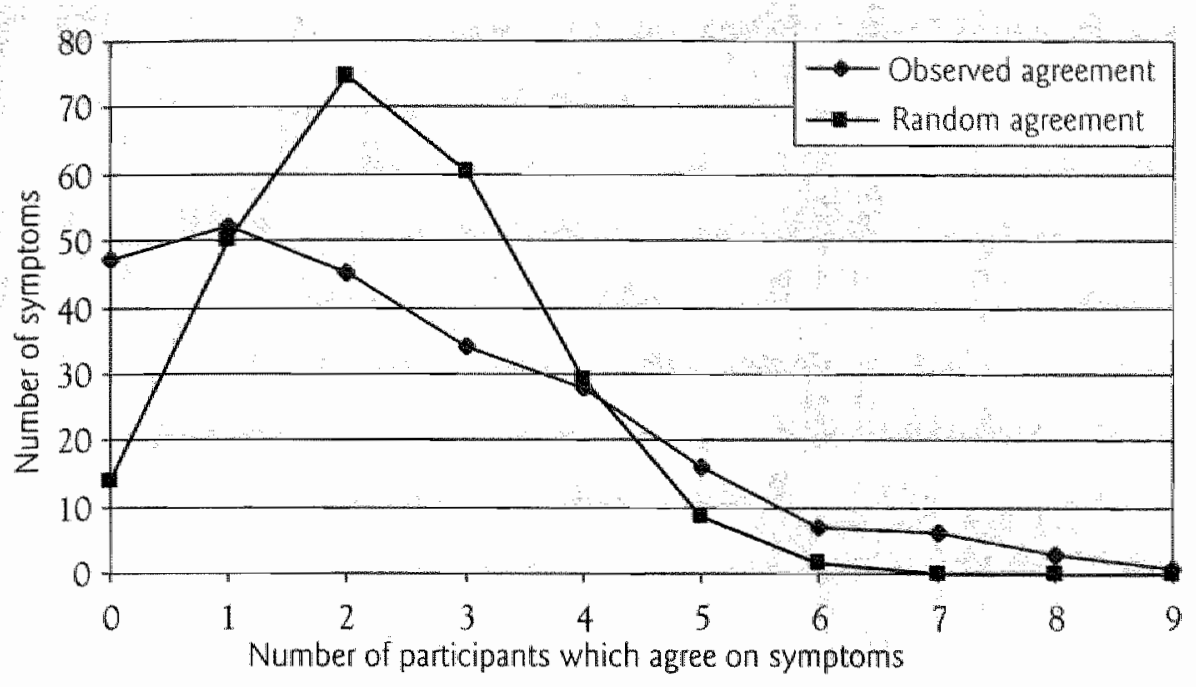

Figure 1. Observed and randon (=simulated) agrement among nine participants for complex partial seizures

for CPS was less than predicted by chance. The number of times that five or more participants selected the same symptoms for CPS was greater than predicted by chance. At: agreement levels seven, eight and nine in none of the one million simulation runs the number of symptoms on which 7,8 or 9 participants, respectively, agreed exceeded the observed number of symproms.

The $\chi^{2}$-test for CPS shows us that the observed distribution of symptoms over the various levels of agreement significantly differs from the simulated distribution $(p<0.005)$. The $\chi 2$-values for the other seizure types also show that the profiles of the number of selected symptoms identified by the participants are significantly different from the profiles predicted by chance (Table 6).

B. For all seizure types the cumulative distribution of the observed agreement is compared with both the cumulative distribution of the maximum agreement artainalale and random agreement. Figure 2 presents the distributions for complex partial seizures. For

$\begin{array}{lccc}\text { Seizure type } & \text { Degrees of freedom } & \text { Critical value } & \chi 2 \\ \text { SPS } & 5 & & \\ \text { CPS } & 6 & 11.070 & 247.0 \\ \text { PGS } & 4 & 12.592 & 236.8 \\ \text { SG5 } & 5 & 9.488 & 189.7 \\ \text { AS } & 4 & 11.070 & 162.6 \\ \text { MS } & 4 & 9.488 & 300.9 \\ \text { TCS } & 5 & 9.488 & 212.9 \\ \text { TAS } & 3 & 11.070 & 308.8 \\ \text { AAS } & 4 & 7.815 & 103.8 \\ & 4 & 9.488 & 95.4\end{array}$

Table 6. $\chi^{2}$-vilues for all included seizure types 
higher levels of agreement, participants agree on less symptoms than what could have been obtained assuming the pure subset model. On the other hand they agree on more symptoms than based on a random model. For the other seizure types similar results are obtained.

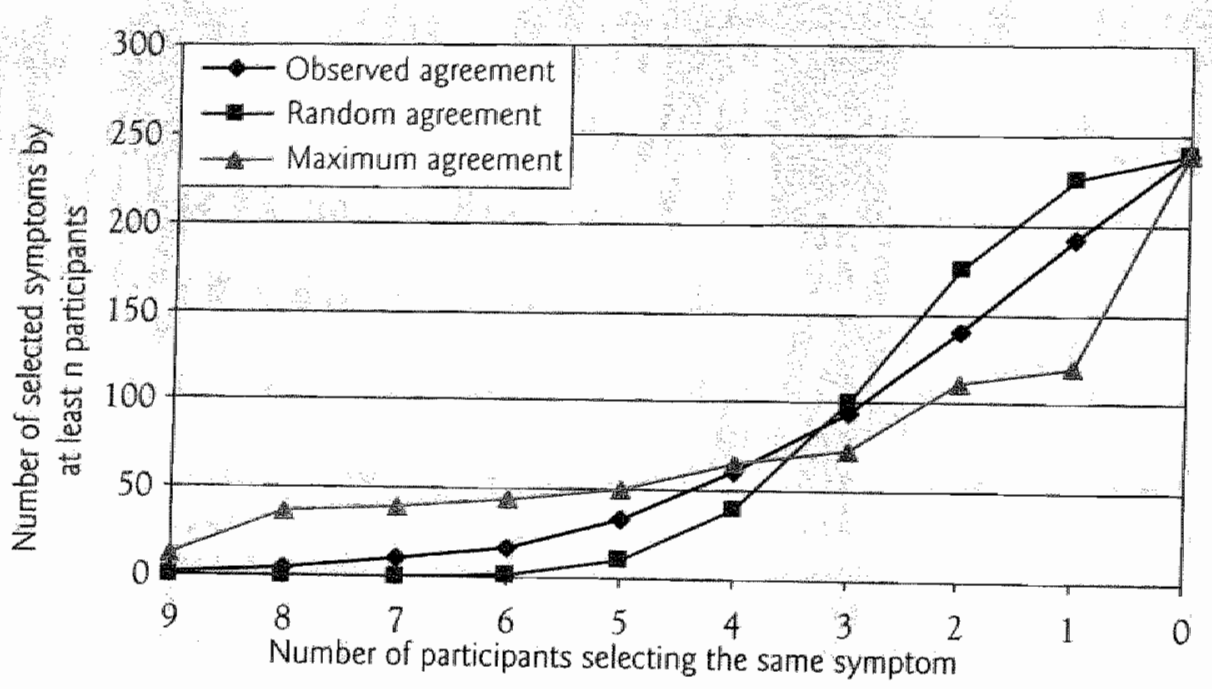

Figure 2. Observed, random and expected maximal agrement for complex parrial seizures

C. The reliability coefficients for the different seizure types are calculated based on the 239 symptoms that could be identified in each seizure type and the number of participants that filled out the electronic form for this seizure type. The reliability coefficients are presented in Table 7. For most seizures, a reliability coefficient around 0.7 (range $0.67-0.74$ ) was found. The reliability was relatively low only for SGS and AAS (0.60 and 0.56, respectively). This means that for SGS and for AAS respectively 40 and 44 percent of the total variance was due to inter-observer variability. The Spearman-Brown prophecy formula showed that in the worst case 81 participants are needed to obtain a reliability coefficient of 0.95 (Table 7) |14; p. 99].

SPS

CPS PGS SGS

AS MS TCS

TAS

AAS

Number of participants

who selected symptoms.

9

9

Cronbach's alpha

$0.70 \quad 0.68 \quad 0.68 \quad 0.60$

0.74

$0.71 \quad 0.74$

0.67

0.56

Number of participants

needed for tho $=0.95$

$\begin{array}{llll}74 & 81 & 44 & 63\end{array}$

63

40

46

40

47

75

Table 7. Retiability coctticients for the in tentified symptons for each seizure type 


\section{Inter-participant agreement}

Our analyses show that the inter participant agreenent for the description of the seizure types significantly differs from chance. It is also clear that the agreement is not optimal since for higher levels of agreement the maximal agreement possible is not reached. Neurologists/ epileptologists that indicated a relatiwely low number of symptoms apparenty do not always indicate symptoms that are seen as highly relevant by the other participants. When we study the majority decisions (i.e. the total number of symptoms on which the majority agrees) the number of selected symptoms is significanty higher than expected by chance and approaches the maximum agreement attainable in all seizure rypes. However, to derive a reliable set of symptoms for the construction of the DRFs more participants are needed.

For complex partial seizures the number of times that agreement at levels two, three and four was observed in the actual profiles was less than predicted by chance, showing a marked shift towards higher agreement among the actual participants for the agrement levels five to nine. Since we found that when the majority of participants selects the same symptoms the observed inter-participant agreement is approaching the maximum agreement atrainable. it is likely that agreement among participants exists on characteristic symptoms for the different seizure types. This is found for all setzure types even though the four forms were filled out by four different groups of participants, with different backgrounds. Apparently there exists at least some consensus on characteristic symptoms for each seizure type. This demonstrates that a group of neurologists/epileptologists working with the same set of symptoms can produce similar resules, in spite of individual differences. Thereby our approach to develop a medical knowledge base of seizure descriptions based on expert knowledge is justified.

Comparable to what we found, Giuse et. al. [12] reported also that agreement among the majority of the participants was significantly higher than predicted by chance. In the study of Giuse et. al. [12] a candidate set of findings, describing acute perinephric abscess, was defined on the basis of 4350 existing QMR findings. A finding was included in the candidate set if one or more participants selected this finding. This candidate set, existing of 296 findings, was used in comparing the actual and simulated agreement among the 7 profilers. In our study we used a list of 239 symptoms, all relevant in the context of describing epileptic seizures. All symptoms were used in comparing the actual and simulated agreement, even if the participants never selected the symptom (agreement at level acro).

The completed disease profiles in the study of Oiuse et. al. [12] ranged in length from 73 to 186 findings (factor 2.5). In our study also a factor of 2.5 to 3 was observed between the minumum and maximum number of selected symptoms. Only for simple partial seizures, complex partial seizures and atypical absence seizures a factor of 8.5-10 was observed. The large factor in these three seizure types may be explaned by their nature: arypical absence seizures are atypical, so it will be difficult to define which symptoms occur frequently. Fon" this seizure type one participant selected a low number of symptoms (11) and one quite many (114). The remaining neurologists/epileptologists chose $66-68$ symptoms. Complex partial seizures have a broad range of symptoms that may occur. Hence it is to be expected that more variarion in the number of selected symptoms is found. Here also we have one participant selecting a low number of syraptoms (12) and two quite many (111 and 120 ). 
In simple partial seizures there seem to be two different groups of neurologists/ epileptologists: one group of six neurologists/epileptologists selecting a relatively low number of symptoms (10-32) and three neurologists/epileptologists selecting a much larger number of symptoms (63-85). Whether a neurologist has to be excluded fron the construction of the DRFs, solely on the basis of the number of symptoms selected, requires further analysis.

Part of the variability in the selection of symptoms for the seizure types may be due to differences in knowledge and experience of the participants. Because of the small number of experts who participated in this study we could not test this hypothesis. Another reason for the variability may be caused by the characteristics of the study. We asked participants to identify symptoms they considered present in more than $75 \%$ of the cases. The threshold of $75 \%$ is probably difficult to assess, resulting in relatively many symptoms that were only selected by one or two participants.

\section{Character of study}

The reliability coefficient for the judgements of the participants for the seizure types varied between 0.54 and 0.74 . To determine how the reliability coefficients obtained in this study relate to the reliability coefficients found in other studies [16-18] we calculated the per rater reliabiliry of the various studies using the Spearman-Brown prophecy formula (Table 8). Compared to the study of Van der Lei et. al. [17], in which eight reviewers were asked to judge 298 comments (made by a decision support system) concerning the care of hypertensive patients as 'correct' or 'incorrect', our reliability coefficients are higher. In the study of Van der Lei et. al. [17] the per water reliability was 0.19 . In our study the per rater reliability varied between 0.19 and 0.32 . On the other hand the reliability coefficients reported in the two other studies we considered are higher $[16,18]$. In one of these studies three reviewers were asked to indicate, based on accepted practice guidelines, whether 1217 requested diagnostic tests were appropriately requested or not (per rater variability of 0.39) [16]. In the study of Hasman et. al. [18] six sets of three pathologists each received a different set of 40 pathology reports, which were all accompanied with 5 different SNOMED diagnostic code lines. Each pathologist was instructed to rate the adequacy of every diagnostic line on a three-point scale. Here the per rater reliability varied between 0.59 and 0.73 . One possible explanation for the differences in the per rater variability in these four studies lies in the certainty with which the question can be answered. In the assessment of appropriateness of treatment decisions in hypertension there could be disagreement on what is appropriate. It could even be that some physicians have considered some of the comments appropriate although the comments might not reflect the best possible comment that could be made. In our study the assessment whether a sympton occurs in more or less than $75 \%$ of the cases may be a cause study of Bindels er. al. the guidelines should have provided the correct answars [19]. In the not consulted, hence the retrievability f have provided the correct answer, but they were Hasman et. al., SNOMED codes represent expressions that normally appear in pathology reports. It is easier to decide whether a code sequence properly describes the contents of a written report and hence a higher per rater variability can be expected.

In our study participants were asked to identify frequently occurring symptoms for each lower per rater reliability coefficients.

According to the assumption that the reliability coefficient should be around 0.90 to 
determine whether a single symptom can be included into a DRF, the reliability coefficients obtained in this study are not sufficient for making reliable seizure descriptions. [15].

To increase the reliability coefficient, more participants must fill out the electronic form. The number of required participants is prohibirively high (Table 7). If we accept a reliability coefficient of 0.90 , we would need 39 participants, which is a more realistic number to include in a study. Using other methods of data collection, e.g. a modified Delphi procedure, could probably reduce this number of required participants. This may reduce obvious errors by the experts and hence increase the reliability coefficient.

In our study the reliability coefficients were determined on the basis of 0.1 scores. This may result in lower values of the reliability coefficients. However it was concluded by Feldt [20] that for a relatively large number of items accurate values can be obtained

\section{Study}

Bindels et, ail, [16]

Van der Lell et al [17]

Hasman et al. [l 8$]$

This study

\section{Per rateir reliability}

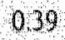

019

0,59073

0.19032

Table 8 . Per rater reliability in differen studies

\section{6ongLISION}

This study shows that although the neurologists/epileptologists do not randomly select frequently occurring symptoms for the different seizure types, the maximum agreement possible is not obtained. However, there exists agreement among the participants on characteristic frequently occurring symptoms for the different seiare types to use expert opinions in the building of reliable descriptions of seizure rypes.

Based on these results we decided to study if the neurologists/epileptologists selected a discriminative ser of symptoms for each seizure type. These results are described in chapter 3 [11]. 


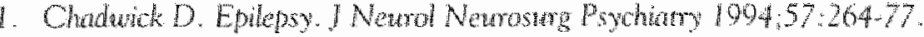

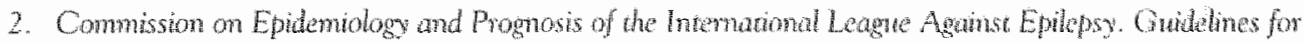
epdeminlogic studies on epilepsy. Epilepsin 1993,34:592-6.

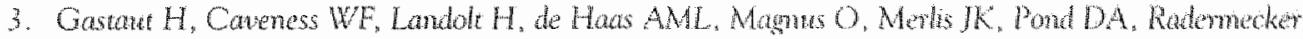

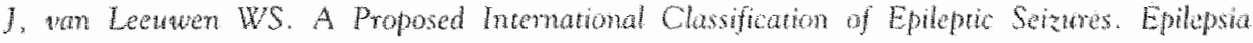
$1964,5: 297-306$.

4. Commission on Classfication and Teminology of the Intemational Lengue Aganst Eptepsy. Proposal for

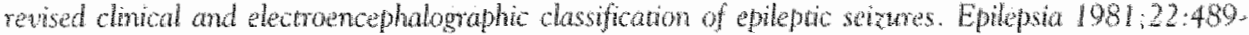
501.

5. Gastant H. Clinical and electroncephalographical dassificamon of eplephic seitures. Eprlepsia $1970,11 \cdot 102-13$

6. Bodensteiner JB, Browwsworth RD, Knapil JR, Kanter MC, Cowan LD, Laviton A. Interwater wariability in the ILAE dassification of seirwes in childhood. Epilepsia 1988;29(2):123-8.

7. wan Donselar CA, Geents AT, Meulste J. Habbma JD, Stal A. Rehability of the diagnosis of a first seizute. Newology 1989;39(2 Pt 1):267-71.

8. Reutens DC, Howall RA, Geber $\mathrm{KE}$, Berkowic SE. Valdation of a questomaire for cinical seizume diagnosis. Eprilepsia 1992;33(6):1065-71.

9. Sander.JW, Shomon SD. Incidence and prevalence studies in epilepsy and the methodological problems: a review. I Newol Newrosurg Psychany 1987,50:829-39

10. Otman R, Lee JH, Haser WA. Reliability of seine classification wing a semismactured intervew. Neurology $1993 ; 43(12): 2526-30$

11. van AstJf, Tatmon JL, Renier WO, Meinardi H, Ahes PPM, Hasman A. Detelopment of diagnostic

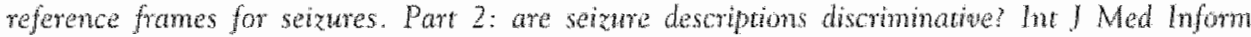
$20036 \cdot 70(2-3): 293-300$

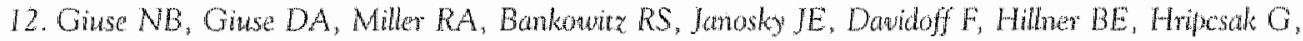
Lincoln M, Middlewon B, Peden Jr. JG. Etaluating consensus among physicions in medical knowledge base construction. Methods In Med 1993:32:137-45.

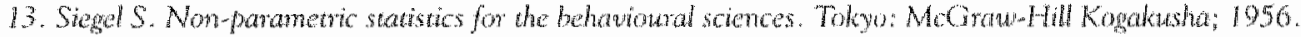

14. Fredman CP, Wyat JC. Evahaton Methods in Medical Informatics. New York Sminger Velag. 1997.

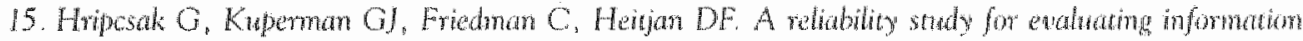
extration from radiology reports. I Am Med lnform Assoc 1999;6:143-50.

16. Bindels R. Hasman A, Wersch JWJ, Pop P, Winkens RAG. The rellability of assessme the appropriateness of requested diagnostic tests. Med Decis Mating 2003; 23(1):31-7.

17. wan der Lei J. Musen MA, wan der Dous E, Man in 'i Veld AJ, wan Bormel JH. Compatison of compateraided and hman review of general practioners managment of hypertension. The Lancet 199) $338: 1504-8$

18. Hastuan A, de Brain LM, Arends. JW. Evaluaton of a method that supponts patholopy report coling. Methods Inf Med 2001:40:293-7.

19. Tuersy A, Kannenan D. Judgment wher uncerainty: hewtistic and biases, Science 1974;185:1124. 31.

20. Feld LS. The approxmate sampling distribution of Kuder-Richardson retiabilin coefficien waeny. Psychomentika 1965;30:357-70. 


\section{Chapter 3}

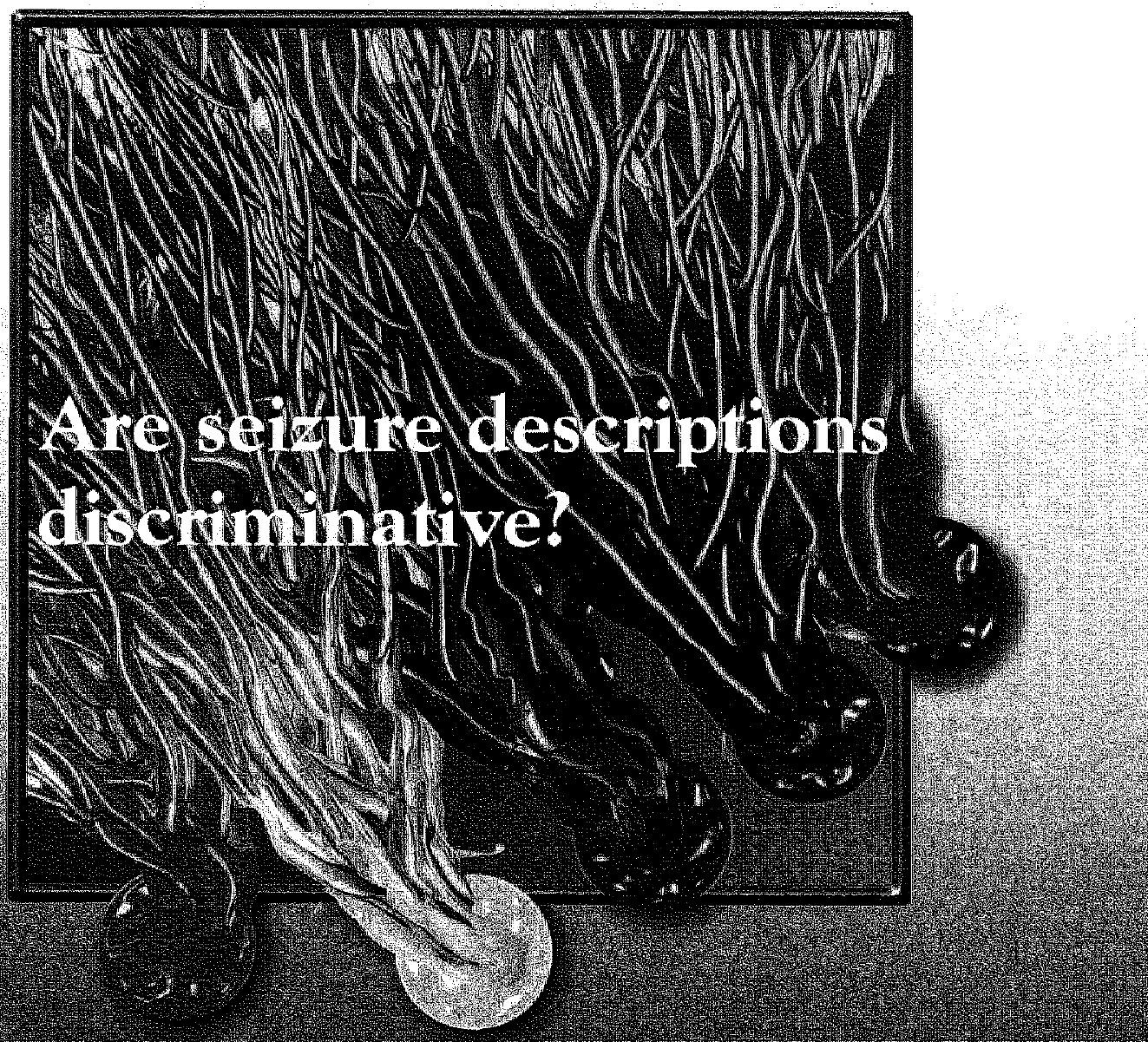

Published as:

van Ast IF Talmon IL, Renier WO, Meinardh H, Alles JPM, Hasman A. Development of diagnostic reference frames for seizures.

Part 2: are seizure deserfprions discriminative?

Intemational Joumal of Medical Infomaties 2003,70(2-3):293-300. 


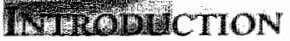

In chapter 2 [1] we concluded that although the neurologists/epileptologists under study do not reach the maximum agreement attainable in the selection of symptoms to describe various seizure types, there exists sufficient agreement on frequently occurring symptoms (present in at least $75 \%$ of the cases) among the participants to construct seizure descriptions. We also determined the number of participants needed to obtain reliable seizure descriptions. In this part we investigate whether the participants selected a discriminative set of symptoms for the various seizure types.

We assume that each seizure type is described by a characteristic ser of symptoms. Because published reports lack univocal descriptions of seizure types, experience of experts has to be taken into account as well. Our hypothesis is that discriminative descriptions of seizure types can be obtained by using the opinions of a group of clinical experts in epilepsy care rather than by eliciting this knowledge from a single expert.

To determine if the seizure descriptions given by each participant are discriminative we compared the symptoms selected by each participant for the seizure types he/she described with templates derived from the descriptions of the various seizure types by his colleagues using a matching score. Secondly we determined to what extent the templates differ from each other by calculating how they are correlated. We also discuss whether our method used is a feasible tool to elicit knowledge from experts.

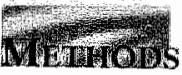

\section{Electronic form}

We collected a list of symptoms associated with seizures from the descriptions of the ICES of 1981 and descriptions of seizure characteristics from other published sources describing seizures [e.g. 2-3]. Two epileptologists (WR, HM) evaluated the list of symptoms with respect to clinical relevance. The symptoms are grouped in 'patient-related characteristics' (27 symptoms), 'clinical manifestations' (130 syhproms) and 'EEG-characteristics' (82 symproms). Each group of symptoms was ordered alphabetically.

The nine most common seizure types were divided in four forms, each consisting of two or three seizure types. These were informally evaluated on their usability prior to data collection. Seizures were grouped according to their position in the hierarchy of the ICES of 1981 (Table 1) on three forms: a) simple partial seizures (SPS) - complex partial seizures (CPS), b) absence seizures (AS) - myoclonic seizures (MS) - tonic-clonic seizures (TCS) and c) typical absence seizures (TAS) - atypical absence seizures (AAS). The fourth form consisted of two seizures that are not at the same level in the hierarchy of the ICES of 1981. As suggested by one of the authors, it is interesting to contrast the symptoms for primary generalized seizures (PGS) and secondary generalized seizures (SGS). These two seizure types are closely related. Since SGS is a partial seizure that develops into a generalized seizure, it is expected that symptoms describing both partial and generalized seizures should

All participants were asked to give comments on the usability of the electronic forms. 
E. Pantial seizures (PS)
A Simple partial seizures (SPS)
B Complex partial seizures (CPS)
C Partial seizures secondary generalized ( 605 ;

$11 . \quad$ Primary Ceneralized seizures (PGS)
A Absence seizures (A5)
1. Typical absence seizures (TAS)
2 Aypical absence seizures (AAS)
B. Myodonic seizures (MS)
C Clonic seizures (CS)
D. Tonic seizures (IS)
E, Tonic-clonic serzures (TCS)
f Atonic seizures (ATS)

III. Unclassified epileptic seizures (US)

Table 1. International Classification of Epileptic Seizures [2]

\section{Analysis}

\section{Discriminative power of seizure descriptions}

As to assess the discriminative power of the seizure descriptions we firstly matched the description for a certain seizure type of one participant with each of the templates for the different seizure types. First, a template $T_{i, l}$ for each seizure type $i, i=1,2, \ldots, 9$ is constructed by counting for each symptom how often it was selected by the participants (excluding the participant whose selection will be analysed). The more participants select the same symptom for a particular seizure type, the more certain we are that the selected sympton is relevant for that particular seizure type. Next, the match, $\mathrm{M}_{k, i}$ between the symptoms selected by participant $k$ for seizure type $\left.i, S_{k, i}=10,1\right\}, 1,1,2$ $\ldots, 239$ and the ones indicated by the template $T_{j,}$ for seizure type $j_{1}$ is computed in the following way:

The score $M_{k, i, j}$ indicates to what extent the symptoms selected by participant $k$ for

$$
M_{k, i, j}=\frac{P_{k, i, j}}{\sum_{i=1}^{239} T_{i, j}} \quad \text { with } P_{k, i, j}=\sum_{i=1}^{239}\left\{\begin{array}{r}
\text { if } T_{j, 1} \neq 0 ; T_{j, 1} \times S_{k, i, 1} \\
\text { else } \frac{-S_{k, i, 1} \times \sum_{i=1}^{239} T_{j .1}}{\sum_{i=1}^{239}\left(1 \mid T_{j, 1}=0\right)}
\end{array}\right\}
$$

seizure type $i$ match the symptoms in the template of seizure type $j$. The 'if' condition in equarion 1 is used to add to the score $P_{k, i, j}$ the corresponding value of the remplate (if this value is not equal to zero) if participant $k$ states that the symprom $S_{k_{i}}$ occurs frequently. The 
"else" condition is used to discount the score each time a participant selects a symptom that has not been mentioned by the other participants as frequendy occurring for seizure type $j$. $M_{k, i, j}$ will be equal to 0 when none or all symptoms are selected by a participant, $M_{k, i, j}$ will be equal to 1 when a participant selects only those symptoms that are selected by at least one other participant and $M_{k, i, j}$ will be equal to -1 when only the symproms are selected that are not selected by any of the other participants $\left(T_{j l}=0\right)$.

Finally, normalized matching scores for seizure type $j, N M_{k, i, j}$, for participant $k$ are determined by dividing $M_{k, i, j}$ by the matching score that participant $k$ obtained for the seizure type he was clescribing $(i)$. This can be computed in the following way:

$$
N M_{k, i, j}=\frac{M_{k, i, j}}{M_{k, i, j}}
$$

The hypothesis is that when consistent and discriminative seizure descriptions have been derived, $N M_{k, i, j}<1$ for every seizure type $j \neq i$.

\section{Correlation coefficients}

Secondly we calculated the correlation coefficients to determine how sets of symptoms of two templates $T_{i, l}$ correlate [4]. We used the following formula:

$$
r_{i, j}=\frac{\sum_{i=1}^{239}\left(T_{i, 1}-\bar{T}_{i}\right) \times\left(T_{j, 1}-\bar{T}_{i}\right)}{\sqrt{\sum_{i=1}^{239}\left(T_{i, 1}-\bar{T}_{i}\right)^{2} \times \sum_{i=1}^{239}\left(T_{j, 1}-\overline{T_{j}}\right)^{2}}}
$$

In this ${ }_{-}$and - are the averages of the scores per symptom for the templates describing seizure typeti and $j$.TThe lower the value of the correlation coefficient the more the templates of the seizure rypes $i$ and $j$ differ in the selected symptoms. If the correlation coefficients are -1 , the templates of the seizure types are each others opposite.

\section{Comments of participants on the electronic form}

Nine of the twenty-four participants gave comments on the usability of the electronic form. They mentioned that the alphabetic ordering of the symptoms made it difficult to consistently indicate related symptoms, e.g., those related to motor signs. Furthermore, in their opinion the terminology used for the description of the "EEG characteristics" was more suited for describing the findings in the EEO in technical terms rather than for describing Anatyse types in clinically meaningful terms. 
For calculating the matching scores we constructed templates for each seizure type. Table 2 gives an overview of the number of symptoms that were selected by $0,1,2, \ldots, 9$ parricipanrs for each seizure type. Secondly, for each seizure type, the participants are ranked in ascending order according to the number of symptoms they selected for that particular seizure type. Figure 1 presents the marching scores for each participant of the symptoms they selected for SPS and the various templates. It can be seen that there is a tendency that the score for SPS increases when the number of selecred symptoms increases. This tendency is observed for all seizure types. By nomalizing the matching scores with respect to those obtained with the SPS template, the discriminative power of the templates can be determined (Table 3 and Figure 2).

By analysing the normalized matching scores (Table 3) we observed two parterns. First, participants can be less successful in idencifying frequently occurring symptoms for the seizure types they chose to describe. This is concluded from the fact that the $N M_{k, i, j} \geq 1$ for one or more seizure types than the one described by these participants (e.g. the match of the CPS symptoms with the templates for AS, TAS and AAS for N15). The second pattern observed was that for some seizure types (almost) all participants have a $N M_{k, i, j} \geq 1$ for another seizure type than the one described by these participants (e.g. for AS).

For SPS, CPS, SGS, MS, TCS and TAS participants (except one or two) were successful

\begin{tabular}{|c|c|c|c|c|c|c|c|c|c|}
\hline lumber of participants & SPS & CPS & PCS & SGS & AS & MS & TCS & TAS & AAS \\
\hline 0 & 90 & 47 & 100 & 52 & 26 & 11 & 65 & 145 & 81 \\
\hline 1 & 61 & 52 & 46 & 58 & 48 & 52 & 49 & 46 & 57 \\
\hline 2 & 29 & 45 & 39 & 55 & 25 & 31 & 34 & 23 & 49 \\
\hline 3 & 25 & 34 & 28 & 40 & 14 & 18 & 32 & 14 & 37 \\
\hline 4 & 17 & 28 & 19 & 23 & 13 & 15 & 30 & 9 & 14 \\
\hline 5 & 8 & 16 & 1 & 1 & 12 & 9 & 17 & 2 & 1 \\
\hline 6 & 5 & 7 & 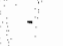 & 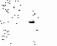 & 1 & 3 & 12 & - & . \\
\hline 7 & 2 & 6 & " & . & - & $=$ & 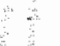 & " & $\therefore$ \\
\hline 8 & 2 & 3 & - & . & $\therefore$ & $=$ & U. & $\because$ & $\therefore$ \\
\hline 9 & 0 & 1 & - & $\therefore$ & $\therefore$ & • & $=$ & " & . \\
\hline
\end{tabular}

Table 2. Number of symptoms selected by $0,1,2, \ldots, 9$ participants

in identifying discriminative frequently occurring symptoms. For example, N15 and N19 were less successful in identifying discriminative frequently occurring symptoms for SPS (Figure 1 and Figure 2).

For the aggregated generalized seizure types PGS and AS participants selected symptoms that better matched with TCS and TAS respectively. It is observed that the symptoms selected by four of the five participants for PGS have an equal or better march with the TCS templare (marching scores of 1.00-1.24). The symptoms selected by the fifth participant better matched the templates of both MS and (T)AS. A similar result was observed for AS: for all participants, the symptoms selected for AS better matched the TAS template (matching scores of 1.02-1.34). Note that different groups of experts selected symptoms for AS and TAS. This was also the case for PGS and TCS. 


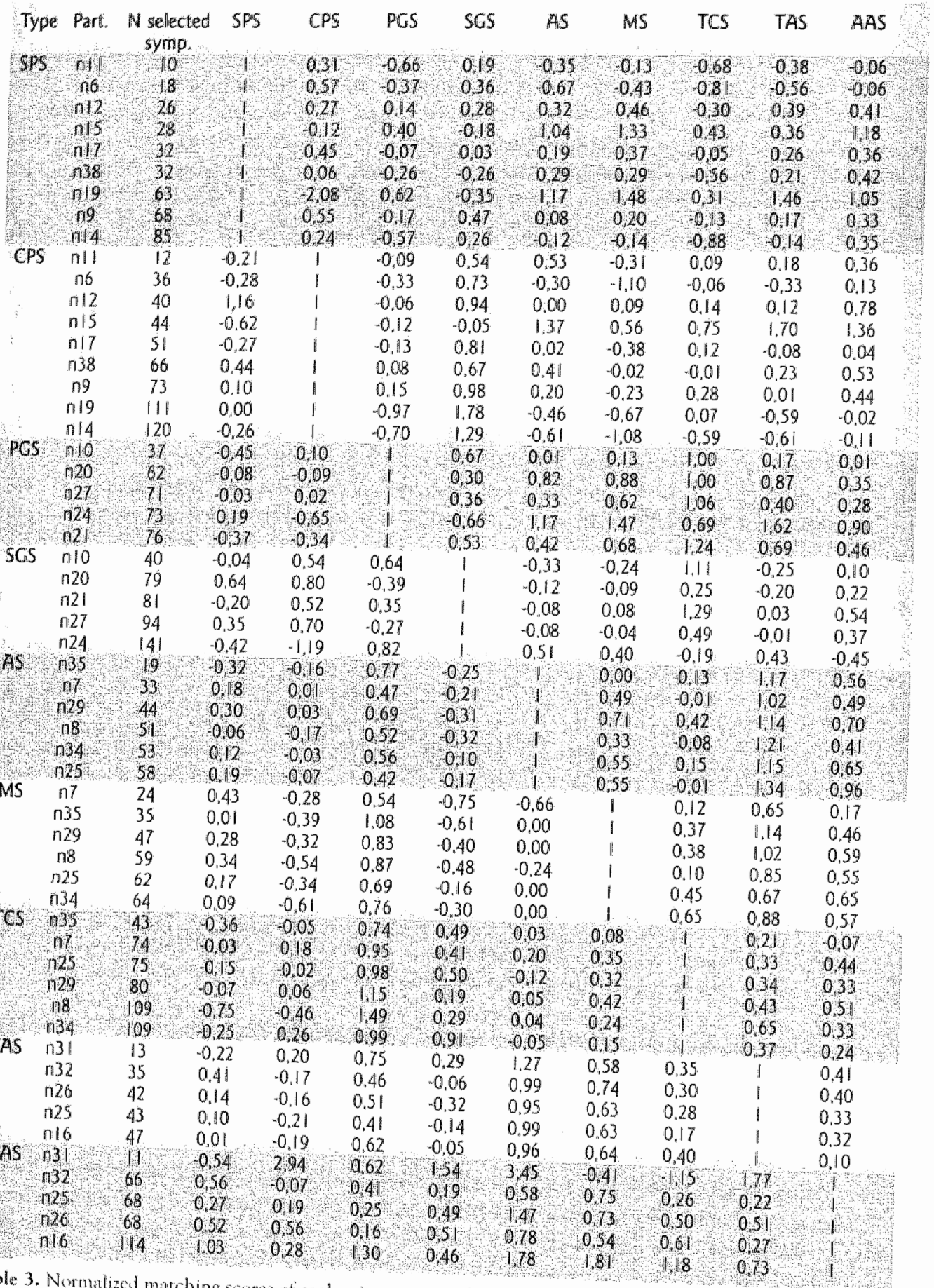

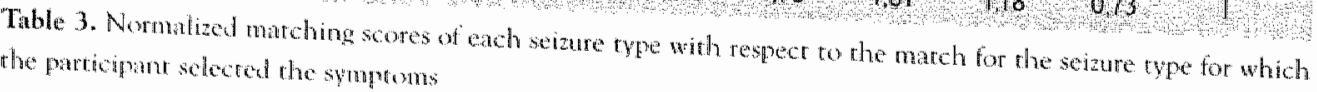


Three of the five experts had difficulties in identifying frequently occurring symptoms for AAS.

The correlation coefficients between the templates of the seizure types are presented in a correlation matrix (Table 4). The correlation coefficients between the templates of PGS. TCS and AS-TAS are relatively high (0.67 and 0.77 respectively) compared to the correlarions between the orher seizure types.

Participants indicated that it was difficult to select frequently occurring 'EEG-characteristics'

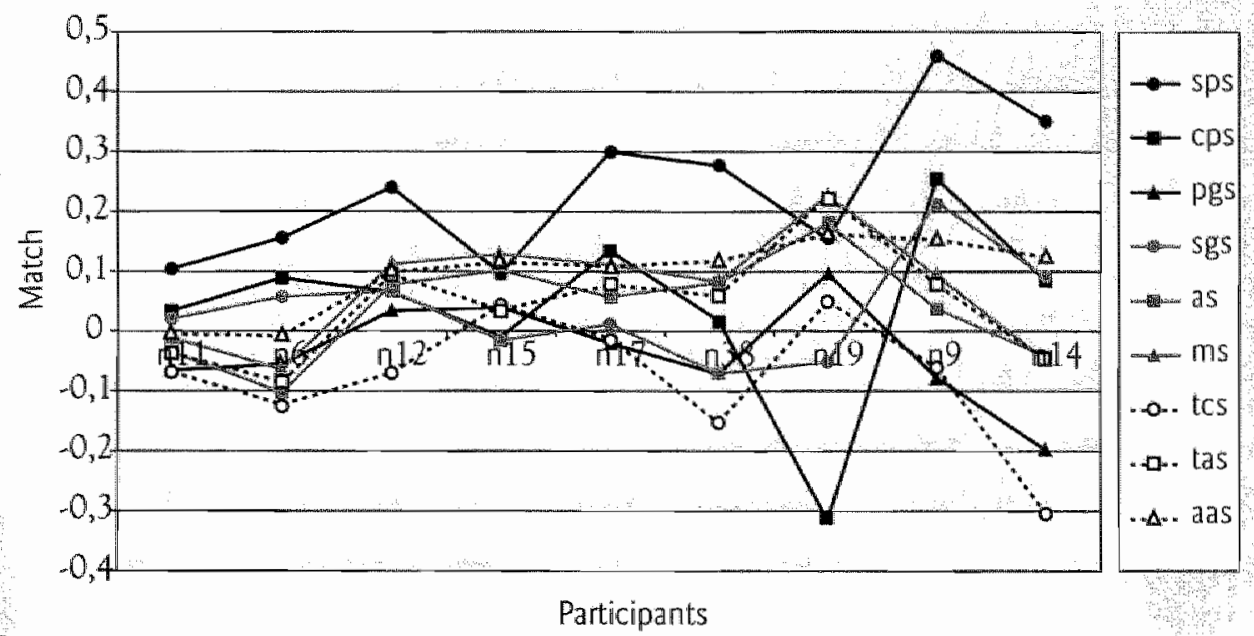

Figure 1. Match (y-axis) between symptoms selected by the parricipants for SPS and the symptoms indicated on the cemplates. Participants are ranked according to the number of selected symptoms ( $x$-axis)

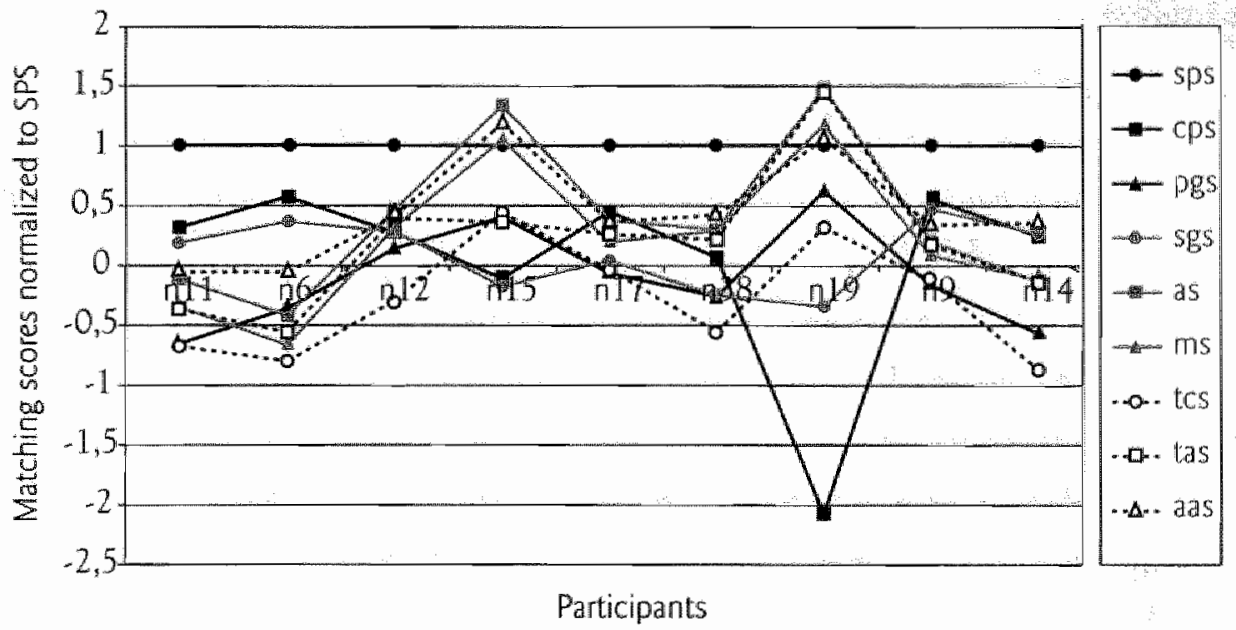

Figure 2. Matching scores for SPS normalized wirh respect ro the match with the SPS template 
for the various seizure types. Therefore we calculated the matching scores and the correlation coefficients excluding the 'EEG-characteristics'. Removing the "EEGcharacteristics' did not radically change the matching scores. However, five correlation coefficients between the templates of the seizure types significantly decreased when the "EEG-characteristics" were excluded (Table 4). The correlation coefficients between SGSPGS and SGS.TCS significantly increased (from 0.27 to 0.48 and 0.37 to 0.55 respectively). In the correlations between the other seizure types no significant increases and decreases were observed.

\begin{tabular}{|c|c|c|c|c|c|c|c|c|c|c|}
\hline Ind.EEC & Excl.EEG & SPS & CPS & PGS & SES & AS & M5 & TCS & TAS & AAS \\
\hline SPS & & $x$ & 0,229 & 0.080 & 0,119 & 0,100 & 0.263 & 0,128 & 0.090 & 0,282 \\
\hline CPS & & 0,307 & $x$ & 0,027 & 0,484 & 0,016 & 0.176 & 0,140 & -0.043 & 0,243 \\
\hline PGS & & 0,082 & 0,009 & $x$ & 0.480 & 0.238 & 0,397 & 0,668 & 0,301 & 0.18 \\
\hline SGS & & 0,159 & 0.438 & 0.274 & $x$ & 0.115 & 0,060 & 0.553 & 0.045 & 0.147 \\
\hline AS & & 0,085 & 0,003 & 0,364 & 0116 & $x$ & 0,322 & 0,084 & 0.718 & 0.437 \\
\hline 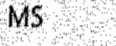 & & 0,170 & -0.150 & 0500 & -0.074 & 0.489 & $x$ & 0.231 & 0.404 & 0.244 \\
\hline$g$ & & 0.144 & 0,089 & 0.671 & 0,365 & 0.228 & 0,383 & $x$ & 0.103 & 0.151 \\
\hline$A$ & & 0.110 & 0,028 & 0.430 & 0.072 & 0.771 & 0,526 & 0.243 & $x$ & 0.361 \\
\hline & & 0,238 & 0,198 & 0.191 & 0,193 & 0.431 & 0.314 & 0,166 & 0390 & \\
\hline
\end{tabular}

In bald and thalies significantichanges when EEG-characteristics are excluded from the calculation of the correlation coefficients

Table 4. Correlation coefficients between seizures including EEG-characteristics (below the diagonal)and axcluding EEG-characteristics (above the diagonal)

\section{Drscussion}

\section{Selection of symptoms}

The analyses show that participants select a discriminative set of frequently occurring symptoms for the various seizure types, although the selection of symptoms for differentiating the aggregated seizure types PGS and AS appears to be problematic.

Theoretically, the selected symptoms for PGS must be a subset of the symptoms selected for TCS, MS and (T)AS. Symptoms occurring in more than $75 \%$ of the cases in, for example, TCS should only be selected for PGS if they also occur sufficiently often in AS and MS; otherwise they cannot occur in $75 \%$ of the PGS cases. Moreover, symptoms selected for PGS should still have a better match with the PGS template than with the TCS template. However a more or less equal match of the selected symptoms with the PGS and TCS template was observed. We assume that the neurologists/epileptologists have taken one of the subtypes of PGS (very likely TCS) as a prototypical case for PGS; hence the similar templates. When the neurologists/epileptologists selected symptoms for AS we assume that they have taken TAS as a prototypical case for AS. The correlation coefficients between the templates of PGS-TCS and AS-TAS support these assumptions. We concluded 
that participants have difficulties in selecting specific symptons for aggregated seiatre npes. Although PGS and AS occur as entities in the international classification of epteptics seizures (ICES), they are not used in clinical practice. The specific seizure types, like TCS and TAS, are used to classify the seizures of a patticular patient.

The contrast between symptoms for primary generalized seizures (PGS) and secondary generalized seizures (SGS) was also studied. Results show that almost all participants selecting symptoms for SGS have higher matches with the templates for CPS and TCS. This was expected, although a SGS may start as a SPS, they often start as a complex partial seizure and all become finally a generalized seizure (mostly TCS). For PGS, participants selected symptoms that had a low match with the templates of the partial seizures. Obviously participants were able to distinguish PGS from SGS and were able to describe $P G S$ and $S G S$ in a meaningful way.

\section{Matching scores}

There are several ways to calculate the matching scores between symptoms sellected by a participant and the seizure templates. In our study we chose to 'punish' a participant when he/she selected symptoms that none of the other participants selected (see 'else' condition in equation 1). When a participant selects a symptom that is also selected by at least one of the other participants, the value of the template for that symptom is added to the score. We found a tendency that the matching scores increase when the number of selected symptoms increases. Apparently the participants who selected a lot of symptoms selected the same symptoms as at least one other participant. Furthermore, we scaled the matching score between +1 and -1 . Our matching works only when there are quire a few symproms that are not selected by any of the participants. This is the case in our study as is seen from Table 2. When there are only a few symptoms not selected by any of the participants, the punishment score becomes large. Selecting any of such symptoms will have a severe impact on the resulting score.

As an alternative, we also computed the correlations between a neurologist's profile and the various templates. This analysis gives essentially the same results.

\section{Lessons learned}

The study reported here was carried out to determine the feastbility of eliciting desoriptions of seizure types from experts. The lessons we learned from this feasibility study will be taken into account in the final study.

In chapter 2 [1] we already mentioned that differences in knowledge and experience of the patticipants could explain part of the variability in the selection of the symptoms. Some symptoms were multi-interpretable which could cause variability in the selection of these symproms. But due to the small number of neurologists/epileptologists who participated in the study, this hypothesis could not be tested. Definitions of symptoms will be offered to the participants in the final study.

The second source of variability in the selection of symptoms may have been caused by the characteristics of the study. Participants were asked to identify symptoms they considered present in more than $75 \%$ of the cases. The threshold of $75 \%$ is probably difficult: to assess, resulting in relatively many symptoms that were only selected by one or two participants. Also rarely occurring symptoms that are highly specific for a particular seizure type are not obtained in this way. These symptoms are meaningful for the construction of 
DRFs because they have a high discriminatory power. In the final study therefore we will ask neurologists/epileptologists to estimate, on a 5-point scale, the frequency of occurrence of the symptoms in each seizure type.

It is likely that inconsistencies in the selection of symptoms cam be further decreased when neurologists/epileptologists are asked to re-assess their answers for the symptoms on which the judgements of the participants varied most. Such a Delphi-like approach will therefore be used in the final study.

Our anallysis revealed that neurologists/epileptologists have difficulties in consistently identifying the relevant symptoms for aggregated seizure types. Although these aggregated seizure types are useful to categorize seizures, they have limited clinical value. Since we are interested in descriptions of seizures that are relevant for clinical practice we will restrict the knowledge acquisition in the final study to the following seizure types: SPS, CPS, MS, TCS, TAS and AAS (see Table 1).

The electronic form implements a structured data collection method with fixed items. The advantage of this method is that greater uniformity in the reporting of relevant symptoms can be achieved, under the condition that the symptom names carry the same meaning for all participants. An electronic form can also be administered relatively easy to larger numbers of participants [5]. The comments of the participants on the electronic form and on the items on the list of symptoms provided suggestions for revision and improvement of the form for the final study. We reported that the participants found it difficult to select frequently occurring 'EEG-characteristics' for the various seizure types. This can be caused by the fact that surface EEG's do not always correlate with discharges from deeply located generators. Based on the comments of the participants we expected that the correlation coefficients would decrease between all templates when the "EEG. characteristics' were excluded from the calculations. However, the correlation coefficients between the templates of SGS and PGS and of SGS and TCS significantly increased. The 'EEGcharacteristics' appear to contain useful information for discriminating these seizure types, even though participants reported difficulties in selecting 'EEG-characteristics'. Therefore the characteristics describing the EEG patterns in detail (such as ictal frequency in $\mathrm{Hz}$, (inter-)ictal discharges and (inter-) ictal EEG activity) cannot be left out because they add to the discriminatory power, but should be replaced by more general terms (such as characterization and localization of epileptic discharges). lnstead of an alphabetical ordering of symptoms, a clustering of symptoms will facilitate access to them and hence ease filling in the electronic form.

The ILAE presented a "Proposed diagnostic scheme for people with epileptic seizures and with epilepsy" during the $24^{\text {th }}$ International Epilepsy Congress [6-7]. This scheme consists of five axes. The first axis consists of a list of ictal phenomena presented as a "Glossary of descriptive ictal terminology". The purpose of this glossary is to provide standard terminology. Practitioners about a seizure [6]. The list of symptoms we developed can also be considered as a terminology representing the domain of concepts for describing epileptic seizures. In future studies we shall harmonize our list with the proposed "Glossary of descriptive ictal terminology".

\section{MoNG IMISION}

discriminative show that the participants in our study selected symptoms that result in discriminative descriptions of the seizure types. This indicates that knowledge elicitation by using the opinions of a group of clinical experts is possible. The descriptions of aggregated 
seizure types, such as Primary Generalized Seizures, are less discriminatory than the descriptions for more specific seizure types. An electronic form is a feasible tool for collection of this rype of information.

We expect that for the revised design of our study we need less than the 40 neurologists, predicted from the results of our current study, for knowledge elicitation. 
Chapter 3 


\section{6) HER}

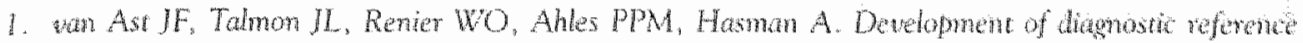

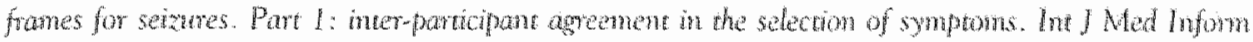
$2003: 70(2-3): 285-202$

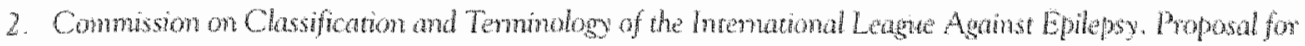

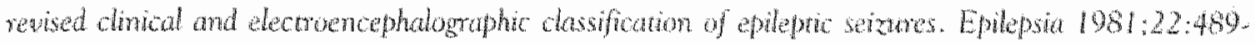
501.

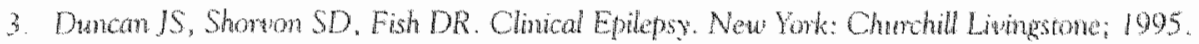

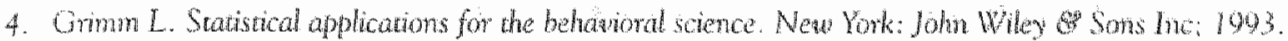

5. Kerlinger F, Lee H. Foundatons of behaworal resanch. Otanda: Harcumt College Wublishes; 2000.

6. ILAE Task Fore on Clasification and Teminology. A proposed diagnoste schena for people with

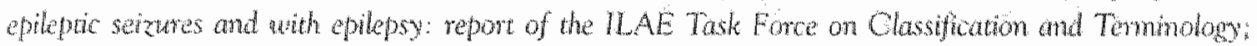
2001 watur.epilepry. otg.

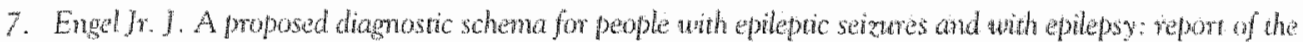
ILAE Task Force on Classification and Temmology. Epilepsia 2001:42:796-803. 



\section{Chapter 4}

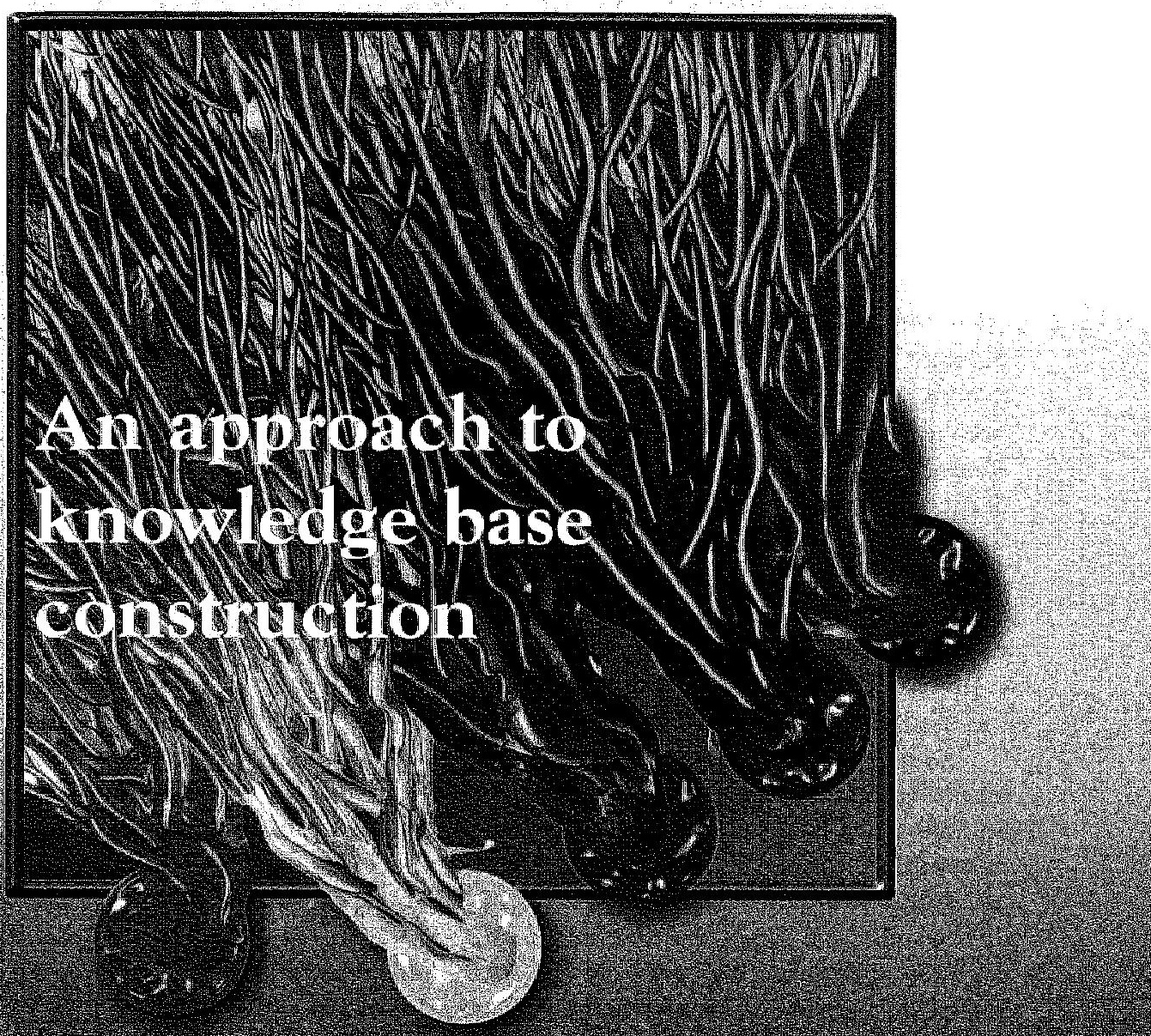

Poblished as:

van Ast IE, Talmon L, Renier WO L Lasman A.

An approach to knowledge base construction baged on expert opinions: Methods of Information in Medicine 2004, 43.427 32. 


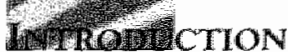

There are various kinds of decision support systems. For a decision support system that works with probabilities and Bayes' rule, the use of knowledge from databases and/or the literature is preferred. In practice the situation often arises that knowledge about a number of (semi-) quantitarive items is needed in a certain application but not available in databases and/or the literature. Knowledge acquired from a single expert may not be reliable [1] and therefore it is better to obtain the knowledge from a number of experts.

Giuse et al. evaluated the inter-author agreement in knowledge base construction among seven internists by comparing the observed agreement among the participants with the agreement expected by chance. They concluded that agreement among the majority of the participants was significantly higher than predicted by chance [2]. In a pilot study we used the same method as described by Giuse et al. and concluded that knowledge elicitation by using opinions of a group of expert is possible [3].

Criteria are needed to determine how many experts are needed so that the average of their estimates for the value of each item is reliable. In this case the inter-rater intraclass correlation coefficient (ICC) used in the behavioral sciences can provide help. The ICC operationalizes the reliability concept. It is a value between one and zero that indicares the fraction of the variance in the set of item values (each item is scored by the participating experts) that is due to real differences between items and not to random or systematic estimation errors of the experts. An ICC of one indicates that no random or systematic errors have been made. Usually an ICC of 0.7 or more is an indication of a good reliability of the ser of data. If one is interested in the reliability of each item separately ICC values of 0.9 or more are advised $[4,5]$.

The extent of random and systematic errors varies with the problem domain. In a socalled measurement study one can calculate the ICC of the data estimated by a number of experts. If this ICC is not large enough (the contribution of random and systematic errors is too large) one can determine how many additional experts are needed to obtain a pre-
specified ICC.

In practical situations, if no measurement study is available, one first collects data from a number of experts and assesses the reliability of the data. If the reliability is not good enough the number of additional experts needed to obtain the pre-specified ICC is determined, using the available information. These additional experts may come from different institutions and may be even from different countries. Then two questions arise: do the additional experts deviate from the opinions of the existing group of experts (in other words: are the groups samples from different populations) and are there deviating opinions within the total group of experts. Identification and removal of these experts may increase
the reliability of the data.

In this paper we discuss how we derermine the reliability of the estimates of the frequency of occurrence of manifestations in various seizure types provided by neurologists/ epileptologists [6] and present a general approach to identify participants with largely
deviating opinions.

In the following sections the term manifestation will be used to indicate both signs and
ptoms. 
In this section we describe the approach to identify participants with largely deviating opinions and how we applied this approach to the data provided by neurologists/epileptologists. The approach is applied after all opinions of all experts have been collected.

First is checked with the help of method A (see following section) whether the opinions of the additional experts as a group are inconsistent with those of the initial group. Individual inconsistencies, which may be due to 'outliers' in the initial group, but also due to deviating opinions of individual additional experts are identified and further studied with method $B$. Merhod $B$ is applied to the total group to identify experts with deviating opinions. If such an expert is identified, the contribution of this expert is not taken into account. Not including the results of this expert should increase the ICC even though there is one expert less. If the total group of experts does not show any deviating experts but a lower relialsility than expected on the basis of the calculations, this may be due to the fact that the additional experts came from another 'population'. This fact however has then already been determined by method A.

\section{Approach}

The approach consists of applying two methods ( $\mathrm{A}$ and $\mathrm{B}$ ), as explained above. The dataset exists of opinions of a group of experts who estimated the frequency of occurrence of manifestations for various seizure types. The inter-rater intraclass correlation coefficient (ICC) is used to determine the reliability of the data. The Statistical Package for the Social Sciences (SPSS version 10.0) was used to calculate the ICC. More information about the $1 \mathrm{CC}$ can be found in appendix I.I.

\section{Method A of the approach}

To assess whether a new participant provides information that is consistent with the information already provided by a group of participants ( $n$ participants) we developed the following procedure:

- Use the set of data of $n$ participants to calculate the ICCs for the $n$ different sets of $n-1$ out of $n$ participants;

- Estimate the standard deviation (SD) of the calculated ICCs;

- Apply the Spearman-Brown prophecy formula (see appendix II) to the ICC based on $n$ participants to predict the ICC for $n+1$ participants [5];

- Apply \pm 2 SD around the predicted ICC for $n+1$ participants to determine the confidence interval for the predicted ICC for $n+1$ participants;

- If the actual $1 C \mathrm{C}$ for $n+1$ participants falls within the confidence interval of the predicted ICC for $n+1$ participants the new participant provides information that is consistent with the information given by the group of participants.

\section{Notes:}

1. SPSS also provides a confidence interval for the ICCs. This value cannot be used in our approach because we do not have independent samples.

2. We assume that the SD does not differ between $n$ and $n+1$ participants.

\section{Method B of the approach}

To determine for each participant whether he/she provides information that is consistent with the information of all other participants the following procedure is developed: 
- Use the set of data of all participants to calculate the ICCs for the m different sets of $m-1$ participants;

- Estimate the standard deviation (SD) of the calculated ICCs after excluding the set that did not contain participant $k$;

- Apply the Spearman-Brown prophecy formula (see appendix. II) to the ICC based on $m-1$ participants (excluding participant $k$ ) to predict the $10 C$ for $m$ participants [5].

- Apply \pm 2 SD around the predicted ICC for m participants to determine the confidence interval for the predicted ICC for $m$ participants;

- If the actual ICC for marticipants falls within the confidence interval of the predicted ICC for m participants, participant $k$ prowides consistent information.

\section{Material}

The approach described in the sections 2.1.1 and 2.1.2 is used to identify neurologists/ epileptologists with diverging opinions about the frequency of occurrence of manifestations in epileptic seizures.

Neurologists/epileptologists were asked for one or more seizure types to estimate how often manifestations occur using a 5 -point scale ranging from 1 = nearly not occurring $(<20 \%)$ to $5=$ nearly always occurring $(>80 \%)$. We addressed the most frequently occurring seizure types relevant in clinical practice: simple partial seizures (SPS), complex partial seizures (CPS), myoclonic seizures (MS), ronic-clonic seizures (TCS), typical absence seizures (TAS) and atypical absence seizures (AAS). The same list of manifestations consisting of 156 manifestations - was used for each seizure type. The list of manifestations was included in an electronic form $[3,7]$.

To obtain reltable seizure descriptions we asked a group of Dutch neurologists/ epileptologists no fill out the electronic form between December 2001 and June 2002. Seventeen were willing to participate and finally ten Dutch neurologists/epileptologists filled out the electronic form for one or more seizure types. In a Delphi-like procedure the Dutch participants were asked to review their responses focusing on manifestations with divergent responses (September 2002).

We used the inter-rater ICC to assess how homogeneous the Dutch participants were in estimaring the frequency of occurrence for each manifestation in each seizure type. If the ICC $\geq 0.915 \mid$ we conclude that the averaged frequency estimate of the participants for each manifestation within a given seizure type is relable. The inter-rater ICG increases when the number of observers increases assuming they share a common view. So given the $1 \mathrm{CC}$ obtained with a certain number of observers, one can use the Spearman-Brown prophecy formula to calculate the $1 \mathrm{CC}$ for any other number of observers or the number of observers needed to get a certain $1 \mathrm{CC}[5]$.

To reach the predicted number of participants, needed for an ICC of 0.9 , we extended. when necessary - our panel of experts with neurologists/epileptologists who attended the $5^{\text {th }}$ European Epilepsy Congress in October 2002. Neurologists/epileptologists were asked to fill out. the electronic form for at least one seizure type. Because of the situation the Delphi-like procedure among the congress participants was different from the one used for the Dutch neurologists/ epileptologists. After completing the electronic form, the congress participants were asked to directly review their responses for the ten manifestations with the largest normalized difference from the mean freouency estimate based on the responses of the Dutch participants. 


\begin{tabular}{|c|c|c|c|c|c|c|}
\hline $\begin{array}{l}\text { Selzune } \\
\text { type }\end{array}$ & $\begin{array}{l}\text { Dut } \\
\text { Dutch } \\
\text { patt }\end{array}$ & $\begin{array}{l}\text { ICC for } \\
\text { Dutch } \\
\text { participants }\end{array}$ & $\begin{array}{l}\text { Apart needed for } \\
\text { lCC } \geq 0,90 \text { based on } \\
\text { Dutch panticipants }\end{array}$ & $\begin{array}{l}\text { congress: } \\
\text { part }\end{array}$ & $\begin{array}{l}\text { Total part } \\
\text { (Dutchtcongress) }\end{array}$ & $\begin{array}{l}\text { Find } \\
\text { CCC }\end{array}$ \\
\hline SPS & 9 & 0.849 & 14 & 4 & 13 & 00875 \\
\hline CPS & 10 & 0851 & 16 & $4^{*}$ & 14 & 0,880 \\
\hline $\mathrm{MS}$ & 9 & 0.876 & 月 & 3 & 12 & 09515 \\
\hline TCS & 8 & 0.897 & 8 & 2 & 10 & 0915 \\
\hline TAS & 7 & 0926 & 5 & 0 & 1 & 0926 \\
\hline AAs & 1 & 0778 & 18 & 2 & 9 & 0,782 \\
\hline
\end{tabular}

Table 1. Number of Dutch and congress participants for each seizure type with the corresponding ICC-values

\section{Experiments}

The following experiments were performed:

\section{Experiment 1}

Method $\mathrm{A}$ of the approach was used to assess whether the congress participants provided responses that were consistent with those of the Dutch participants.

\section{Experiment 2}

Method B of the approach was used to assess whether in the total group there are participants with opinions that are not consistent with the responses of all other participants.

\section{Experiment 3}

To estimate the power of the approach (the probability that the null hypothesis is correctly rejected) we added the responses of congress participants estimating the frequencies of other seizure types to the responses provided by the Dutch participants for a particular seizure type. In the ideal situation the resulting ICCs should all fall outside the confidence interval of that particular seizure type. The power is estimated by dividing the number of ICCs that indeed fall outside the confidence interval by the total number of ICCs calculated using the responses of the wrong seizure types. If the power is equal or larger to 0.8 one can assume that the approach correctly identifies consistent responses.

\section{Experiment 1}

For complex partial seizures, myoclonic seizures, tonic-clonic seizures and atypical absence seizures all ICCs for the Dutch participants plus one congress participant fell within the 


\section{Chapter 4}

range of $\pm 2 S D$ around the predicted $1 C C$ for $n+1$ participants (Figure 1). Two congress participants provided slightly different responses compared to the group of Dutch participants for simple partial seizures. For these two participants the ICC was 0.8479 and 0.8485 respectiwely while the confidence interval for the predicted $n+1$ participants ranged from 0.8487 to 0.8753 . For typical absence seizures no congress participant estimated the frequency of occurrence of manifestations.

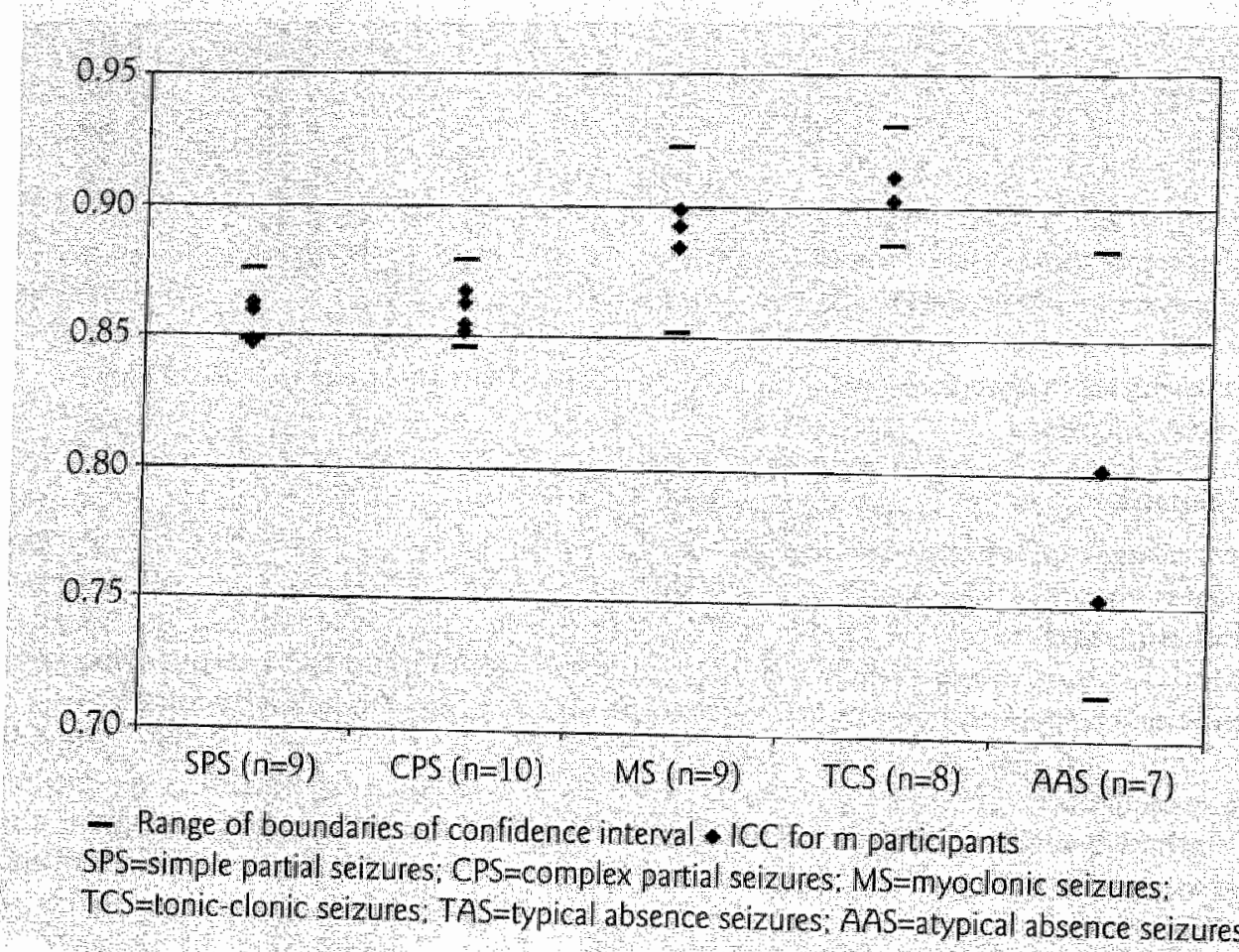

Figure 1. Predicted confidence intervals (CI) for $n+1$ participants and the observed ICC for $n+1$ partipants for
each seigure type

\section{Experiment 2}

We applied method B to the total group of participants for each seizure type and investigated whether each participant provided responses that were consistent with the responses of the whole group. We observed that one Dutch participant $(k 14)$ differed in his/her responses for myoclonic seizures, typical absence seizures and atypical absence seizures. By excluding this participant from the data the final ICC increased (Table 2). The result for myoclonic seizures are presented as an example in Figure 2. For the other seizure types the frequency estimates provided by the participants were consistent with the data provided by the whole group of participants. Also the two slightly deviating responses as identified in experiment
1 were not present when applying method $B$. 


\section{Experiment 3}

To calculate the power of method $A$ we excluded the diverging responses of participant $k 14$ in myoclonic seizures, typical absence seizures and atypical absence seizures to obtain a homogeneous group.

Seizure type

Myoclonic seizures

Typical absence seizures

Atypical absence seizures
ICC including participant $\mathrm{k} / 4$

0.9150

09260

0.7820
ICC excluding participant $\mathrm{k} / 4$

0.9298

09388

0.8172

Table 2. Increase of ICC when participant $k 14$ is excluded

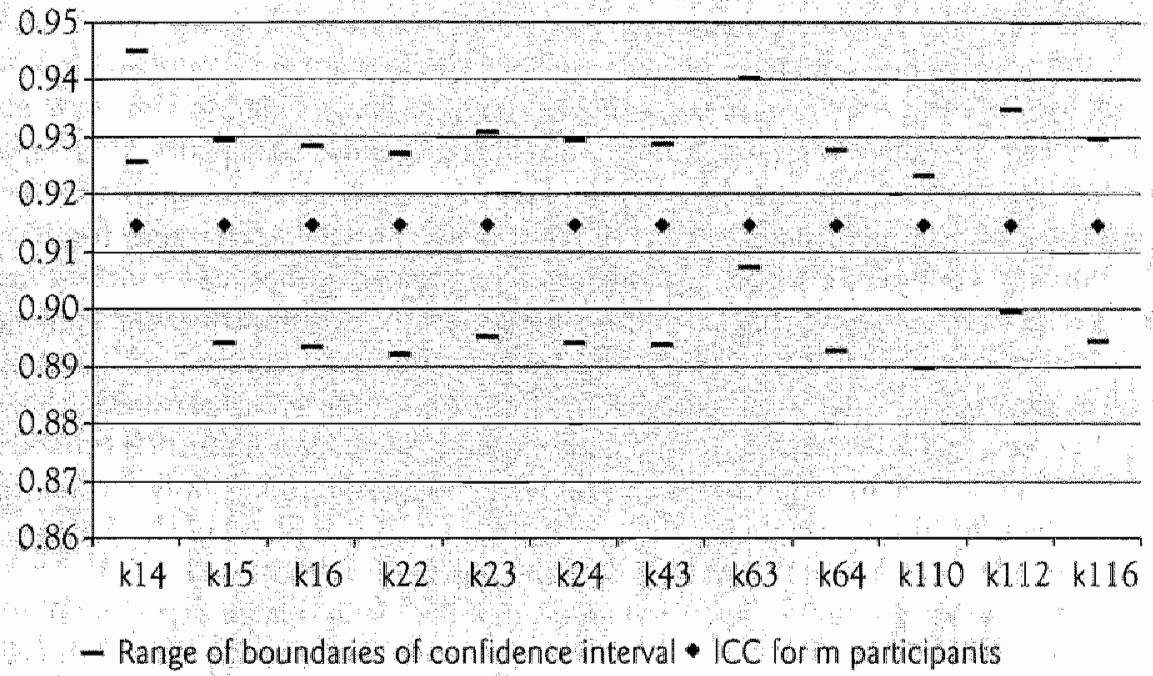

Figure 2. Predicted confidence intervals (Cl) for m participants and the otserved $\mathrm{CO}$ for $m$ participunts for myoclonic seizures $(m=12)$

Seizure type

Simple partial seizures

Complex partial seizures

Myoclonic seizures

Tonic-clonic seizures

Typical absence seizures

Atypical absence seizures
Power of the approach

$$
\begin{gathered}
1 \\
0.91 \\
0.75 \\
1 \\
0.93 \\
0.92
\end{gathered}
$$

Table 3. Power of method A (for explanation see the text) 
The power of the approach varied between 0.75 for myoclonic seizures and 1 for simple partial seizures and tonic-clonic seizures. Table 3 gives an overview of the power for each of the seizure types.

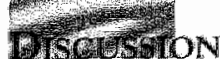

The approach to identify participants with diverging responses turned out to be feasible, although method $B$ is rather time consuming. The exclusion of an identified participant with diverging responses improved the reliability of the data.

In this paper we only present an approach to assess if reliable data are obtained in a knowledge acquisition procedure. Reliable data do not have to be valid. The validation of the obtained knowledge is discussed in another paper. However, a good reliability is a prerequisite for a good validity and therefore should be scudied first.

\section{Reflection on the methods}

The assessment of the reliability was carried out - as stated earlier - after the data acquisition phase. If the assessment would have been carried out during the data acquisition phase, e.g. to decide whether the responses of a participant have to be included in the study or not, there is a risk of locking in on the opinions of the first group of participants. Hence, we did not use this approach for sequential inclusion of participants. In the study reported here we gathered the data first and finally assessed if participants provided consistent responses.

The initial group formed our starting point and was not directly checked for inconsistencies. We first wanted to know whether the additional participants came from the same population as the initial group. The quality of the initial group is checked with method B and indeed it could be shown that one of the participants from the initial group was an outlier. The slightly diverging responses as determined with method $A$ were not identified by method $B$ on the larger group, again supporting our strategy of using method A only for identifying possible outliers but not for removing their contributions in this phase of the process.

If a participant according to method B of the approach is indicated as an outlier the ICC-value for the total number of participants will either be too large or too small for the confidence interval. When this ICC is too large the contribution of the participant should not be deleted, because his/her answers are strongly consistent with the other opinions. If the ICC is too small the contribution of the participant should be deleted. We identified one outlier coming from the initial group.

Method B can also be used instead of method A to determine whether the additional experts differ from the initial group. We repeated experiment 1 with method $B$. The same results were obtained: the participants with slightly different opinions that were identified with method $A$ were also detected by method $B$. This would imply that method $A$ is not necessary. However, method $B$ is more complex and more time-consuming and therefore we introduced method $\mathrm{A}$.

The power of method $A$ of the approach is good $(\geq 0.75)$, which indicates that the method correcty states that a new participant is consistent with the participants in the initial group. The power of method $B$ can also be estimated by again adding the responses of a participant estimating the frequencies of a seizure type to the responses provided by the orher participants for another seizure type. Because of the time needed to assess the power of method $B$ for each seirure type we added a random sample of five participants each 
prowiding data from another seizure type to two selected seizure types (simple partial seizunes and myoclonic seizures). All ten cases correcty fell outside the confidence intervals. Because this random sample resulted in a power of one for both seizure types and the tact that method $B$ provided the same results as method $A$, we assume that the power of method $B$ for each seizure type is equally good.

\section{Application of the methods to the seizure type domain}

To obtain reliable seizure descriptions we needed an $1 \mathrm{CC}$ equal or larger than 0.9 . For simple partial seizures (rounded ICC), complex partial seizures (rounded ICC), myoclonic seizures, tonic-clonic seizures and typical absence seizures this $1 \mathrm{CC}$ is obtained. For atypical absence seizures we could not include enough participants to obtain an $1 \mathrm{CC} \geq 0.9$; only two congress participants filled out the electronic form, while eleven more participants were needed.

Only two congress participants marginally differed in their responses from the Dutch participants for simple partial seizures. It could be concluded that the congress participants as a group did not differ in their responses from the Dutch participants. Apparently neuro. logists/epileptologists from different countries have similar ideas about the characteristics of the seizure types investigated.

We identified one participant (k14) who deviated in his/her responses from the other participants for myoclonic seizures, typical absence seizures and atypical absence seizuras. After this participant was excluded from the analyses the ICC increased in all three cases.

Based on the responses of the Dutch participants it was possible to estimate the number of participants needed to obtain an $\mathrm{ICC} \geq 0.9$ using the Spearman-Brown prophecy formula. The estimated number will only be correct if the neurologists/epileptologists form a single population. The fact that the estimated ICC was close to the anticipated value is also an indication of the fact that there are no big differences between the Dutcth and congress participants.

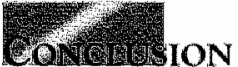

We presented an approach to create a knowledge base based on opinions of experts. We concluded that the knowledge base was reliable. Moreover we could show that the congress participants and the Dutch participants appear to be samples from the same population. We also identified one participant with diverging opinions about the frequency of occurrence of manifestations in some seizure types. Eliminating the contribution of this participant led to a higher reliability. 
Chapter 4 


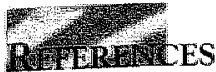

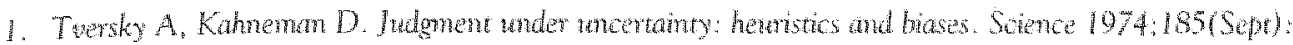
1124.1131

2. Guse NB. Giuse DA, Mhler RA, Bankowiz RA, Janoky IE, Dowdolf F. Hilner BE,

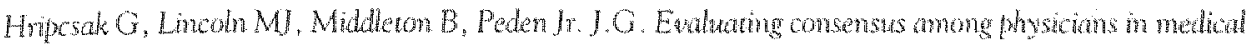
knowledge base consmaction. Methods Inf Med 1993,202):137.45

3. wan Ast JF, Talmon IL. Rener WO. Ahes PPM. Haman A. Dewelopmenc of dagnosic nefuence fromes for seizates. Part I: imter-participant agrement in the selection of symproms. Int of Med Inf $2003 ; 70(2-3): 285-92$.

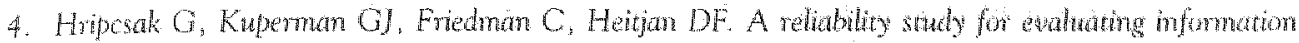
exraction from radology reports. I Am Med Inform Assoc 1990,6(2):143-50.

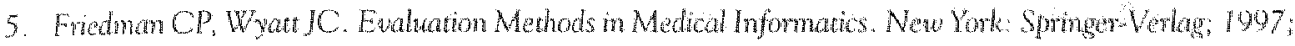
Chapter 5 .

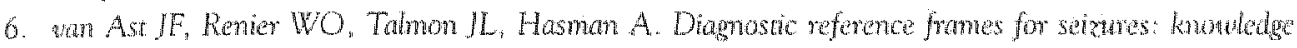
elicitution and restuls. 2004:Submitred.

7. wan Ast JF, Talmon IL. Renier WO, Meinardi H. Ahles PPM, Hasman A. Dewelopmen of diagnostic

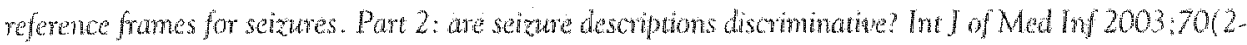
3):293-300. 


\section{Chapter 5}

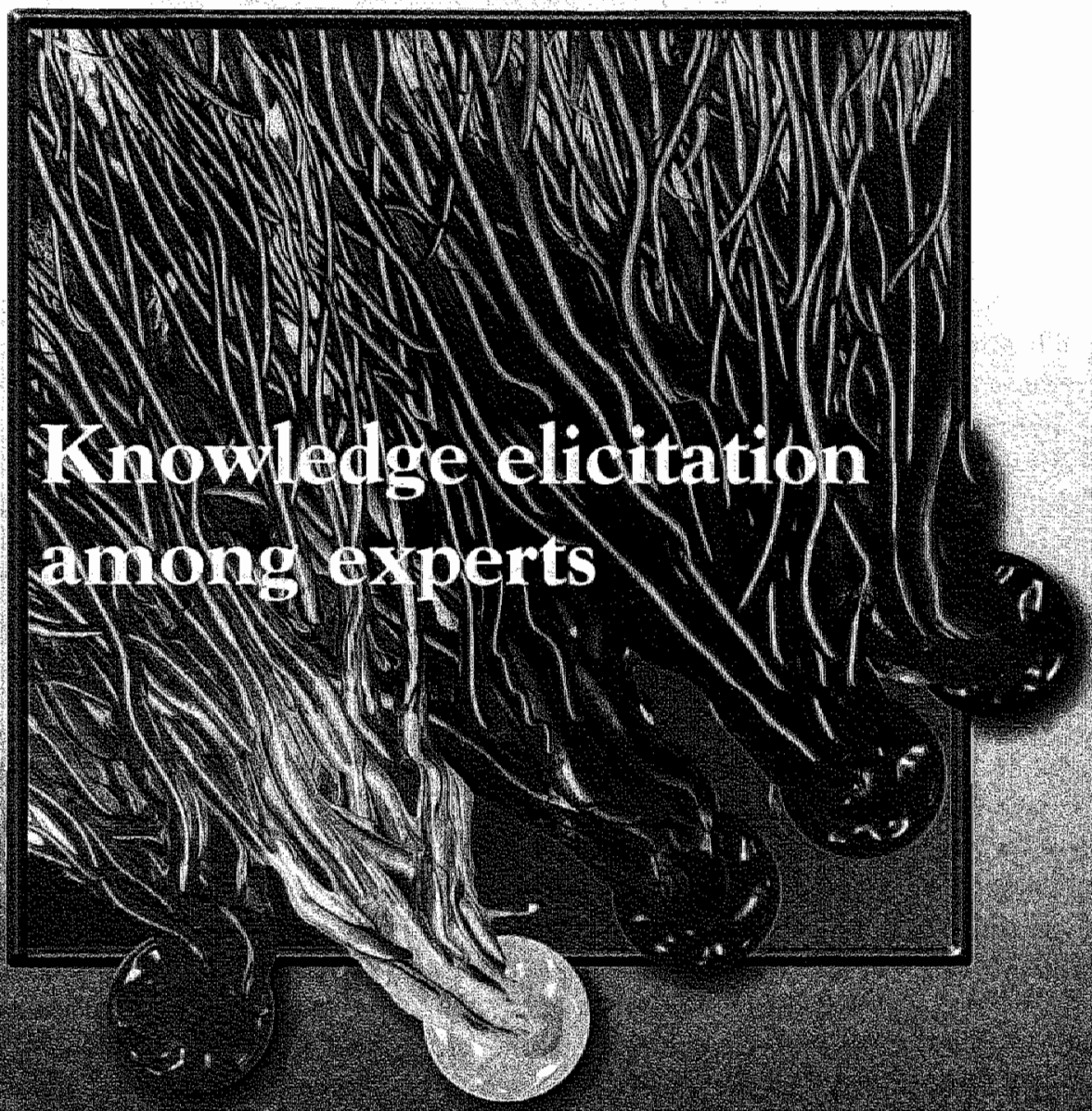

Submitred for publication:

van Ast JE, Renier WO, Talmon J1, Hasman A. Diagnostic Reference Frames for Scizures.

Knowledge elicitation and results. 


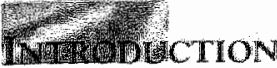

Since the 1960s the International League Against Epilepsy (ILAE) has successively developed several classifications for epileptic seizures $[1,2]$. The ILAE recommends the use of a classification "in the hope that it will bring some measure of unifomity in the use of dianostic terms, facilitate comparison of cases, improve methods of evaluating therapy and eventwally further the understanding of the causes of epileptic seizures ${ }^{\prime 2}$ [1]. To reach these goals, the concepts used in the classification should be clearly defined.

The mostrecent accepted International Classification of Epileptic Seizures (ICES) dates from 1981 121. This classification provides rather general descriptions of the seizure types, which led to variability in its use [3-6]. It has been shown that the use of well defined and clearly agreed upon definitions of seizures reduces the inter-observer variability [4]. Therefore there is a need for comprehensive, (semi-) quantitative and structured descriptions of all seizure types. We call such descriptions Diagnostic Reference Frames (DRFs). These DRFs can serve as a frame of reference in the process of diagnosing patients with epilleptic seizures. We searched for such seizure type descriptions in published resources. The descriptions of the signs and symptoms of different seizure types are scattered over various publications that quite often use a different terminology $[2,7-15]$. Sometimes qualitative terms (e.g. usually, uncommon) are used to indicate the frequency of occurrence of signs and symptoms and sometines quantitative information is given. Because in each qualitative study authors used their own set of concepts to describe the seizure rypes and because in the quantitutive studies different study populations were included on which frequency estimates about the occurrence of signs and symptoms were based, a meta-analysis approach is not feasible. Hence we decided to use the clinical experience of neurologists/ epileptologists to derive DRFs.

From a pilot study we concluded that it is possible to elicir knowledge by using opinions of a group of neurologists/epileptologists for building DRFs [16, 17]. From this feasibility study we learned that the knowledge elicitation procedure could be improved in several walys.

For our pilot study we developed a list of symptoms by extracting them from seizure acserintons whe wature. We further expanded the list based on clinical experience. To

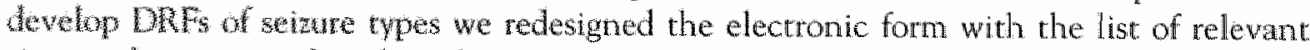

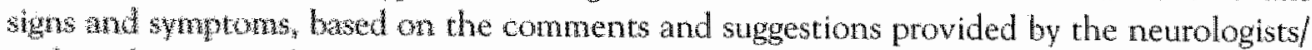
epheptohgists in the plot sudy. This revised list can be considered as a terminology representing the toman of concepts for describing epileptic seizures and is used by us to describe the wrous seizure types. We used the seizure types from the ICES of 1981, because this classification is the most recent accepted one and neurologists are familiar with the temmology. Rather then asking for frequently occurring signs and symptoms as we did in the pilot study we asked to evaluate the frequency of occurrence of each sign and symptom for given seizure type. This gives additional quantitative information that can be made of use in e.g. decision support systems.

In this paper we describe our knowledge elicitation procedure, present the DRFs for different seizure types and report about the initial validation of the DRFs. Signs and symptoms that discriminate between the seizure types are indicated.

In the following sections the term (seizure) manifestation will be used to indicate both signs and symptoms. 


\section{Electronic form}

The refined electronic form consisted of 156 manifestations grouped in eight clusters: seizure characteristics ( 22 items), pre-ictal phenomena ( 4 items), moror signs and symptoms (43 items), non-motor signs ( 8 items), consciousness ( 10 items), somatic reactions ( 17 items), post-ictal phenomena (17 items) and EEG-characteristics ( 35 iterns). See Appendix III for a complete list of the manifestations included.

Neurologists/epileptologists were asked to estimate on a 5-point scale how often manifestations occur for a given seizure type. The users were informed about the meaning of the 5 -point scale: $1=$ nearly not occurring $(<20 \%), 2=$ seldom occurring $(20 \%-40 \%), 3$ $=$ regularly occurring $(40 \%-60 \%), 4=$ frequently occurring $(60 \%-80 \%)$ to $5=$ nearly always occurring $(>80 \%)$. To evaluate our approach we restricted our knowledge acquisition to the most frequently accurring seizure types relevant in clinical practice: simple partial seizures (SPS), complex partial seizures (CPS), myoclonic seizures (MS), tonic-clonic seizures (TCS), typical absence seizures (TAS) and atypical absence seizures (AAS) [17].

\section{Knowledge elicitation process}

Dutch neurologists/epileptologists were asked to estimate the frequency of occurrence of manifestations for the six included seizure types [18]. We calculated the mean and the standard deviation of the responses for each manifestation in each seizure type. In a feedback round the participants were asked to review their responses focusing on manifestations with divergent responses. The feedback was paper-based and consisted of a general part that was the same for all participants and a part related to the responses of the particular participant. The general and individual parts were presented at the same time.

In the general part of the form all participants were asked to review the answers for those manifestations of which the variance of the responses was equal or larger than 2. For these manifestations the distribution of the responses of all participants was presented and the participants were asked to give a new estimate of the frequency of occurrence. Their original responses were not identified on the feedback form. The threshold of 2 was set for practical reasons: on the one hand we wanted all potential diverging responses to be reviewed, but on the other hand the number of manifestations to review needed to be manageable.

In the individual part of the feedback form participants were asked to review their responses for manifestation groups that we suspecred not to be considered by the participant in the first round. The selection criterion was that the values for all manifestations in a manifestation group had the default-value of 'nearly not occurring'. Individual participants were also asked to review those manifestations for which their responses differed at least two points on the 5-point scale from the responses of each of the other participants (but the variance of the total distribution was less than 2). In this case the responses given by that participant and the distribution of the responses of the other participants were presented on the feedback form. Modifications in response could be made on the form and sent back to the researcher (W/A). When a manifestation was not modified during the feedback round, the frequency estimate given in the first round was kept.

To assess the reliability of the averaged frequency estimates we computed the inter-rater intraclass correlation coefficient (ICC) [19]. The ICC is defined as the true variance 
(caused by the differences in frequency of occurrence of the manifestations per seizure type) divided by the total variance (true variance plus the variance caused by random and systematic errors made by the participants). If the $1 \mathrm{CC}$ is equal to or larger than 0.9 (90\% of the total wariance is caused by true differences in the frequency of occurrence of the manifestations) we may conclude that the average frequency estimated for each manifestation within a given seizure type is reliable [20].

Using this interrater $1 C C$ we concluded that the averaged frequency estimates based on the Dutch participants" responses after the feedback round were not reliable enough [21].

The $1 \mathrm{CC}$ increases when the number of observers increases because errors are averaged away to some extent. The Spearman-Brown prophecy formula relates the observed ICC based on a given number of observers to the $1 \mathrm{CC}$ that may be obtained with any other number of observers. This formula allows us to calculate for each seizure type the number of additional participants needed to achieve an ICC of 0.9 [20].

We calculated how many additional participants were needed to achieve reliable averaged frequency estimates and recruited these additional participants at the $5^{\text {th }}$ European Epilepsy Congress. Neurologists/epileptologists were asked to fill out the electronic form for at least one seizure rype rather than all seizure types because of the limited time between the sessions at the congress. After completing the form, these participants were asked to review their responses for the ten manifestations with the largest normalized difference from the mean frequency estimate based on the responses of the Dutch participants. The distribution of the responses of the Dutch participants and the response of that participant were presented on the electronic feedback form. Changes in response could be made on the feedback form and were stored in the database.

Since we used a convenience sample we investigated whether there were neurologists/ epileptologists with diverging opinions abour the frequency of occurrence of manifestations. We identifted only one participant with diverging opin ions for some seizure types [21]. The averaged frequencies of occurrence of manifestations derived from the responses by the Dutch and congress participants for simple partial seizures, complex partial seizures, myoclonic seizures, tonic-clonic seizures and typical absence seizures appeared to be reliable i.e. less than $10 \%$ of the total variance is atributable to random and systematic variations among the participants. For arypical absence seizures a larger number of observers is needed because of the lack of cypical characteristics of thils seizure type [21]. Because we had not chough participants atypical absence seizures were not further analyzed.

\section{Analysis}

Based on the estimates of all participants we calculated the mean frequency of occurrence for each manifestation $s_{j}$ in each seizure type $S_{j}-p\left(s_{j} / S_{j}\right)$ - by recoding the responses of the participants according to the following scheme: $1 \rightarrow 0.10,2 \rightarrow 0.30,3 \rightarrow 0.50,4 \rightarrow 0.70$ and $5 \rightarrow 0.90$ and computing the mean of the recoded results.

Some items like 'duration of seizure' can have a range of values. The range of values was divided into a number of intervals. For each interval the neurologist/epileptologist could enter a frequency of occurrence. For an actual seizure one and only one answer option can be selected. Therefore normalization is necessary. These normalized manifestations are marked with an asterisk in appendix III.

To identify manifestations that discriminate between the seizure rypes we calculated for each manifestation the likelihood ratio between the $p\left(s_{i} / S_{j}\right)$-values of manifestations of all 
possible pairs of seizure types. The likelihood rario is defined as the natio of the probability that a given manifestation $s_{i}$ occurs in a particular seizure type $S_{1}$ and the probability that that same manifestation occurs in another seizure type $S_{k}$ i.e. $p\left(s_{i} / S_{j}\right) / p\left(s_{i} / S_{k}\right)$. We seliscted the 30 highest likelihood ratios and nominated these manifestations as discriminative.

In a previous study we concluded that the frequencies reported here were estimated reliably $[21]$, but we need to assess also whether the frequency estimates for each manifestation are valid. Therefore we determined the validity of the DRF's in two ways. First a face-validity check was performed by one expert (WR) who did not fill out the electronic form, but was involved in the development of the electronic form. A second validity test involved the comparison of our results with the seizure descriptions mentioned in the ICES of 1981 [2]. We searched the text for manifestations with an indication of a frequency of occurrence. We excluded all manifestations of which it was said that they 'may occur' since 'may' hardly provides a quantitative indication.

\section{D.t. The}

\section{Participants}

Forty-one Dutch neurologists/epileptologists were asked to fill out the electronic form. Seventeen neurologists/epileptologists were willing to participate and finally ren neurologists/epileptologists filled out the electronic form for one or more seizure types. Seven of them filled out the electronic form for all seizure types. Eight of them had ten or more years of clinical experience. Eight participants treated on average at least one epilepsy patient per: day. One participant was member of the Task Force on Classification and Terminology of the International League Against Epilepsy (ILAE).

Of the nineteen participants tecruited at the $5^{\text {th }}$ European Epilepsy Congress, five did not completely fill out the electronic form for one seizure type and were excluded from the analysis. Thirteen participants estimated the frequency of occurrence of all manifestations

\section{Dutch participants}

with epilepsy

4 neurologists

6 epileptologists

\section{Congress-participants}

4. neurologists

7 epileptologists

2 neurologist residents

1 paediatrician

\section{Experience in treating patients}

1 of them thas $<10$ years of experience

3 of them thave $>10$ years of experience

10 them has < 10 yeas of experience

5 of them have $>10$ years ol experience

Table 1 . Overview of the qualifications of the participants
Frequency of trating patients with epilepsy

At least one patient per two days

Eeryday

twery day

Every day

Frequency of treating patients with epplepsy

Every day

Every day

Fery day

Every day

At least one patient pert two weeks

At least one patient per three days 
in one seizure type and one participant estimated the frequencies for two seizure types. Twelve participants came from Europe, one from South Africa and one from the United States of America. Thirteen participants were either neurologist, epileptologist or resident in one of the mentioned professions ( 4,6 and 3 , respectively). One paediatric neurologist filled our the electronic form as well. Seven participants had ten or more years of clinical experience. Eleven participants treated on average at least one epilepsy patient per day. None of the participants was member of the ILAE Task Force on Classification and Terminology.

Table 1 gives an overview of the qualifications of the participants. Table 2 gives an overview of the number of neurologists/epileptologists who filled out the electronic form and provided feeclback for each seizure type.

\begin{tabular}{|c|c|c|c|c|c|}
\hline $\begin{array}{l}\text { Sezure } \\
\text { type }\end{array}$ & $\begin{array}{l}\text { Dutch } \\
\text { part }\end{array}$ & $\begin{array}{l}\text { Dutch part } \\
\text { providing feedback }\end{array}$ & $\begin{array}{l}\text { Congress } \\
\text { part }\end{array}$ & $\begin{array}{l}\text { ACongress pait, } \\
\text { providing feed back }\end{array}$ & $\begin{array}{c}\text { Total h part (Dutch } \\
\text { and Congress) }\end{array}$ \\
\hline SPS & 9 & 7 & $4^{*}$ & 1 & 13 \\
\hline CPS & 10 & 7 & $4^{*}$ & 4 & 14 \\
\hline MS & 9 & 7 & 3 & 2 & 2 \\
\hline $\mathrm{TCS}$ & 8 & 6 & 2 & 2 & 10 \\
\hline TAS & 7 & 5 & 0 & $x$ & 7 \\
\hline$A A S$ & 1 & 5 & 2 & 2 & 9 \\
\hline
\end{tabular}

Table 2. Overview of number of neurologists/epileptologists who participated and provided feedback

\section{Mean frequency of occurrence}

The averaged frequencies for the manifestations in each seizure type are presented in appendix III.

To study how sensitive the $p\left(s_{i} / S\right)$-values were for changes in the assignment of probabilities to the codes we used various sets of assigned probabilities (e.g. $1 \rightarrow 0.05,2 \rightarrow$ $0.25,3 \rightarrow 0.50,4 \rightarrow 0.75$ and $5 \rightarrow 0.95$ ) instead of the earlier mentioned probabilities. We made the assignments symmetrical around $50 \%$. The assigned values were restricted to the corresponding interval mentioned on the input form. This resulted in only slightly different $p\left(s_{i} / S_{j}\right)$-values with an absolute maximum change of less than $5 \%$.

\section{Discriminating manifestations}

The 30 manifestations with the highest likelihood ratios (range of 13.36-21.61) were found mostly in the clusters 'consciousness' and 'post-ictal phenomena'. In the cluster 'pre-ictal phenomena' no discriminative manifestations were found. The manifestations that discriminate are presented in Table 3.

Changes in consciousness were the most discriminative manifestations between SPS and all other seizure types except MS. Changes in consciousness are divided in "influence of seizure on ongoing activity", "influence of seizure on response to stimuli" and 'speed of loss of consciousness". In SPS and MS consciousness is not affected by the seizure attack. 


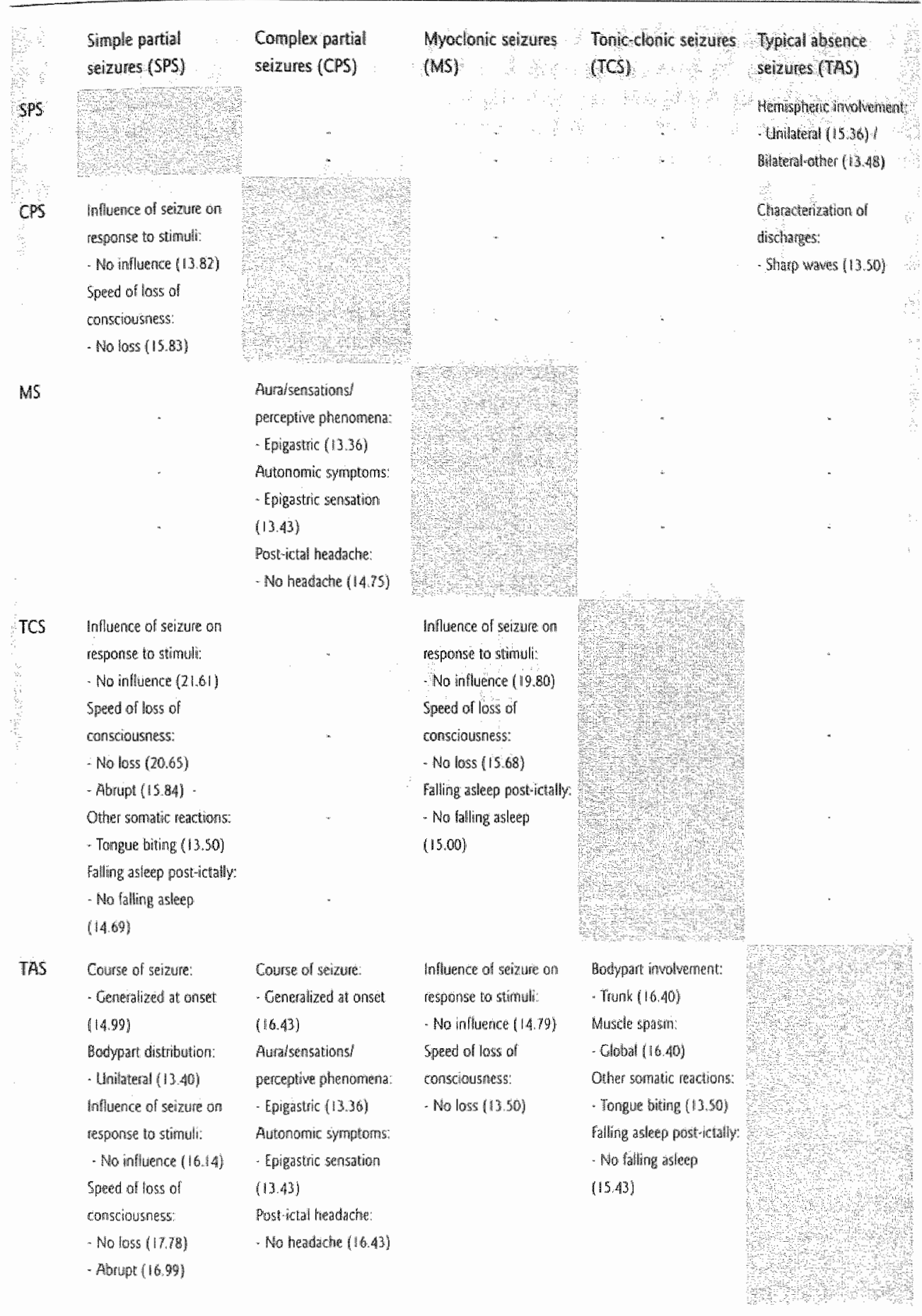

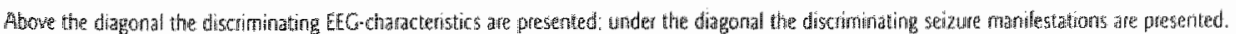

Table 3. Manifestations that discriminate between seizure rypes with the likelihood ratios (in parenthesis) 
Post-ictal phenomena like 'falling asleep' and 'headache' have a high likelihood ratio for CPS and TCS. These phenomena are characteristic for CPS and TCS, but not for the other seizure types. Among the 30 manifestations with the highest likelihood ratios, none discriminated between SPS-MS and CPS-TCS. Berween SPS-MS and CPS.TCS the most discriminative manifestarion was 'generalized at onset' (cluster 'seizure characteristics') with likelihood ratios of 9.89 and 9.78 respectively. Among all likelihood ratios they are ranked as the $63^{\text {rd }}$ and $67^{\text {th }}$ manifestation, respectively.

\section{Validity of the DRFs}

The expert performing the face-validity check concluded that the $p\left(s_{i} / S_{i}\right)$-values of each manifestation for the different seizure types are valid: there were no obviously wrong results.

In the seizure description of the ICES the frequency of occurrence of manifestations is seldom quantified. Of most manifestations it is said that they 'occur' or 'may occur'. A more descriptive indication about the frequency of occurrence is only given sparely and varies from 6 manifestations in MS to 14 manifestations in TAS.

Concepts used to describe the frequency

of occurrence of manifestations

\begin{tabular}{|c|c|c|c|c|c|}
\hline 4 & SPS & CPS & TAS & MS & TCS \\
\hline In the majority of patients & & & & & $0.7773(1)$ \\
\hline Usually & $\begin{array}{c}0.4269 \\
0.7733(4)\end{array}$ & $\begin{array}{c}0.2092 \\
0.7 \| 79(3)\end{array}$ & $\begin{array}{c}0.6039- \\
0.9364(4)\end{array}$ & & $\begin{array}{c}0.5000- \\
0.6950(2)\end{array}$ \\
\hline frequently & $0.3038(1)$ & $\begin{array}{c}0.1378 \\
0.3037(4)\end{array}$ & $\begin{array}{c}0.1071 \\
0.2714(3)\end{array}$ & & $\begin{array}{c}0.5189 \\
0.6000(2)\end{array}$ \\
\hline Often & & & & & $0.5300(1)$ \\
\hline Rarely & $0.0526(1)$ & & $0.0786(1)$ & & \\
\hline Sometines & & & & $\begin{array}{c}0.1625 \\
0.4708(6)\end{array}$ & $\begin{array}{c}0.4600 \\
0.5100(2)\end{array}$ \\
\hline Occasionally & $\begin{array}{c}0.1769 \\
0.3000(2)\end{array}$ & $0.4643(1)$ & $\begin{array}{c}0.0500 \\
0.271 .4(6)\end{array}$ & & \\
\hline In some patients & & & & & $0.4350(1)$ \\
\hline $\begin{array}{l}\text { Number of manifestations described } \\
\text { with a frequency estimate }\end{array}$ & 8 & 8 & 14 & 6 & 9 \\
\hline
\end{tabular}

Table 4. Range of $p(s / S)$-values matched to the concepts used to describe the frequency of occurtence of manifestarions in the $1 \mathrm{CES}|2|$ 
Table 4 shows the various concepts used in the ICES to describe the frequency of occurrence of manifestations as well as the range of $p\left(s_{i} / S_{j}\right)$-walues for these manifestations as obtained in our study. The concepts 'usually' and 'frequently' are used in almost all seizure types to give an indication about the frequency of occurrence of manifestations. We observe that the concept 'usually" has a wide range of $p\left(s / S_{j}\right)$-values in our study. For 'frequently' the range of $p\left(s_{i} / S_{j}\right)$-values is small but relatively low.

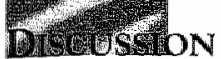

The knowledge elicitation approach used in this study resulted in reliable and face-valid DRFs for simple partial seizures, complex partial seizures, myoclonic seizures, tonic-clonic seizures and typical absence seizures. The DRFs provide quantitative data on the frequency of occurrence of manifestations for various seizure types. These quantitative data can be used in the diagnostic process. We further provide a list of manifestations that discriminate between pairs of seizures, derived from the data in the DRFs.

Although the variance in the responses for some manifestations was large, an ICC of 0.9 is obtained for the different seizure types. This means that most of the variation in the responses due to random and systematic errors by the participants is averaged away. When the responses are to be used for a general description of the DRFs an ICC of 0.7 would have been sufficient. In our study we required an ICC of 0.9 to be sure that the averaged frequency estimates for the manifestations are reliable enough [20,22].

\section{Methodological issues}

In this study we used a convenience sample of neurologists/epileptologists to create DRFs. In a previous study we concluded that the averaged frequencies of occurrence for the manifestations in the DRFs based on the Dutch estimates were not reliable enough. Based on the frequency estimates of the Durch participants we predicted how many congress participants were needed to obtain reliable frequency estimates (ICC of at least 0.9). Adding this number of participants indeed resulted in an ICC of 0.9. This indicated that there were no obvious differences in the opinions about the frequencies of occurrence among the Dutch and the congress participants. We determined for each congress participant whether he/she had deviating opinions as compared with those of the group of the Dutch participants. We also assessed whether each participant of the total group of participants provided frequency estimates consistent with the estimates of all other participants. Based on these results we concluded that the congress participants and the Dutch participants appeared to be samples from the same population [21].

The number of participants needed to obtain reliable DRFs is lower than the number determined from the pilot study [16], a phenomenon we already predicted because of the redesign of the study. In the pilot study participants scored their responses on a binary scale while in this study a 5-point scale was used. To determine whether a 5-point scale indeed would result in a higher reliability we transformed the responses of the participants using the 5 -point scale to a binary scale (responses $1.3 \rightarrow 0$, responses $4.5 \rightarrow 1$ ) and computed the ICCs for the different seizure types. For example, for SPS the ICC based on the 5-point scale was 0.875 and based on a binary scale 0.826 . Lower ICCs were found for binarized responses in all seizure types as compared with the ICCs for 5-point scale responses. The frequencies apparently can be better estimared on a 5-point scale. A 
second factor that could be of influence is the redesign of the form such that manifestations were now presented in clinically meaningful clusters rather than in alphabetical order. This could have had a positive effect on the random error in the responses of the participants.

\section{Validity of the DRFs}

The DRFs and the manifestations that discriminate between the seizure types are face-valid.

We compared the descriptive frequency expressions in the ICES with the $p\left(s / S_{j}\right)$-values we found. Only for a limited number of manifestations an indication of the frequency of occurrence is given. If an indication is given, the concept may have different meanings to different people, which can lead to misunderstandings. In this study the $p\left(s_{\mathrm{p}} / S_{j}\right)$-values corresponding to the descriptive expressions in the ICES showed a large range which indicates the different interpretations of qualitative expressions. This phenomenon was also observed by Kong et al. [23].

We also searched - but not systematically - for studies that report quantitative results for the frequency of occurrence of manifestations $[7,14,24]$. We compared these frequency rates to our $p\left(s_{i} / S_{j}\right)$-values and found large differences. These differences may result from the fact that we asked neurologists/epileptologists to estimate the frequency of occurrence for e.g. complex partial seizures without specifying from which patt of the brain the seizure is originating. In one of the quantitative studies, the authors selected patients with complex partial seizures originating from the frontal and mesial temporal lobe [24]. Because few of the manifestations we used are identical to the manifestations reported in these studies the frequency values could not be compared systemarically.

The conclusion that consciousness discriminates between simple and complex partial seizures seems trivial. According to the ICES the impairment of consciousness is an important manifestation that differentiates between SPS and CPS. Finding such trivial discriminating features does not prove that our study is valid, but missing them would have shown that our method is a failure.

The complex partial seizures include seizures with very different semiology. The subcategories of CPS refer to the location of the seizure onset zone which is important for prewsurgical assessment. In clinical practice medical treatment is commonly considered first. Our goal is to assist physicians in a systematic registration of manifestations and diagnosing the seizure type to decide on the initial treatment. For CPS there is one preferred antiepileptic drug treatment irrespective of the localization of the seizure onset and therefore we grouped all these seizures in a single class.

Most of the variance is explained by the variance due to the differences in the frequency of occurrence of the manifestations. An increase of the number of participants does increase the reliability of the frequency estimares but it does not necessarily entail that all frequency estimates are accurate. The frequency estimates can be reliable but the usability of these estimates depends on how good the neurologists/epileptologists are in estimating the frequency of occurrence of the manifestations.

To determine the accuracy of the responses an observational study in a clinical setting is needed. Such a study would require, however, that clear definitions of the seizure types exist and that high quality observations are being made. In an observational study we assessed whether medical students and neurology residents are accurate in reporting manifestations they observed on video recordings. We concluded that these participants incompletely 
reported seizure manifestations. They also reported manifestations that were not visible on the video recordings. This indicates that assessing the accuracy of the results of our study is not an easy task [21].

\section{Potential use of DRFs}

In the introduction we mentioned that the DRFs could serve as a frame of reference in the process of diagnosing patients with epileptic seizures. There are several clinical and scientific advantages in using DRFs.

A DRF represents the manifestations of a specific seizure type. The DRF can serve as a guideline for acquisition and documentation of patient data. Based on the manifestations specified in the DRFs, forms may be designed for a structured approach to collect patient data. This may increase completeness and uniformity of patient information $[5,25]$ needed for correcrly classifying seizures, starting therapy and providing information. The DRFś can also be used as an auditing tool by the physician to check whether the patient's manifestations are in agreement with the manifestations listed in the DRF for the seizure type that he/she tentatively diagnosed.

From a scientific point of view the univocal use of terminology for recording patient data and classifying seizure types enhances scientific exchange and discussion about optional treatment strategies. Also incidence and prevalence rates of seizure types can be compared between regions because the seizure types are classified based on the same terminology and criteria.

For knowledge elicitation a list of all possible manifestations is needed but for manual usage in a clinical setting it is cumbersome. Manifestations with almost equal $p\left(s_{i} / S\right)$-values for all seizure types could be left out for clinical use. However, for knowledge elicitation of other seizure types all manifestations need to be included to prevent that manifestations that appear to have discriminative power are not included in a DRF:

To test the clinical use of the DRFs we implemented the DRFs in a stand-alone decision support system based on Bayes' rule. In this system the following functions were implemented:

- Observed patient's manifestations can be registered in a systematic way. When observed manifestations fall in different clusters, one can easily switch from one cluster to another cluster and register relevant manifestations. Registration of the observed mianifestations only will suffice. The manifestations in the decision support system are identical to the list of manifestations presented in the DRFs. As soon as a manifestation is registered, the $p\left(s_{i} / S_{j}\right)$-values of these manifestations are used to update the probabilities of the different seizure types.

- The DRFs can also be used to generate the most informative manifestations that potentially discriminate between competing diagnoses taking into account the information gathered so far during the consultation. This results in a checklist, limits the number of relevant manifestations and may increase completeness of the registration of patient's manifestations in the (electronic) patient record $[5,25]$.

In Box 1 we present a patient case and explain how the system works. Preliminary results on 18 cases with different seizure types show good agreement between the classification of the system and the neurologist's diagnosis. This indicates that the $p\left(s_{k} / S_{3}\right)$-values are valid and reflect reality.

The inclusion of EEG-characteristics in the DRFs is questionable for several reasons. In our pilot study we concluded that the description of the EEG was too detailed and should 
In this tase fimale patient dewcribed her symptoms occurring dung a seizure. The physician diagnosed that she is sulfering rom bypical abserice serzunes.

SPS CPS MS TCS TAS

\begin{tabular}{|c|c|c|c|c|c|c|}
\hline \multicolumn{7}{|l|}{ Standard questions } \\
\hline Onset of seizure & Sudden: & 01385 & 04391 & 00636 & 0.493 & 01633 \\
\hline Duration of seizure & 40 seconds & 0.1373 & 0.0843 & 02292 & 01727 & 03765 \\
\hline Mowements clonlic & $\mathrm{Mo}$ & 0.0867 & 0.1067 & 0.2817 & 0.0271 & 0.4977 \\
\hline Movernents: tonic & Wo & 0.0646 & 0.0673 & 0.3289 & 0.0039 & 0.5353 \\
\hline Movenentes atonic & No & 0.0568 & 0.0566 & 0.3239 & 0.0036 & 0.5591 \\
\hline Wovements, nifyoctoricic & $\mathrm{MO}$ & 0.0762 & 0.0775 & 0.0495 & 0.0057 & 0.7911 \\
\hline $\begin{array}{l}\text { Conseiousness } \\
\text { Infuntuce of suzure } \\
\text { on ongoing activity }\end{array}$ & $\begin{array}{l}\text { Stops normat } \\
\text { ongoing actiwly }\end{array}$ & 0,0163 & 0.0640 & a.0173 & 0.0068 & 0.8956 \\
\hline $\begin{array}{l}\text { Confisciousmess: } \\
\text { Unfluence of seizure } \\
\text { on response to stimuli }\end{array}$ & $\begin{array}{l}\text { Untesponstwe to } \\
\text { paintul stimuli }\end{array}$ & 0.0026 & 0.0564 & 0.0053 & 0.0131 & 0.9225 \\
\hline \multicolumn{7}{|l|}{ Advice mfunction } \\
\hline Post-ictal hedache & No & 0.0019 & 0.0030 & 0.005 & 0.0018 & 0.9882 \\
\hline $\begin{array}{l}\text { Speed of loss } \\
\text { of constiolisness }\end{array}$ & \multicolumn{5}{|c|}{ of eonstiolusness } & 0.9957 \\
\hline Aurthis & Mo & 0.0001 & 0.0005 & 0.0004 & 0.0002 & 0.9988 \\
\hline Course of the seirure & Disabled & 0.0001 & 0.0005 & 0.0004 & 0.0002 & 0.9988 \\
\hline Hands & No & 0,0000 & 0.0002 & 0.0002 & 0.0000 & 0.9995 \\
\hline Muscle contractions: & Disibiled & 0.0000 & 0.0002 & 0.0002 & 0.0000 & 0.9995 \\
\hline \multicolumn{7}{|l|}{ Symametric/Asymmetric } \\
\hline Abnormal eye movements & Staning ahead & 0.0000 & 0.0001 & 0.0000 & 0.0000 & 0.9998 \\
\hline Confusion & Yes & 0.0000 & 0.0009 & 0.000 & 0.0001 & 0.9989 \\
\hline Epigastric & $\mathrm{Mg}$ & 0.0000 & 0.0003 & $0.000:$ & 0.0000 & 0.9996 \\
\hline $\begin{array}{l}\text { Falling asleep } \\
\text { poskictally }\end{array}$ & 5 leeps $>30$ minutes & 0.0000 & 0.0021 & 0.0002 & 0.0004 & 0.9974 \\
\hline \multicolumn{7}{|l|}{ Additional symplons } \\
\hline Cess sation of sutizure & Sudden & 0.0000 & 0.0005 & 0.0002 & 0.0002 & 0.5992 \\
\hline Tine ol day selzure orcurs & Irfegular & 000000 & 0.0010 & 0.0002 & 00002 & 0.9086 \\
\hline Unaware of serzuro & Yes & 0.0000 & 0.0007 & 0.0001 & 0.0001 & 0.9991 \\
\hline Speech disturbance & yes & 0.0000 & 0.0050 & 0.0001 & 0.0005 & 0.9945 \\
\hline
\end{tabular}

hach time a sign or symptomi is registered as preseitu of absent; the probabities for the warous seizure types are talculated. The fistsil-wallues are used to undate the probabilities of the different seizure types.

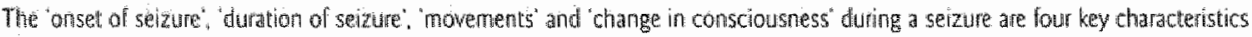
that are asked to every patient or relative in the process of diagnosing seizures. Based on the answers gathered after these lou? cliarateristics the application determines a symptom with the bighest distrminating power beween the competing diagnoses (adve -function) that is then asked to the patient or relatiwe. This procedure is followed 10 times. Frally the patient has the opportunity to report symproms that were mot discussed yet ("additional symptorns').

In this patient we observe that ater the lour key chatacteristes TAS has the highest probability. After registering the answers wo the atvice" and "addational" symptons the probability for TAS increases. After all symptoms are registered the application conciliuded With the probability that the patsent is suffering from TaS.

Box 1. Partient case 
be replaced by more general terms [17]. In this study we used more general terms but it still remains difficult to describe the EEG-characteristics in a sufficient and meaningful way. We found also very few EEG-characteristics that discriminate between seizure types. Furthermore early in the process of diagnosing seizures EEG-data are often not available. Hence it seems advisable not to use this part of the DRF in clinical applications.

Before the DRFs can be used in clinical practice to support physicians in diagnosing seizures all seizure types need to be described by a panel of neurologists/epileptologists using the same material and methods as described in this study. For AAS we concluded that we need more neurologists/epileptologists to obtain a reliable DRF, which is due to the lack of typical characteristics of this seizure type.

\section{Relation to the proposed diagnostic scheme of the ILAE}

In 1993 Lüders discussed the ICES and proposed to develop a seizure classification which identifies seizures based exclusively on ictal seizure semiology [26]. The semiological seizure classification (SSC) is a classification based on the clinical manifestations of seizures and does not include EEG-criteria or other test results $[27,28]$. The SSC permits a precise localization of the ictal onset of seizures which is relevant in pre-surgical evaluation [29,30]. The diagnostic (sub) categories in the SSC can to a large extent be mapped on the manifestations or the clusters of manifestations in our list.

However, Engel [30] calls the SSC a descriptive terminology for ictal events. He also stated that the Task Force on Classificarion and Terminology of the ILAE was planning to create such a descriptive terminology and three new classifications.

This Task Force presented "A proposed diagnostic scheme for people with epileptic seizures and with epilepsy" in 2001 [31]. It consists of five axes of which the first two axes are relevant in the context of our study. The first axis contains the "Glossary of descriptive terminology for ictal semiology" [32] and the second axis contains a list of epileptic seizure types [31]. The proposal is not primarily a classification but a diagnostic scheme for bedside usage [33].

This ILAE proposal provides the opportunity to practitioners to use the standard terminology from axis 1 to communicate what is observed and what a patient reports about a seizure. With the Glossary (axis 1) the ictal events of epileptic seizures can be described to the degree of detail required for clinical or research purposes [32].

On the website of the ILAE Task Force on Classification and Terminology (www.epilepsy.org) several seizure types of axis 2 are described but in these descriptions the standard terminology from the Glossary is not (yet) used systematically.

Our list of terms can to a large extent be mapped on the Glossary of the proposed diagnostic scheme. The manifestations in the clusters 'non-motor', 'autonomic events", 'duration', 'prodrome', 'post-ictal phenomena', 'motor' and 'somatotopic modifiers' of the Glossary are also included in our list of manifestations. These clusters broadly cover the sarne concepts although sometimes different terms are used (e.g. in the Glossary 'Todd's phenomenon' is mentioned; in our list of manifestations we use 'paresis' and 'paralysis'). We included the clusters 'seizure characteristics' and "EEG-characteristics" which are not present. in the Glossary. The concepts under the cluster 'modifiers and descriptors of seizure timing' in the Glossary cannot be mapped to one of the clusters in our list of manifestations. The Glossary uses the concept 'dyscognitive' to indicate a disturbance of cognition, while on our 
list of manifestations the concept 'consciousness' is used which refers to the degree of awareness and/or responsiveness of the patient to externally applied stimuli.

Our results for MS, TCS and TAS can easily be incorporated as these seizure types are defined in the proposal in a way similar to the ICES of 1981 . The results for SPS and CPS are more difficult to incorporate since these terms are not included in the new proposal anymore. Descriptions of the newly defined seizure rypes that are subtypes of SPS and CPS in the old classification are unforturiately lacking. Hence the value of our results for SPS and CPS in relarion to the new classification cannot be judged yet.

Our study showed that for well known seizure types it is possible to derive reliable frequency estimates for the various manifestations. However, when new seizure types are proposed, as is done in the second axis of the proposal, more participants may be needed to derive relialsle DRFs. Epileptologists may lack experience with these concepts to provide reliable frequency estimates for the various manifestations.

\section{Carisions}

We have presented the frequencies of occurrence of manifestations in a number of seizure types. This type of information is not available in literature.

Our study indicates that the DRFs we developed are relable and face-valid for simple partial seizures, complex partial seizures, myoclonic seizures, tonic-clonic seizures and typical absence seizures. The method we used provides the opportunity to obtain quantitative information on manifestation occurrences for the most common occurring seizure types. For less specific seizures like atypical absence seizures more experts are needed to obtain reliable descriptions, which is probably due to the variability in expression of this type of seizure. 


\section{ThITIRALES}

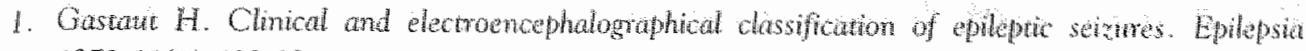
$1970 ; 11(1): 102-13$.

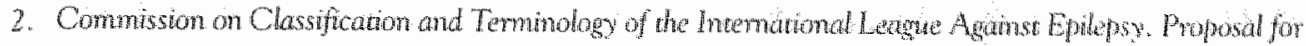

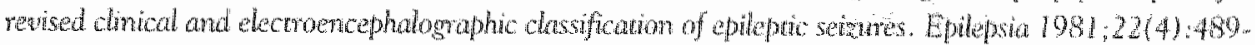
501 .

3. Bodenstemer JB, Brownsumh RD. Knapik JR. Kanter MC, Cowan LD, Leviton A Imerobserver

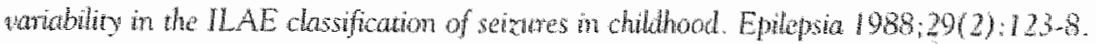

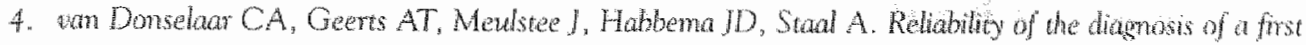
seirux. Nentology 1989,39(2 P ( ) :267.71

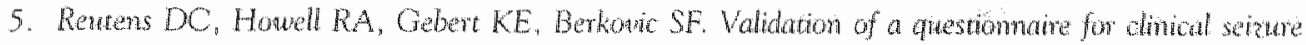
diagnosis. Epilepsia 1992;33(6):1065-71.

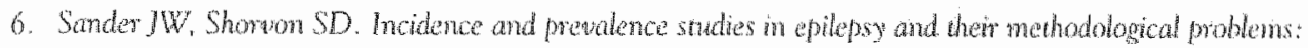
a review. J Nemol Netwosurg Psychiary 1987.50(7):829-39.

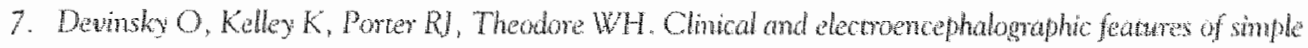
partial seizures. Neurology 1988;38(9):1347-52.

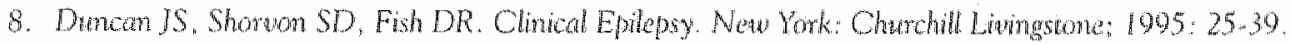

9. Gram L. Eptheptic seizures and syndromes. The Lancer 1990,336(8708):161-3.

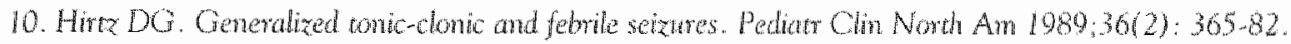

11. Lockman LA. Absence seizures and vatants. Newol Clin 1985,3(1):19.29.

12. Mosewich RK. So EL. A clincal approach to the classificanon of seizues and eplepto syndrones. Mayo Clim Proc 1996:71(4):405-14

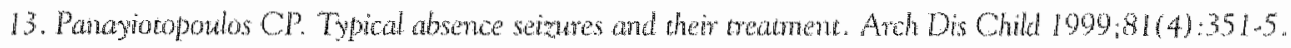

14. Theadore WH, Poter RI, Penry JK. Complex partical serwes: dinal characteristos and differential diagnosis. Neurology 1983;33(9):1115-21.

15. Wyllie E, Rother AD, Luders H. Parial seizures in children: dimical features, medical tratment, and surgical considerations. Pedatr Clin Now A.m 1989;36(2):343-64.

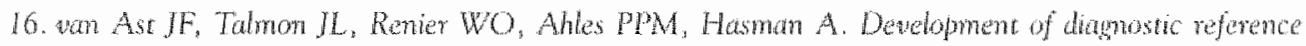

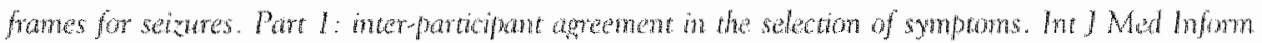
$2003 a ; 70(2-3): 285-292$

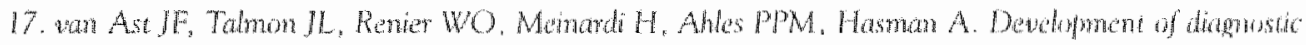

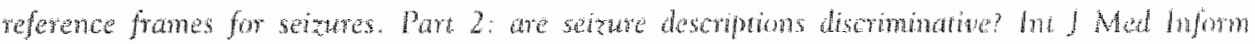
$2003 b ; 70(2-3): 293-300$

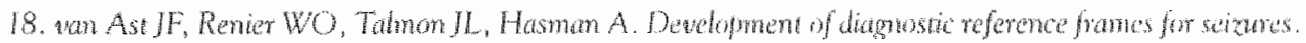
Eptlepsia 2002:43(Suppl. 8):63.

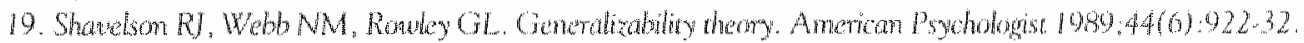

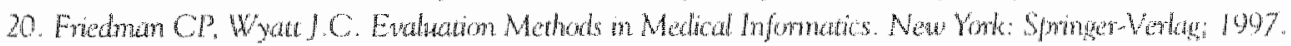

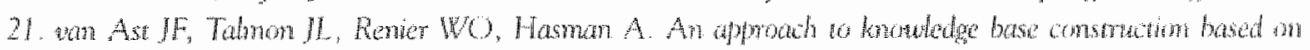
exper opmions. Mediods ho Med 2004,43:427,32.

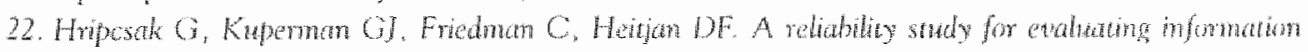

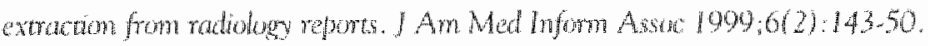

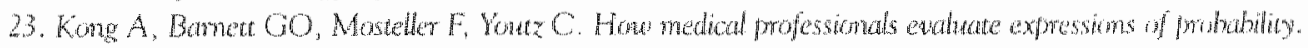
NEngl Med 1986;315(12):7404,

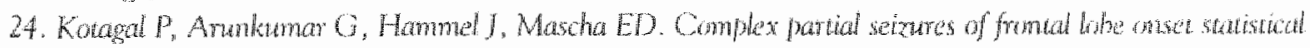
andysis of ictal semiology. Sazme 2003:12(5):268-81 


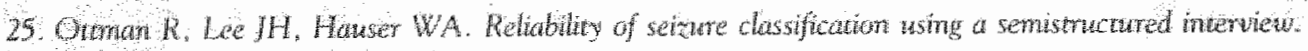
Menolog $1993 ; 43(12) 2526-30$.

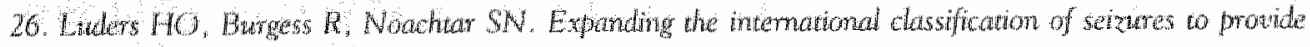

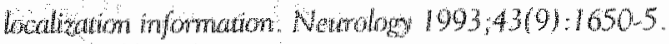

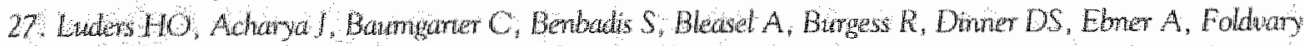

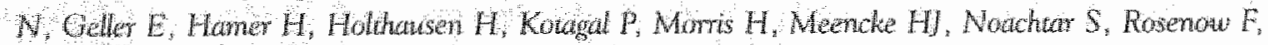

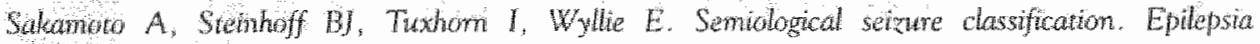
$1098,39(9): 1006-13$

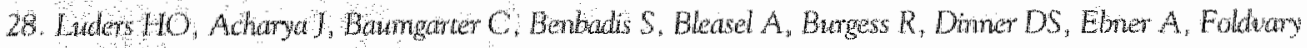
W, Galler E, Homer H. Holhasen H, Kotagal P. Momis H. Meencke H. Noachar S. Rosenow P,

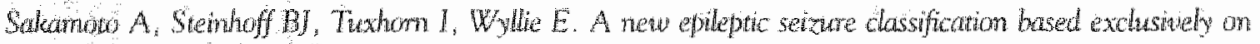
ical senology. Actu Newol Scand 1999,99(3):137-41.

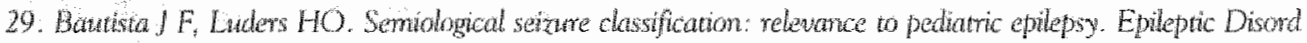
$20002(1) .65-72$; discussion 73 .

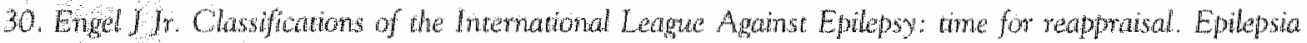
$1998 ; 39(9): 10147$.

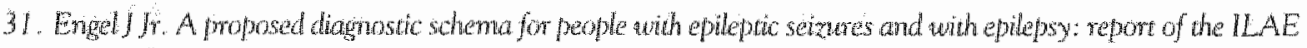
Takk Force on Classificanion and Temonology. Epilepsia $2001,42(6): 796803$.

32. Bhme WT, Luder HO, Mirah E, Tassinari C, Var Emde Boas W. Engel J Jr. Glossary of descriptie taminology for intal semiolagy: repont of the MLAE task force on classification and terminology. Epilepsia $2001: 42(9): 12128$

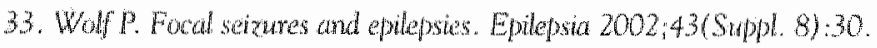




\section{-}




\section{Chapter 6}

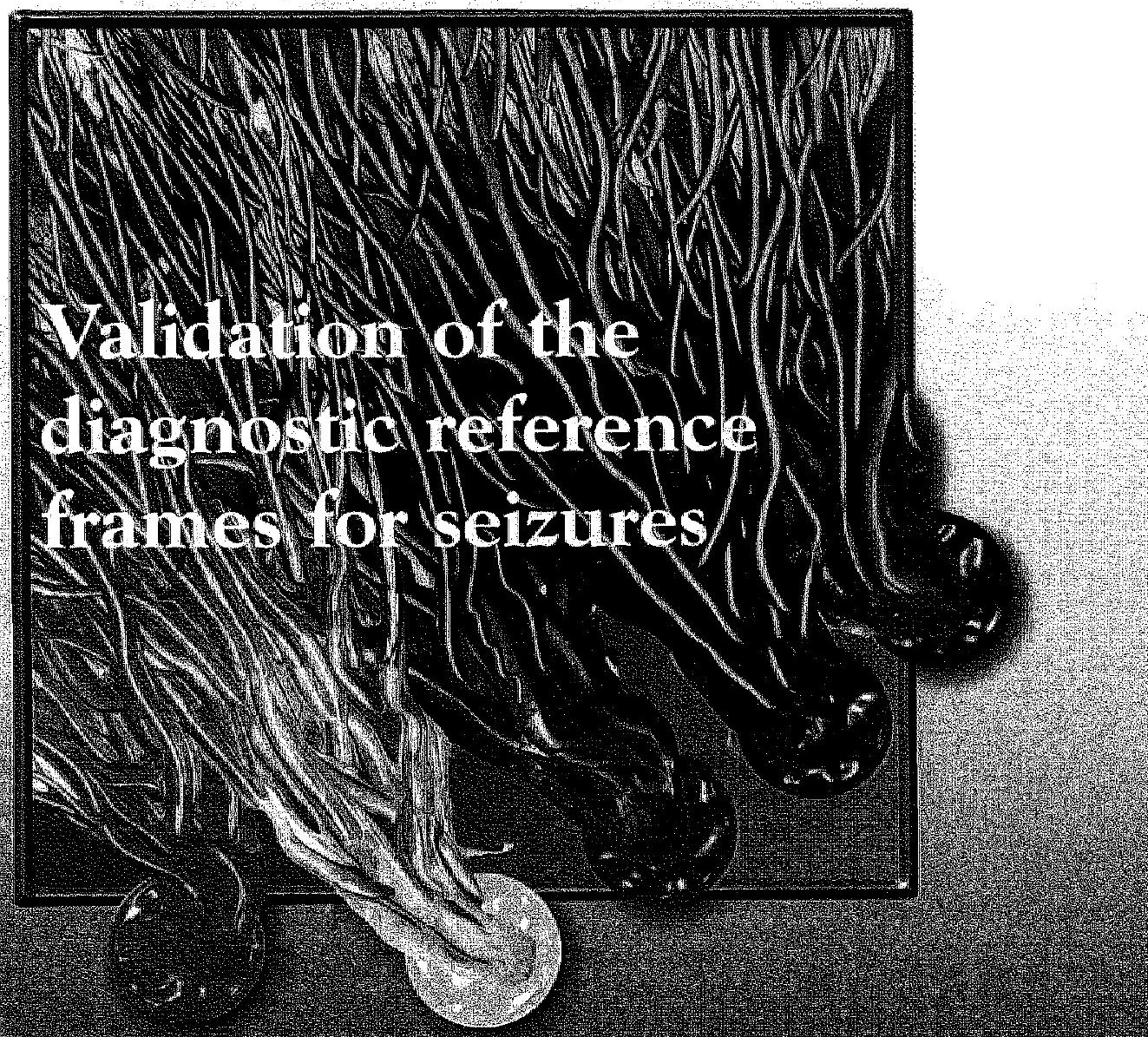

Accepted for publication in Journal of Neurology?

yan Ast IF Renier WO, Talmen IL, Roos JMA, Hasman A.

Diagnosric Reference Frames for Seizures: A validation sindy. 
Since the 1960 s the International League Against Epilepsy (ILAE) has successfully developed several classification systems for epileptic seizures [1, 2]. The most recent accepted International Classification of Epileptic Seizures (ICES) dates from 1981 [1]. This classification provides rather general descriptions of the seizure types, which has led to variability in its use $[3-6]$. It has been shown that the use of well defined and clearly agreed upon definitions of seizures reduces the inter-observer variability [4].

We developed structured descriptions of epileptic seizures, called Diagnostic Reference Frames - DRFs-by us. A DRF consists of a list of manifestations. For each manifestation a conditional probability is given. $A$ high probability $\mathrm{g} 0.9$ indicates that a manifestation is frequently occurring. A low probability e.g. 0.05 indicates that a manifestation is seldom occurring in the seizure type. The same list of manifestations is used across all included seimure types. Neurologists/epileptologists provided estimates of these frequencies of occurrence on a 5 -point scalle from which the probabilities for the DRFs were derived. DRFs were developed for the most common seizure types: simple partial seizures, complex partial seizures, myoclonic seizures, tonic-clonic seizures and typical absence seizures. A DRF can serve as a frame of reference in the process of classifying patients with epileptic seizures.

The knowledge included in the DRFs needs to be verified and validated before it can be used in routine clinical practice. Verification refers to the internal consistency of the data. It can be performed without test cases [7]. It has already been shown, using the intraclass correlation coefficient (ICC) that the estimated frequencies by the experts for each seizure type are consistent 18] and that the DRFs are face-valid.

Validation is the "act of comparing properties of an object with the stated goal as a frame of reference concluding on degree of fulfillment" [9]. In our case the validation of the DRFs is performed by comparing the classifications of a decision support system based on the DRFs with the classification of experienced epileptologists using a series of test cases with known epileptic seizures.

This article describes the results of our validation study. In the following section we first introduce the decision support system in more detal. Next we describe the study design and the results. In the discussion we reflect on the observed accuracy of the DSS and the limitations of our study and the used DSS.

In the following sections the term (seizure) manifestations will be used to inclicate both signs and symptoms.

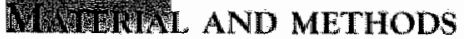

\section{Material}

\section{Dhagnostic seizure support}

As mentioned in the introduction we developed a decision support system, which we call Support in Classification of Seizures (SICS). SICS is a stand-alone system that provides assistance in classifying patients with epileptic seizures. The current system is discriminating between five seizure types: simple partial seizures (SPS), complex partial seizures (CPS), typical absence seizures (TAS), myoclonic seizures (MS) or ronic-clonic Seizures (TCS). 
In SICS the user fills out the administrative parient data: name, gender, date of birth and consultation date. Then the seizure manifestations are presented to the user in seven clusters: 'seizure characteristics', "pre-ictal phenomena', 'motor signs and symptoms', 'nonmotor symptoms', 'consciousness', 'somatic reactions', and 'post-ictal phenomena'. The user can report the manifestations in the order he/she wants.

The role of SICS is to provide a classification of a seizure based on the patient's manifestations. The system uses Bayes' rule to compute posterior probabilities for the included seizure types based on the a priori probabilities and the conditional probabilities for the manifestations reported. We used the normalized incidence rates of the seizure types as a priori probabilities. The system assumes independence among the manifestations. For each seizure type the system provides a probability and the seizure type with the highest probability is considered to be the correct classification.

Each time new information is entered the system updates the posterior probabilities for the different seizure types using Bayes' rule. If the patient does not know the value of a manifestation, the user can report this manifestation as 'unknown' and the posterior probabilities are not updared.

There is also a function available that determines the most informative manifestation that, on average, changes the probability distribution over the included seizure types most taking the information gathered so far into account (advice-function). This allows a sequential elicitation of the most informative manifestations $[10,1]]$. Further the user has the possibility to examine and print the reported manifestarions.

\section{Protocol for systematic registration of manifestations in SICS}

For a systematic registration of seizure manifestations we developed a protocol. After the registration of the patient data the user reponts the status of four key manifestations: 'onset of seizure', 'duration of seizure', 'movements' and 'consciousness' (step 1). Then the system is asked to generate the most informative manifestation and the user reports the answer of the patient or his/her relative(s) for this manifestation. Then the next informative manifestation is determined and presented to the user. Ten times in a row the most informative manifestation is determined and the value entered into the system (step 2). Finally the user can enter manifestations mentioned by the patient or his/her relative(s) and not suggested by the system in the order he/she wants (step 3) as to have a more complete registration of the seizure.

\section{Patient cases}

The patients were selected by three experienced epileptologists from their own patient population.

To include the patients the following criteria were used: 1) the patient was at least 12 years old; 2) the patient was still under treatment of the epileptologists; 3) rhe epileptologist diagnosed the seizure type and 4) the patient was willing to cooperate.

When a patient was willing to participate the researcher (WvA) interviewed the patient or his/her relative(s) about the manifestations occurring during a seizure following the protocol described in section 2.1.2. This interview took place directly after the consultation with the epileptologist or at a later time by telephone. The researcher was blinded for the diagnosis of the patient prior to the interview except for the MS cases. One of the epileptologists was asked to provide these cases and only such cases as to increase the number of cases with MS in our test ser. 


\section{Methods}

To determine the accuracy of the classification of the system the seizure type with the highest posterior probability was compared with the seizure type diagnosed by the epileptologist who treated the patient. The diagnosis made by the epileptologist was assumed to be correct and serves as a reference standard for the classifications provided by the swstem.

Further we assessed how the posterior probabilities changed after each step in the protocol.

When the system's classification differed from the reference standard the patient case was evaluated by one of the epileptologists (WR). The manifestations reported for these cases were presented to the epileptologist. He was asked to give his claissification based on the reported manifestations.

\section{Misulits:}

\section{Description of patient cases}

We included sixty-six patients. The patients and/or their relatives described the manifestations occurring during a seizure. Table 1 gives an overview of how many patients were included for each seizure type.

\begin{tabular}{|c|c|c|c|c|}
\hline $\begin{array}{l}\text { Total of of } \\
\text { patients included }\end{array}$ & manifestations & $\begin{array}{l}\text { Informative } \\
\text { manifestations }\end{array}$ & $\begin{array}{l}\text { Additional } \\
\text { manifestations }\end{array}$ & $\begin{array}{l}\text { Hof wrong } \\
\text { diagnosis }\end{array}$ \\
\hline 1 & $3(43 \%)$ & $5(710 \%)$ & $5(7,9)$ & 2 \\
\hline 10 & $4(40 \%)$ & $4(40 \%)^{2}$ & $9(90 \%)$ & 1 \\
\hline 15 & $9(60 \%)$ & $12(80 \%)$ & $B(87 \%)$ & 2 \\
\hline 28 & $25(89 \%)$ & $28(100 \%)$ & $28(100 \%)$ & 0 \\
\hline 6 & $5(83 \%)$ & $5(83 \%)$ & $5(83 \%)$ & 1 \\
\hline atal & $46(70 \%)$ & $54(82 \%)$ & $60(91 \%)$ & 6 \\
\hline
\end{tabular}

Trible 1. Number of correctly classified seizure types after reporting key, most informative and additional manifestations

\section{Accuracy of SICS}

Sixty of the patient cases $(91 \%, 95 \%$ confidence interval: $84 \%-98 \%)$ were correctly classified by the system after the registration of all elicited manifestarions (Table 1). In 50 of the 60 correctly classified cases the posterior probability for the correct seizure type was 0.90 or higher (Table 2).

For six cases the system did not provide the correct classification. Based on the manifestations reported by the patients or his/her relatives the system provided another classification than the epileptologists. One epileptologist evaluated these six patient cases as well as one case that was correcrly classified by the system. Based on the reported 
manifestations for these seven cases the epileptologist classified the same seizure type as the system in 5 cases. For the case which was correctly classified by the system, the epileptologist also provided the correct seizure type:

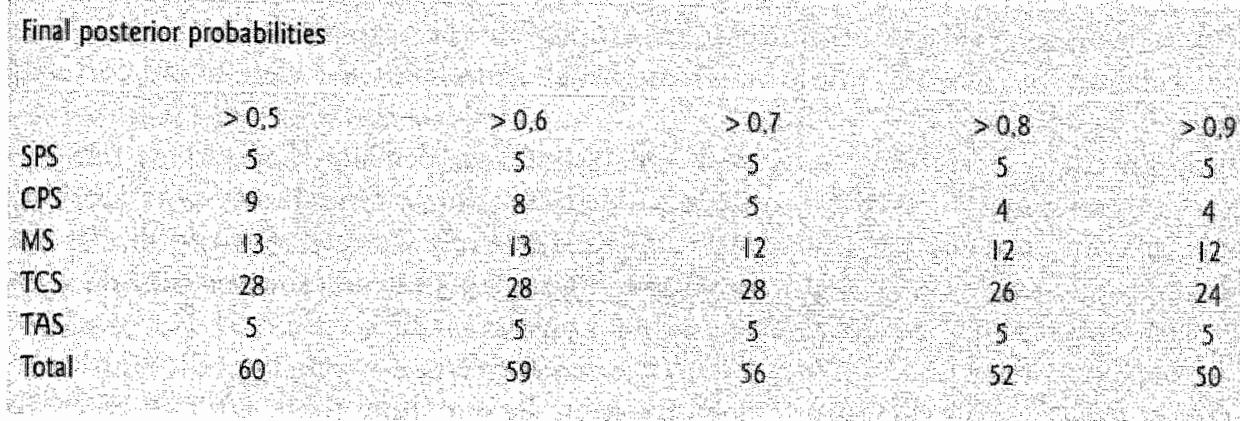

Table 2. Distriburion of final posterior probabilities for seizure types correctly classified by SICS

\section{Eqaluation of the protocol}

Based on the four key manifestations, $46(70 \%)$ of the cases were classified correctly. By asking the ten most informative manifestations 8 more cases ( $82 \%$ ) were correctly classified (Table 1). Reporting additional manifestations resulted in an further increase in the number of correctly classified seizures: one MS case and five CPS cases. For TAS the number of correctly classified seizures did not change when manifestations additional to the key manifestations were reported.

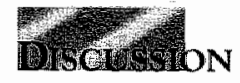

\section{Study design}

We validated the DRFs in an experimental setring. The patient was asked to tell his/her seizure manifestations to the researcher after the consultation with the treating physictan. This does not reflect the normal consultation setting, but more closely resembles the situation in which a (specialist) nurse interviews the patient to elicit relevant clinical information before seeing the physician. The experimental setting in which the researcher is blinded for the classification of the seizure allows us to determine the accuracy of the system under optimal conditions (efficacy test) using the classification of the epileptologists as a reference [12].

Case selection was limited to two locations: an academic neurology department and a specialized epilepsy center. Due to logistic reasons, neurologists see their patients only on a specific day in the week and patients only visit the epileptologist once or twice a year, data collection rook nearly one year. Including a larger population would have required a larger number of participating centers.

\section{Accuracy of SICS}

The performance of the system was $91 \%$. Compared to other decision support systems in neurology the accuracy of SICS is good, even taking into account the relatively wide $95 \%$ 
confidence interval due to the small number of cases. In Epilepsy Expert, a decision support system based on the International Classification of Epilepsies and Epileptic Syndromes [13], 18 of the $25(72 \%)$ patient cases were correctly diagnosed when the diagnosis was based on clinical manifestations solely [14]. Diagnoses given by MICROSTROKE, an expert system for stroke type diagnosis based on clinical information, have been tested for conformity with the final diagnosis of 250 cases in the Hamburg Stroke Data Bank and were found correctly in $72,8 \%$ of the 250 cases [15].

Although SICS performs better then the other two decision support systems in neurology it is not yet proven that an accuracy of $91 \%$ is sufficient for clinical practice. One must also take into account the therapeutic consequences of a wrong classification. For the seizure types included in our system two groups of seizure types can be distinguished each treated with a different therapy. The first group consists of SPS, CPS and TCS and the second group consists of TAS and MS. When the classification is wrong but falls within the same group, there are no therapeutic consequences. In our study there are two cases for which the classification of the system and the correct diagnosis fall in a different therapeutic group. This indicates that only for those two cases the classification would have therapeutic consequences.

In daily pracrice the physician also is not always making a final classification of the seizure during the first consultation. An evaluation of the manifestations and further diagnosis during the next consultations is always needed.

\section{Evaluation of the protocol}

Table 1 shows that the accuracy of SICS increased when informative manifestations are reported. Reporting additional manifestations seem to add less to the accuracy of SICS. This indicates that when key and informative manifestations are reported completely additional manifestations do not have to be reported any more. Only for CPS reporting additional manifestations seemed necessary. The results reported in this paper are based on a reanalysis of the data with the most recent version of SICS. We needed to update the conditional probabilities halfway our study. For several CPS cases this resulted in different most informative manifestations. For many of these most informative manifestations we had to report 'unknown' because these manifestations were not asked during the initial interview. Thus the posterior probabilities after the most informative manifestations were based on iasufficient information. When all manifestations registered during the initial interview were reported as additional manifestations an increase of the posterior probabilities for CPS was observed. Therefore one should ignore the number of correct classifications after the informative manifestations in Table 1 for CPS. For the other seizure types this effect is not observed because the most informative manifestations remained largely the same although the sequence in which they were asked changed.

After each step in the protocoll we determined a threshold for the maximum posterior probability such that no wrong classifications are made. A posterior probability for a seizure type $>0.97$ after reporting key manifestations indicated a correct classification. When this threshold is not reached one should continue to elicit the most informative manifestations. After all informative manifestations are recorded a posterior probability for a seizure type $>0.91$ indicates again a correct classification. For the remaining cases additional manifestations should be reported. As soon as a posterior probability reaches 0.91 one can stop the elicitation process. When there are no manifestations to be reported, e.g. because the 
parient or relative cannot provide a more detailed description and the probability is less than 0.91, the classification may not be correct. Again we conclude that additional manifestations seem to add less to the accuracy of DSS, unless the posterior probability of 0.91 is not reached after the informative manifestations.

The key and informative manifestations provide highly relevant information to obrain the correct classification. These manifestations should always be asked to the patient or relative in classifying seizures.

From the analysis of the cases that were classified incorrectly by the system and also incorrectly classified by the epileptologist based on the reported manifestations it seems that the patient's description of the manifestations was incomplete for these cases or that other information has provided evidence for the treating neurologist to make another diagnosis.

\section{Limitations of SICS}

Only five seizure types were included in the system. These five selected seizure types are the most frequently occurring seizure types in adolescents and adults.

Eleven patients were suffering from secondary generalized TCS according to the diagnoses of the epileptologists. Because for primary or secondary generalized tonic-clonic seizures all anti-epileptic drugs (AEDs) are in general acceptable, except ethosuccimide that is only indicated for absence seizures, the primary and secondary generalized TCS were put together in one category. A distinction between these types of seizures cannot be made by SICS since it is not able to reason with temporal ordering of the manifestations.

The system can only be used when a physician is certain that the patient has epilepsy but still has to determine the kind of seizure type the patient is suffering from. The system is not built for the differential diagnosis of epileptic-like phenomena.

\section{Recommendations}

The accuracy of the system in this efficacy study is quite good. This is a strong indication that the DRFs of the included five seizure types are valid. The next step is to test the system in a clinical setting e.g. a general neurology department/clinic.

It is also possible to use the system in educational settings. Medical students and/or neurology residents can be trained to ask and report manifestations in a systematic way by using simulation patients. When these students or residents are later confronted with epilepsy patients in daily practice they may have developed a strategy to diagnose the seizure type.

\section{Gavistion}

This efficacy study of the DRFs showed an accuracy of $91 \%$ of SICS for the most common seizure types. This indicates that the knowledge encoded in the DRFs is valid. The next step is to test the DRFs in a clinical setting to evaluate the applicability in every daily practice. 


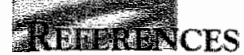

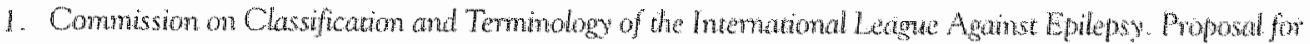

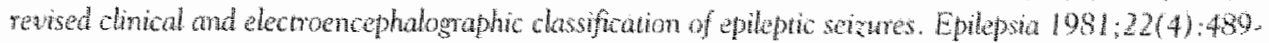
501.

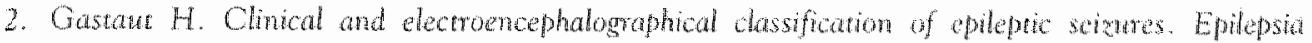
$1970 \cdot 11(1): 102-13$

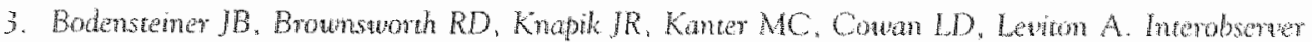
variabliry in the LAE classification of seimes in childhod. Epillessia 1988;29(2):123-8.

4. van Donselnar CA, Geens AT, Meustee J. Habbema JD, Stan A. Rethabiling of the diagnosis of a firt socatre. Neurology 1989;39(2 Pi 1):267-71.

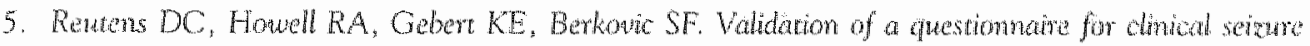
diagnosis. Epilepsia 1992;33(6):1065-71.

6. Sander JW, Shorwon SD. Incidence and prewatence sudies in epilepsy and the metholological problems: al review. J Newn Neurosurg Psychiatry 1987;50(7):829-39.

7. Engelbreche $R$, Recior A, Moser W. Verification and walidation. In: Van Gemip EMS, Tabmon II., editors. Assessment and eqahation of information sechnologies. Amstedam: 105 Press; $1995,51-66$.

8. vam Ast JF, Talmon JL, Renier WO, Hasman. A. An approach to knowledge base construction based on expert opinions. Methods Inf Med 2004:43:427,32.

9. Brender J, Tamon J, Nykanen P. McNair P, Demeester M, Betscast R. On the evaluation of Bstem integration. In: Van Genmip EMS, Tamon JL. Assesment and evaluaton of infomation redualoges. Amsterdan: 10S Press; 1995:189-208.

10. Rector AL, Ackerman E. Rules for seduentiad diamosis. Comput Biomed Res 1975,8:143155.

11. Gonry GA, Bamet GO. Sequential diagnosis by computer. JAMA 1968;205(12),849-54.

12. Nahr C. From assessment to decision-making. In: Van Gemip EMS, Talmon UL. Assessnenr and equination of infomation technologies. Amsterdam: IOS Pres; 1995:117-126.

13. Commission on Classificanon and Teminology of the International League Agranst Epilepsy. Proposal for rewised classification of epilepsies and epilaptic syndromes. Epilepsia 1989;30(4):389-99.

14. Korpinen L, Pietila T, Peltola J, Nissila M, Keranen T. Toumen T. Peranek ES, Frey H. Evalwation of Epilepsy Expert-a deciston suppont system. Comput Methods Programs Biomed 1994:45(3):223-31.

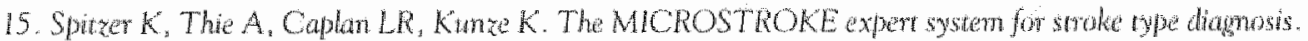
Srote $1989: 20(10): 1353-6$ 


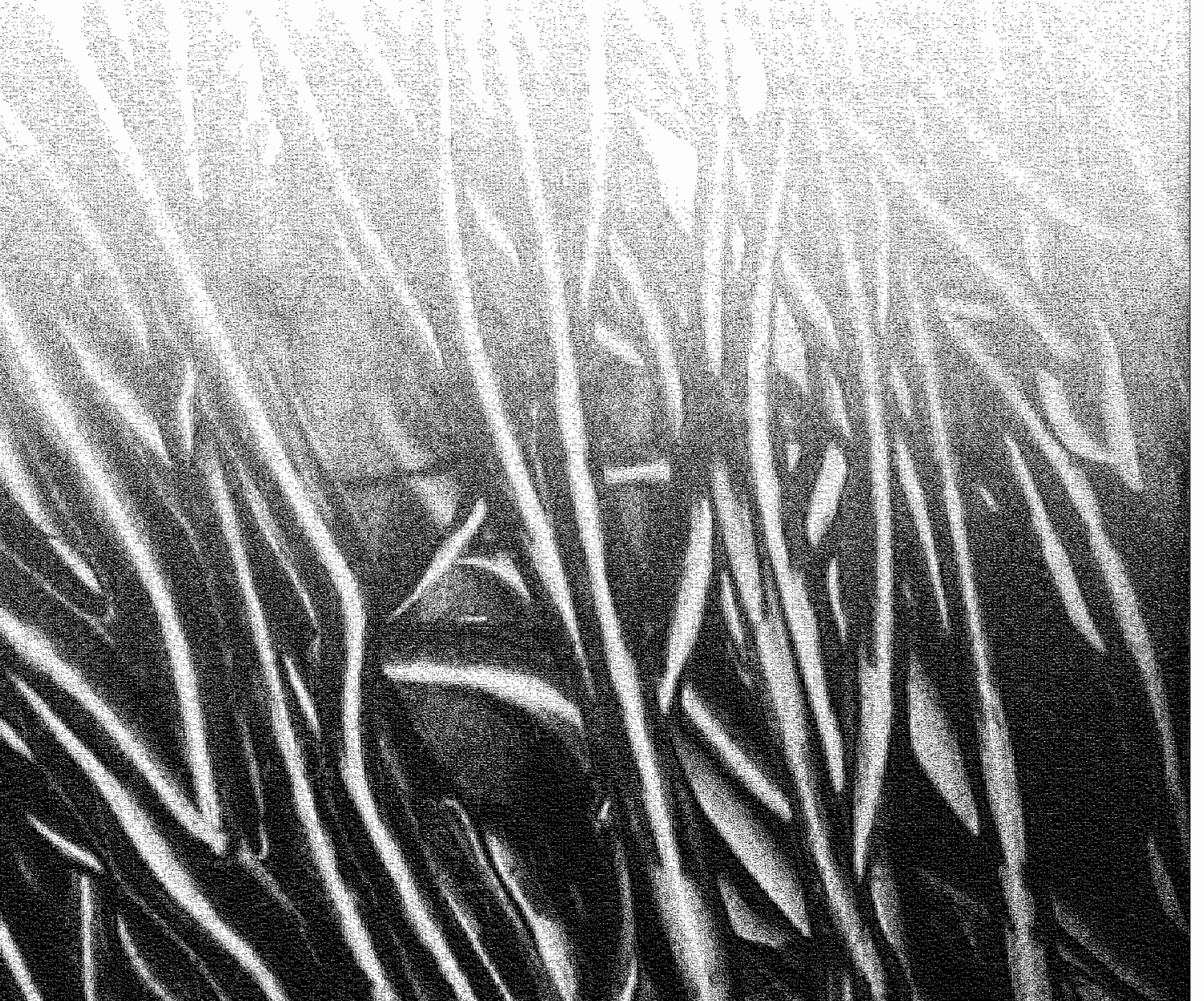




\section{Chapter 7}

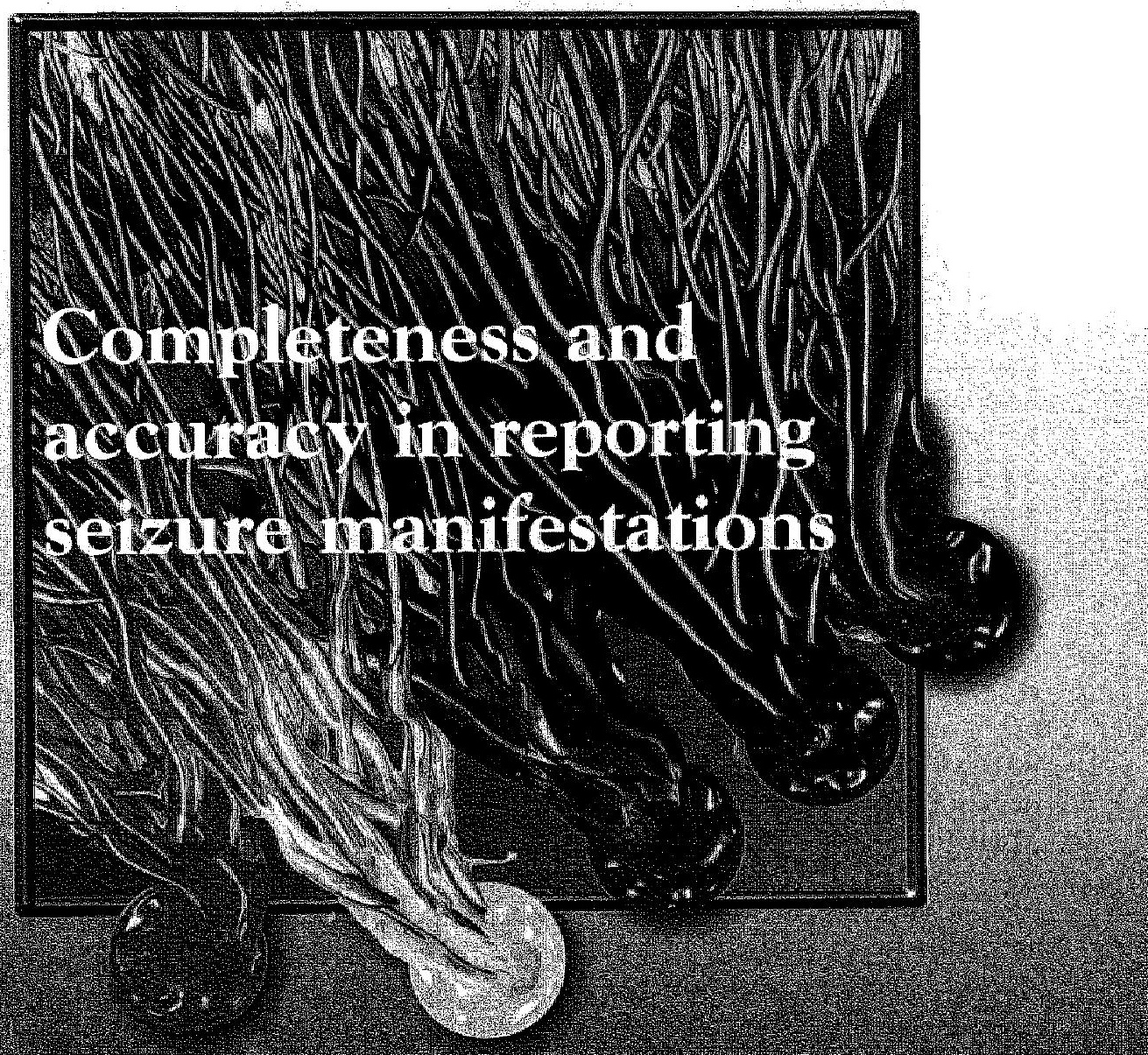




\section{楼}

Observing and reporting signs and symptoms is essential to diagnosis. A sign is defined as "any objective evidence of the presence of a disease or disorder' and a symptom as 'any sensation or change in bodily function experienced by a patient that is associated with a particular disease" [1]. Signs and symptoms are both disease manifestations.

An objective and complete description of someone's disease manifestations provides physicians the information about the patient's condition essential for patient management and scientific exchange.

Similarly, for the classfication of epileptic seizures a complete description of the seizure expression is necessary 121 . A complicating factor is that in most cases a physician does not witness the seizure. Furthermore seizures can be very short or subtle and therefore difficult to observe. In practice, the classification of epileptic seizures depends highly on the seizure manifestations as reported by the patient and/or his or her relatives. The completeness with which the seizure manifestations are described has implications for the classification of the seizure and the choice of treatment $[3]$, which is illustrated in the study of Bodensteiner et al. [2]. The objective of Bodensteiner"s study was to assess the inter-observer variability in classifying seizures according to the International Classification of Seizures (ICES). One senior neurologist and three neurology residents independently reviewed 2.219 verbatim descriprions of seizure manifestations and classified these descriptions using the seizure types included in the International Classification of Epileptic Seizures of 1981 [4]. Of these descriptions $22 \%$ to $51 \%$, depending on the level of training of the physicians, were considered unclassifiable due to insufficiendy described seizure manifestations. This indicates that complete descriptions of patient manifestations facilitate communication about the seizures of patients among neurologists.

Medical students and neurology residents are trained to ask relevant information and to observe and report seizure manifestations because only then will they be able to correctly classify seizure types. However, the training of students and residents is focused on observing and reporting abnormal seizure manifestations according to lists in textbooks. The fact that a particular abnormal manifestation is not present may play an important role in establishing the diagnosis and discriminating between seizure types. Therefore we will distingusth in the following abnomal and normal manifestations. Abnomal manifestations are defined as the signs and symptoms that occur when a patient is having a seizure, e.g. clonic movements of arms and legs. Normal manifestations are defined as that part of the behaviour of the patient that is not affected by the actual seizure but which may be affected in other seizure types, e.g. consciousness unchanged.

The accuracy of the diagnosis depends on the capability of students and residents to correctly identify relevant normal and abnormal manifesitations. In this observational study we focus on the quality of reporting seizure manifestations by medical students and neurology residents and the potential impact of incomplete and erroneous reporting on the quality of the diagnosis.

The purpose of the present study is a) to obtain insight in how completely medical students and neurology residents report relevant seizure manifestations b) to assess the potential impact of including normal manifestations on the classification of seizures and c) to make recommendations for training in observing and reporting seizure manifestations. In this paper the following research questions will be answered: 
a) How completely do medical students and neurology residents with different levels of experience report seizure manifestations?

b) Are medical students and neurology residents able to make the cortect seizure classification?

c) What is the potential impact of reported normal manifestations next to abnormal manifestations on the classification of the seizure types?

\section{trit}

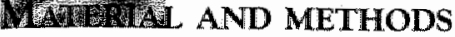

\section{Participants}

Neurology residents and medical students from two different training hospitals participated in this study. One group was from a university hospital and the other from a general hospital with training facilities. For each group a separate session was organized.

Ten neurology residents, two residents not in training and two medical students participated in this study. The two residents not in training carried out their PhD work on a topic related to neurology but not to epilepsy. The two medical students were in their clinical training period on the neurology ward.

We defined three groups of participants based on their degree of experience in epilepsy (see Table 1). One medical student did not report manifestations and did not classify the seizure for video 1 due to late arrival in the session.

\section{University hospital General hospital Tota}

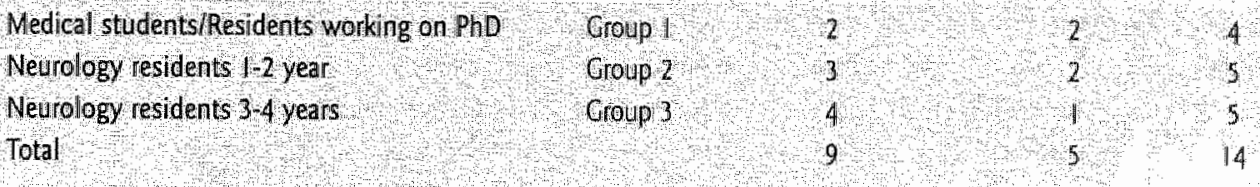

Table 1. Overview of participants

In both training hospitals once a year, all neurology residents together receive a theoretical training about epilepsy. Their experience increases in clinical practice when they are observing and treating patients on the ward and in the outpatient clinic.

\section{Method of data acquisition}

All participants were asked to report seizure manifestations of four cases presented via video recordings of the seizures. The epileptologist (WR) responsible for the training in epilepsy in one of the training hospitals selected the videos.

The four seizure types are briefly described in Table 2. Each of the seizure rypes showed seizure manifestations that are highly characteristic for the particular seizure type. The sound tracks on the videos were not used, because on the original recordings a reporter explained the seizure manifestations. However, sounds made by the patient as well as other relevant information on the sound track during the seizure were given by the epileptologists or the epilepsy researcher (W/VA).

The epileptologist made a referee report of the abnormal manifestations which served as the reference standard (golden standard). 
We developed a form with a structured list of 120 abnomal manifestations. The participants from the university hospital were asked to tick off the relevant observed abnormal manifestations.

\begin{tabular}{|c|c|c|c|c|}
\hline 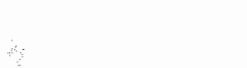 & Video I & Video 2 & Video 3 & Video 4 \\
\hline Selzure type & $\begin{array}{l}\text { Complex } \\
\text { partial sefzure }\end{array}$ & $\begin{array}{l}\text { Typical } \\
\text { absence seizure }\end{array}$ & $\begin{array}{l}\text { Simple } \\
\text { partial seizure }\end{array}$ & $\begin{array}{l}\text { Myoclonic } \\
\text { serzure }\end{array}$ \\
\hline Duration & 262 seconds & 15 seconds & 61 seconds & 29 seconds \\
\hline $\begin{array}{l}\text { Number of abnormal } \\
\text { manifestations } \\
\text { (selected by referee) }\end{array}$ & 24 manilestations & 10 manifestitations & 19 manifestations & 10 manifestations \\
\hline \multicolumn{5}{|l|}{ Key marifestations: } \\
\hline $\begin{array}{l}\text { "Onset } \\
\text { Movernents }\end{array}$ & $\begin{array}{l}\text { Rapidly gradual } \\
\text { Gestural automatisms: } \\
\text { bilateral; asymmetric }\end{array}$ & $\begin{array}{l}\text { Sudden } \\
\text { (Eyelid and lip) } \\
\text { myodonias: bilaterat }\end{array}$ & $\begin{array}{l}\text { Rapidly gradual } \\
\text { Leg clonic. } \\
\text { tonic unilateral }\end{array}$ & $\begin{array}{l}\text { Sudden } \\
\text { Leg myoclonias: } \\
\text { bilateral }\end{array}$ \\
\hline "Consciousness & $\begin{array}{l}\text { Stops normal ongoing } \\
\text { activity, unresponsive } \\
\text { to spaken words and } \\
\text { visual stimuit, rapidly } \\
\text { gradual loss of } \\
\text { consciousness }\end{array}$ & $\begin{array}{l}\text { Stops ongoing } \\
\text { activity, abrupt } \\
\text { sudden loss }\end{array}$ & Preserved & Preserved \\
\hline $\begin{array}{l}\text { Relevant nomal } \\
\text { manifestations }\end{array}$ & $\begin{array}{l}\text { No generalized tonic } \\
\text { or clonjic movements }\end{array}$ & $\begin{array}{l}\text { No post-ictal } \\
\text { phenomena }\end{array}$ & $\begin{array}{l}\text { No influence on } \\
\text { consciousness }\end{array}$ & $\begin{array}{l}\text { No influence on } \\
\text { consciousness }\end{array}$ \\
\hline 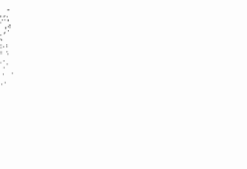 & No falling asteep & No lalling asleep & $\begin{array}{l}\text { No post-ictal } \\
\text { phenomena } \\
\text { No falling asleep } \\
\text { No automausms }\end{array}$ & No falling asleep \\
\hline
\end{tabular}

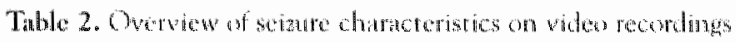

To be able to assess the porential role of normal manifestations in diagnosing we revised the form for the general hospital by including the option to report explicitly the absence of an ahnormal manifestarion when they consider that to be relevant for making the diagnosis.

Before starting the videos the parricipants had the opportunity to ask for a clarification of terms on the list. The explanation was given by the epileptologist or the epilepsy researcher. In both settings only a few terms neeted to be explained.

The videos were shown to the participants only once. Afrer each video they had the opportunity to report the observed manifestations and their clinical diagnosis (seizure type). This took ahout $3-5$ minutes per video. After all forms were collected the resuls were discussed by looking again to the videos togerher with the epileptologist or epilepsy researcher.

\section{Analyses}

To be able to relate the reported manifestations with a diagnosis we used the Support in Classification of Seizures (SICS), a decision support system. SICS can support medical 
students and neurology residents in the registration of patient manifestations and in the classification of seizures. SICS uses structured descriptions of the various seizure types consisting of (semi-) quantitative estimates of the frequency of occurrence of a large number of manifestations [5]. SICS can only be used when it is clear that the patient is suffering from epileptic seizures. The goal of SICS is to determine the seizure type based on reported seizure manifestations and Bayes' rule. The seizure rype with the highest posterior probability is considered as the diagnosis made by SICS. Preliminary results on 66 patients with different seizure rypes showed an agreement of $91 \%$ between the classification of SICS and the neurologists' diagnosis.

\section{Validity of the golden standard}

The validity of the golden standard was determined by entering the abnormal manifestations reported by the referee epileptologist in SICS and determining whether the seizure type with the highest posterior probability was equal to the seizure type shown on the video.

We also determined whether there are manifestations reported in the golden standard but not by any of the participants and manifestations reported by the participants but not in the golden standard.

\section{Completeness in recording and diagnostic accuracy}

To determine the correctness and completeness of the seizure manifestations reported by the participants we compared the abnormal manifestations reported by the participants with the abnomal manifestations reported in the golden standard. We determined the number of correct-positive and false positive abnomal manifestations reported.

To determine the diagnostic accuracy of the participants we compared the seizure classification provided by the participants with the seizure type of the video. To assess whether the abnormal manifestations reported by the participants are in accordance with the seizure type they diagnosed we entered the reported manifestations in SICS and compared the classification provided by SICS with the diagnosis of the participant.

The Kruskal-Wallis test was performed to assess whether the groups of participants significantly differed in the number of reported seizure manifestations for each wideo. The chi-square test was used to compare the diagnostic accuracy among the groups of participants.

\section{Impact of recording nomal manifestations}

The impact of reporting normal manifestations on the classification of seizures wass assessed by using the data of the participants from the general hospital. All abnormal manifestations reported by the participants were entered in SICS by the researcher resulting in posterior probabilities of the different seizure types (PI). Then all normal manifestations reported by the participants were added and the posterior probabilities are determined again (P2). The differences between the probabilities PI and P2 indicate the effect of explicitly reporting normal manifestations on the classification of seizures. We expect that the probability of the cortect seizure type will increase whon tiormal manifestations are reported. 


\section{Validity of the golden standard}

Entering the abnormal manifestations reported by the referee epileptologist in SICS resulted in the correct diagnosis of the seizure type for all four video cases.

Manifestations reported by the participants but not in the golden standard have been checked. None of them were visible on the videos. This is an indication that the golden standard is complete.

Manifestations in the golden standard that were not reported by any of the participants

Participante
Correct-positive manifestations
False-positive manilestations

Video I: \# of registered manifestations in golden standard $=24$

$\begin{array}{lcccccc} & \text { Mean } & \text { SD } & \text { Mean } & \text { SD } & \text { Mean } & \text { SD } \\ \text { Medical students }(n=3 *) & 15.25 & 10.5 & 7.75 & 5.3 & 7.5 & 5.5 \\ \text { Residents } 1-2 \text { yr }(n=5) & 16.2 & 4.6 & 9.2 & 3.3 & 7.0 & 1.6 \\ \text { Residents } 3-4 \text { yr }(n=5) & 19.0 & 1.9 & 10.6 & 1.8 & 8.4 & 3.4 \\ \text { Kruskal-Wallis test p-value } & 0.724 & & 0.661 & & 0.546\end{array}$

Video 2: \# of registered manifestations in golden standard $=10$

$\begin{array}{lcccccc} & \text { Mean } & \text { SD } & \text { Mean } & \text { SD } & \text { Mean } & \text { SD } \\ \text { Medical students }(n=4) & 11.25 & 2.8 & 5.25 & 0.5 & 6.0 & 2.4 \\ \text { Residents } 1-2 \text { yr }(n=5) & 11.0 & 2.2 & 4.6 & 1.7 & 6.4 & 2.6 \\ \text { Residents } 3-4 \text { yr }(n=5) & 11.6 & 2.2 & 6.0 & 1.2 & 5.6 & 1.7 \\ \text { Kruskal-Wallis test p-value } & 0.913 & & 0.384 & & 0.858 & \end{array}$

Video 3: \# of registered manifestations in goiden standard = 19

$\begin{array}{lcccccc} & \text { Mean } & \text { SD } & \text { Mean } & \text { SD } & \text { Mean } & \text { SD } \\ \text { Medical students }(n=4) & 17.0 & 2.4 & 10.25 & 0.5 & 6.75 & 2.6 \\ \text { Residents } 1-2 \text { yr }(n=5) & 17.2 & 5.0 & 8.8 & 2.4 & 8.4 & 3.4 \\ \text { Résidents } 3-4 \text { yr }(n=5) & 19.6 & 4.3 & 9.0 & 2.0 & 10.6 & 4.0 \\ \text { Kruskal-Wallis test p-value } & 0.585 & & 0.421 & & 0.306 & \end{array}$

Video 4: $\#$ of registered manifestations in golden standard $=10$

$\begin{array}{lcccccc} & \text { Mean } & \text { SD } & \text { Mean } & \text { SD } & \text { Mean } & \text { SD } \\ \text { Medical students }(n=4) & 11.0 & 1.6 & 7.0 & 1.4 & 4.0 & 2.4 \\ \text { Residents } 1-2 \text { yr }(n=5) & 10.4 & 0.6 & 7.0 & 1.6 & 3.4 & 2.1 \\ \text { Residents } 3-4 \text { yr }(n=5) & 10.0 & 1.7 & 6.8 & 0.8 & 3.2 & 1.3 \\ \text { Kruskal-Wallis test p-value } & 0.352 & & 0.988 & & 0.906\end{array}$

*One medical student did not register manifestations for video I.

Table 3. The number of correct-positive and false-positive abnomal manifestations reported by the participants fur axch villoo 
were also checked. Only a few of them were difficult to recognize on the video and mighe have been incorporated in the golden standard due to additional knowledge mentioned on the sound track of the video.

\section{Completeness in recording}

The manifestations reported by the participants were compared with the manifestations reported in the golden standard. Table 3 gives an overview of the mean and standard deviation of the reported correct-positive and false-positive abnomal manifestations. For video 1 the participants reported on average $38.3 \%$ correct positive manifestations, for video $252.0 \%$, for video $349.5 \%$ and for video $469.0 \%$. We identified no significant differences in the total number of reported manifestations berween the groups of participants, nor in the number of reported correct-positive and false-positive manifestations (Table 3). Therefore we pooled the data for the different groups of participants to compute means and standard deviations.

The majority of the participants reported manifestations related to 'onset of the seizure", 'cessation of the seizure', 'duration of the seizure', 'course of seizure', 'influence of seizure on consciousness' and 'speed of loss of consciousness' in all cases. These manifestations reported by the participants were not always in accordance with the manifestations reported in the golden standard. E.g. the duration of the typical absence seizure (video 2) was 15 seconds. The referee epileptologist selected a duration of 10-30 seconds while 12 of the participants selected a duration of $<10$ seconds.

\section{Diagnostic accuracy}

The seizure classifications given by the participants were compared with the seizure types shown on the videos (Table 4; column 1). The results show no significant differences between the three groups of participants $(\chi 2=4.10 ; p=0.128)$.

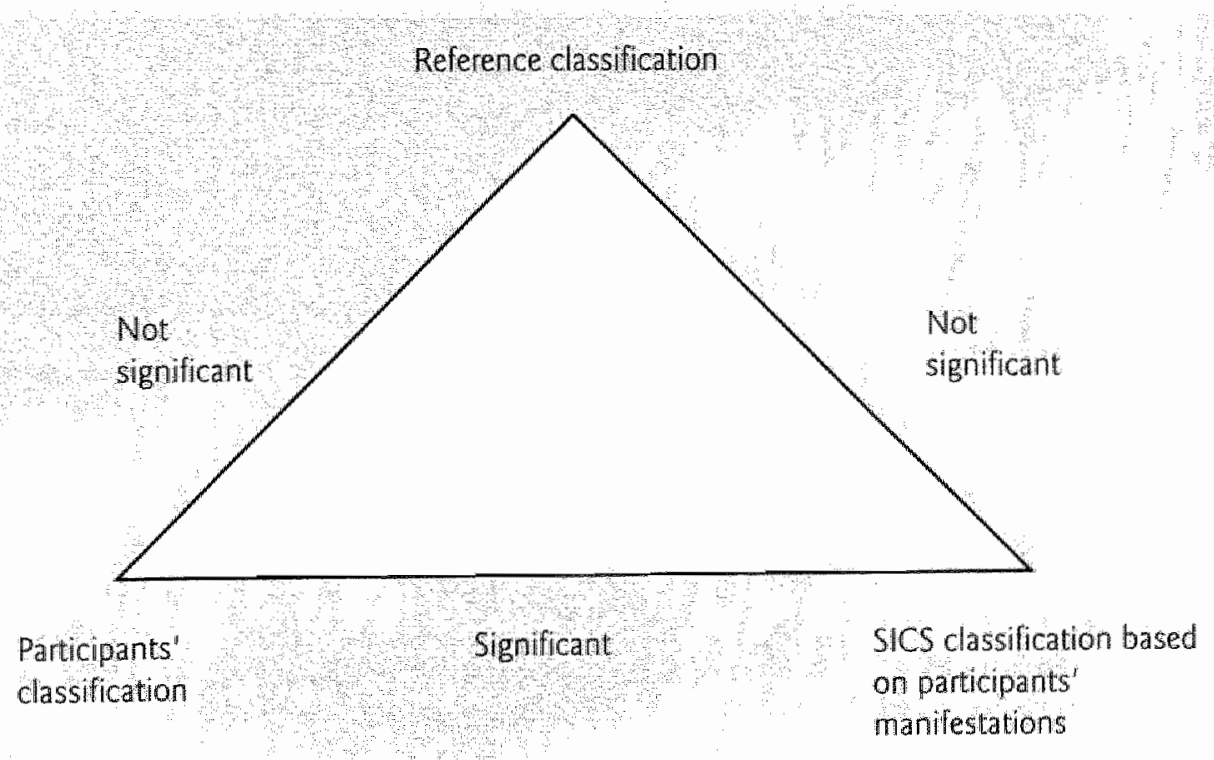

Figure 1. Diagnostic accuracy summarized 
We investigated in how many cases the seizure classification of the participants is in accordance with the seizure classification made by SICS based on the abnormal manifestations reported by the participant (Table $4 ;$ column 2 ). These results show significant differences between the three groups of participants $\chi_{2}^{2}=7.86 ; p=0.020$ ).

Finally no significant differences between the groups of participants were found in how often SICS's classification based on the reported manifestations is in accordance with the seizure types shown on the videos (Table 4; column $3 ; \chi 2=0.518 ; p=0.772$ ).

Figure 1 summarizes the assessment of the diagnostic accuracy.

\section{Impact of reporting normal manifestations}

The results of the potential impact of reporting normal manifestations on the seizure classification are presented for the participants from the general hospital. Only two of the five participants registered such manifestations for all four seizure types. The number of reported normal manifestations varied between one to ten (for one case 23).

Figure 2 presents how the posterior probabilities based on all reported abnormal manifestations $(P 1)$ change when normal manifestations are added (P2). When normal

$$
\begin{array}{ccc}
\begin{array}{c}
\text { \# of part. classifying } \\
\text { the correct seizure }
\end{array} & \text { Total \# of times part. } & \text { \# of times SICS } \\
\text { type (column 1) } & \text { classifification (column 2) } & \text { seizure type (column 3) }
\end{array}
$$

Medical students (4)

Video ${ }^{*}$

Videa 2

Video 3

Video 4

Total

1
2
0
1
$4(15)$

$\begin{array}{cc}0 & 1 \\ 0 & 1 \\ 1 & 1 \\ 1 & 2 \\ 2(15) & 5(15)\end{array}$

Residents $1-2$ year $(5)^{* *}$

Video I

Video 2

Video 3

Video 4

Total

3
3
2
2
$10(16)$

3
0
1
2
$6(16)$

3

0

0

3

Residents $3-4$ year ( 5 )

Video I

Video 2

Video 3

Video 4

Total

4
1
1
4
$10(20)$

3
1
5
3

* One medical student did not report manifestations and did not provide a seizure classilication.

* One resiclent in histher $1-2$ year did not provide a seizure classification, but reported manifestations.

Table 4. Diagnostic accuracy for abonomal manifestations 
manifestations are entered in SICS the number of correct diagnoses increases (SICS's classification is in accordance with the seizure type on the video): for video 2 all cases became correctly diagnosed, for video 3 one of the three cases became correct and for video 4 both cases became correctly diagnosed.

For three of the four participants (Part 1, Part 12 and Part 14) the posterior probabilities of the correct seizure type of video 1. decreased slightly when normal manifestations were added. Cases for which a participant did not report normal manifestations were not included in Figure 2.

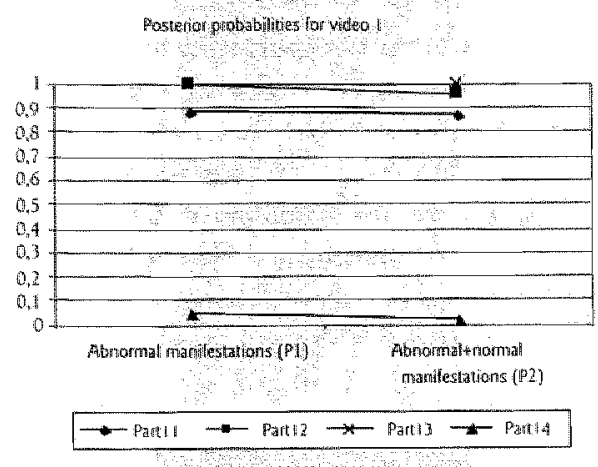

Posterion pooblbilities for sideos

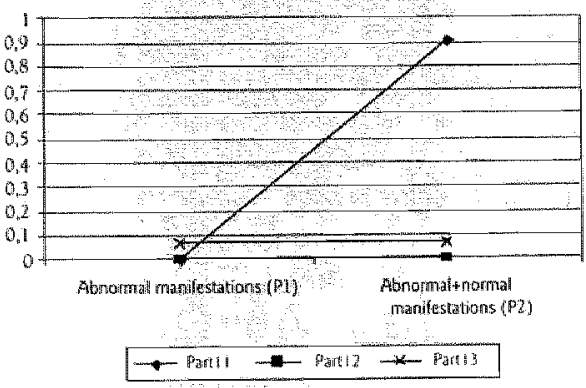

Fosterior pobothities lor widino?

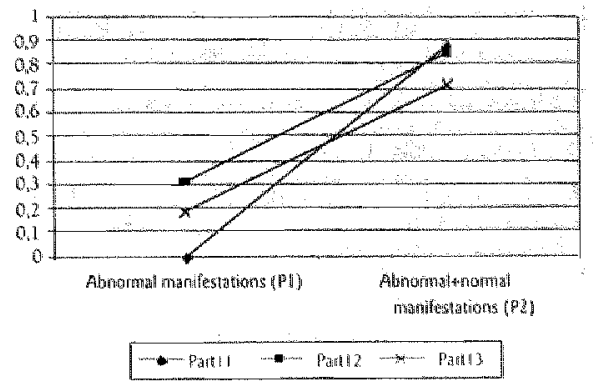

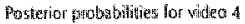

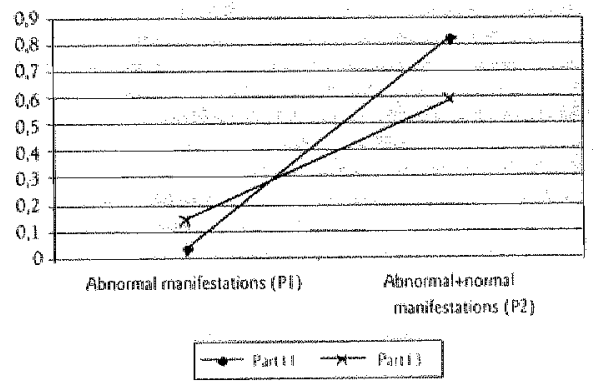

Figure 2. Difference in posterior probabilites based on the reported abmormal and normal manthestivans for the conect seivure twpe for participants from the genaral hospital

\section{Thetession}

\section{The main research findings}

Medical students and neurology residents observing patients with an epileptic seizure on a video provide an incomplete description of the seizure manifestations and also report manifestations not present on the videos. This phenomenon was observed for each of the videos. The participants also have difficulties in diagnosing the correct seizure type.

When SICS is used to classify seizure types we observed that entering normal manifestations next to abnormal manifestations resulted in more correct seizure classifications and in an increase in the posterior probabilities for already correctly classified seizures. 


\section{Completeness in recording}

Neurology residents in their third or fourth year do not significantly report seizure manifestations more completely than medical students or residents in their first or second year. It was expected that the residents with more experience would be more complete and accurate in reporting manifestations but this hypothesis is rejected. This can be explained in several ways.

First the participants are trained and used to ask the patient or his/her relative(s) to describe the seizure manifestations. In this study participants were asked to report manifestations they observed. This requires additional skills.

Secondly, we found that in almost all cases the participants reported manifestations related to 'onset of the seizure", "cessation of the seizure', 'duration of the seizure' and 'course of seizure', which are described by mutually exclusive manifestations that can be ranked. For example 'onset of seizure" can be described as "slowly gradual - rapidly gradual - sudden - difficult to identify". Although full agreement between the participants and the golden standard is preferred one category off for these manifestations may be considered as an acceptable response because in a clinical sense the difference between the manifestations is small and maybe difficult to observe. This increased the number of correct-positive manifestations reported by the participants (Table 5).

\begin{tabular}{|c|c|c|c|c|c|c|c|c|}
\hline & Video 1 & & Yideo? & & Video 3 & & Video 4 & \\
\hline & Bétore & Alter & Before & After: & Before & After & Before & After \\
\hline Onsel of seizure & 3 & 12 & 12 & 14 & 0 & 10 & 13 & 3 \\
\hline Cessation of seizure & 1 & 1 & 10 & 12 & 8 & 12 & 12 & 12 \\
\hline Duration of seizure & 8 & 12 & 2 & 14 & 6 & 14 & 13 & 14 \\
\hline Course of seizure & 5 & 8 & 10 & 10 & 9 & 10 & 9 & 9 \\
\hline
\end{tabular}

Table 5. Number of correct-positive mutually exclusive manifestations before correction and after correction for one category off

When the epileptologist or epilepsy researcher described sounds made by the patient and provided other relevant information present on the sound track it may be expected that all participants were triggered to record it. We found that not all participants recorded this verbal information. E.g. when showing video 1 it was mentioned that the patient was screaming at the onset of the seizure. Since only 11 of the 13 participants reported this manifestation apparently not all participants considered this item relevant. This confirms the assumption that participants only reported manifestations he/she considers to be relevant for the diagnosis.

Finally, participants may have observed a manifestation, but only at an unconscious level. They may even have taken this manifestation into account for diagnosis, but they did not consciously report it.

\section{Diagnostic accuracy}

By determining in how many cases the participant's classification was in accordance wirh the classification given by SICS based on the reported abnormal manifestations we could 
infer whether the participants were able to translate their observed and reported manifestations into the corresponding seizure classification. In only 20 of the 51 cases the participant's classification is the same as SICS's classification. This indicates that medical students and residents are not fully able to link observed manifestations with an appropriate seizure type. It must be noted that the participants significantly scored better with increasing experience.

In 20 of the 51 cases SICS classification based on the manifestations reported by the participants is the same as the seizure shown on the video, while the participants correctly classify the seizures in 24 of the 51 cases. This finding supports the hypothesis that the participants have observed some of the manifestations in an unconscious way and properly interpreted them in the diagnostic process although they did not explicitly report these manifestations.

Providing a wrong seizure classification can have implications for the choice of the therapy, but sometimes therapy is the same for two seizure types. E.g. four of the five residents in their third or fourth year of training diagnosed video 2 as 'atypical absence seizure', while the patient was suffering from a 'typical absence seizure'. Strictly speaking the diagnosis is wrong but this does not influence the therapy.

The participants in the university and general hospital were asked to report abnormal manifestations. The participants in the general hospital were also asked to report relevant normal manifestations. A difference in this questioning might have caused a bias in the diagnostic accuracy of the participants among the two settings. Using the chi-square test, however, we found no significant difference $(\chi 2=3.187 ; \mathrm{p}=0.203)$.

\section{Potential impact of reporting normal manifestations}

Participants from the general hospital were asked to report abnormal manifestations and normal manifestations relevant for determining the diagnosis. Because only two of the five participants (one medical student and one resident in his first or second year) reported such manifestations for all four seizure types we assume that the participants have difficulties in identifying potentially discriminating normal manifestations.

However, for the majority of the cases the results show that when normal manifestations are taken into account by SICS in addition to the abnormal manifestations the probabilities for the correct seizure type increased and the number of correct diagnoses increased. Gorry et al. found similar results and reported that if a normal finding of a laboratory test occurs this finding can be of value in differentiating among diagnoses [6].

\section{Recommendations for education}

We found a rather poor diagnostic accuracy for all participating groups. We also observed that medical students and neurology residents have difficulties in completely and accurately reporting seizure manifestations.

In this study the participants were asked to observe seizure attacks on videos, to record the observed manifestations and provide a diagnosis. We already mentioned that in practice the classification of epileptic seizures depends highly on the seizure manifestations as reported by the patient and/or his or her relatives. Observing and reporting seizures requires additional skills. Training medical students and neurology residents in observing seizure attacks either on videos or in the clinic is likely to enhance their ability to interpret the seizure descriptions of patients. 
SICS used in this study can support such a training. It guides the user in systematically reporting patient manifestations and may facilitate diagnosis and classification.

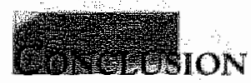

The difficulties in reporting relevant seizure manifestations and the poor diagnostic accuracy of medical students and neurology residents indicate that they need more clinical training in observing and reporting seizure attacks. A decision support system can be of value to support medical students and neurology residents in obtaining a complete description of the manifestations both affected and not affected by the seizure and support them in classifying the seizure type. 
1. Collins English Dictionary. Thid Edion. Harter Collins Publishers: 1992.

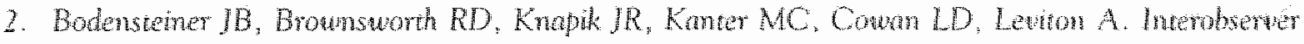
warialiliry in the ILAE classificanon of seivures in chidhood. Eptopsia 1988,29(2):123-8.

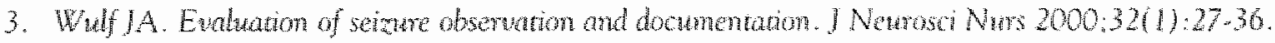

4. Commission on Classification and Teminologe of the ULA. Proposal for rasised clinical and elecroencephalographic classification of epteptic seivares. Eptlepsia 1981:2214):489-501.

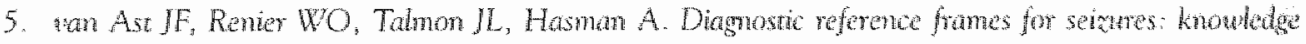
elicitarion and resules. 2004: Stbmitted.

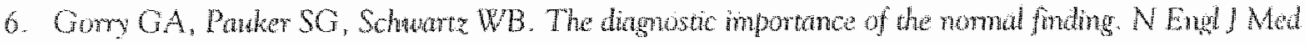
$1978 ; 298(9): 486-9$ 



\section{Chapter 8}

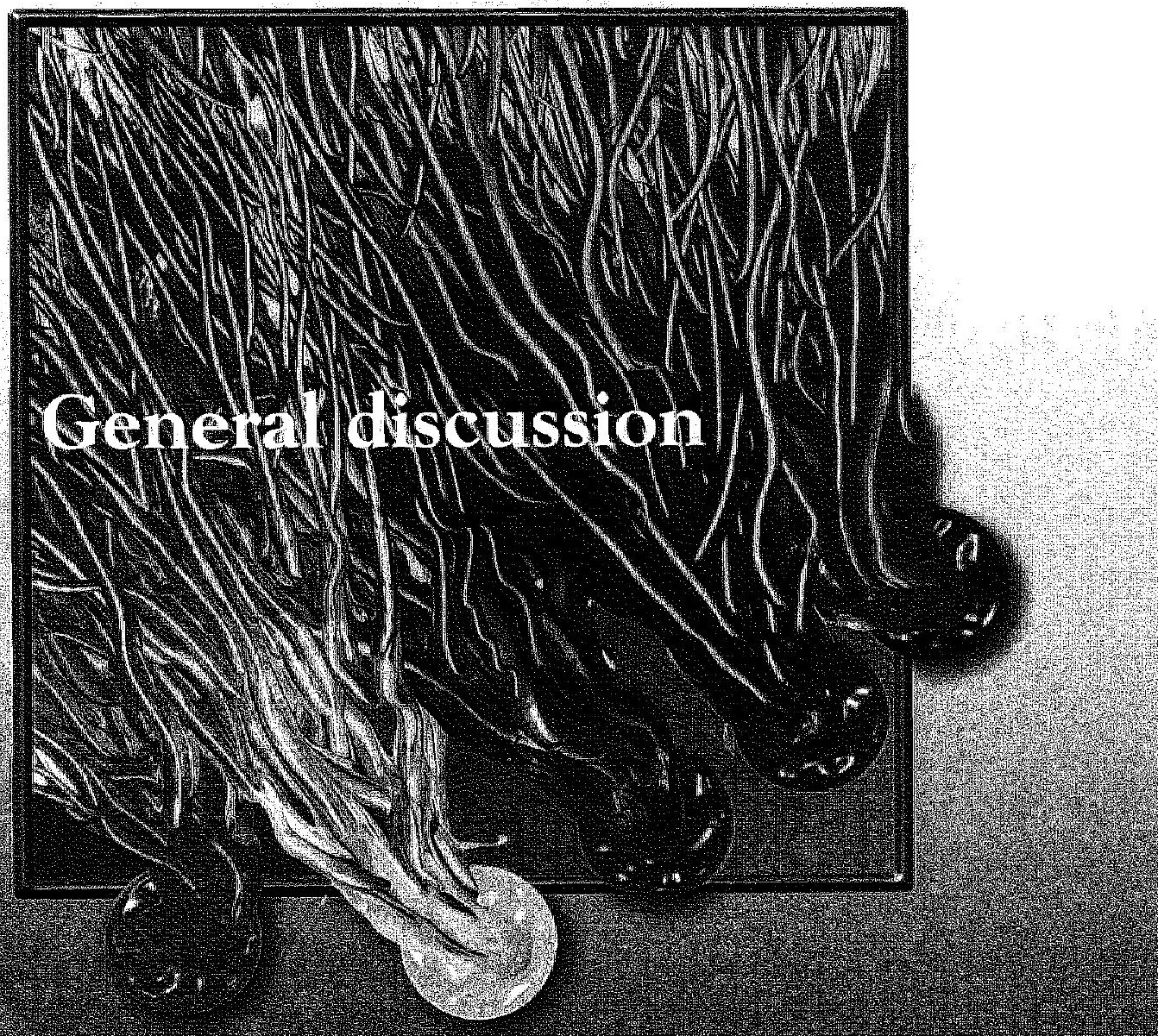




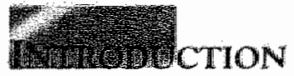

This thesis documents the development and evaluation of Diagnostic Reference Frames (DFRs) for epileptic seizures. A DRF is a structured description of the characteristics of a specific disease, e.g. of epileptic seizures. In this study the DRFs for seizures are based on knowlectge and expertise of experts in neurology and epileptology. The research described in this thesis focuses on the development of a methodology to elicit knowledge from experts, on the knowledge elicitation procedure irself and on the validation of the knowledge included in the DRFs.

In this chapter the main findings are summarized and the results are discussed. Further we discuss methodological aspects of the study and give recommendations for future research.

\section{W. HIDINGS}

The main conclusion of this thesis is that it is possible to develop reliable and valid DRFs for frequently occurring seizure types (simple partial seizures, complex partial seizures, myoclonic seizures, tonic-clonic seizures and typical absence seizureś) based on expert opinions.

We started with a pilot study to determine the feasibility of eliciting descriptive information on various seizure types from experts and to optimise the method for knowledge elicitation. In this pilot study we found that a group of neurologists and epileptologists reached a level of agreement concerning frequently occurring manifestations (manifestation present in at least $75 \%$ of the cases) that exceeded the expected agreement based on a pure random selection of manifestations. The experts selected a core of common, frequently occurring manifestations on which there was disagreement only on the core's extent. Each core of manifestations for a specific seizure type appeared to be different from the cores of the other seizure types. Based on the results of the pilot study we concluded that expert opinions can be used for building DRFs of seizure types.

The pilot study also helped to further shape the design of our main study. In the pilot study participants were asked to identify manifestations they considered present in at least 75\% of the cases of a particular seizure type. Apart from the fact that it is difficult to assess whether a manifestation occurs in more than $75 \%$ of the cases, rarely occurring manifestations that are highly specific for a particular seizure type are not obtained in this way. These manifestations are meaningful for the construction of DRFs because they have a high discriminative value. Therefore in the final study neurologists/epileptologists were asked to estimate the frequency of occurrence of the manifestations on a 5 -point scale.

In the pilot study the manifestations were ordered alphabetically. In the final study the manifestations are clustered in 8 main categories. This approach enhanced the overview on the various symptoms and focussed the attention of the participants to specific aspects of the behaviour of the patient that might be affected by the seizure.

In the final knowledge elicitation procedure we used a Delphi (-like) procedure as to further reduce observer variability. Neurologists/epileptologists were asked to estimate the frequency of occurrence of manifestations for the most frequently occurring seizure types. In the feedback round of the Delphi procedure they were asked to review the manifestations for which they provided an estimate that deviated from the mean estimate of the group of experts. 
The so elicited estimates of all experts were averaged for each manifestation in each seizure type to obtain the mean frequencies of occurrence tepresenting how often a manifestation occurs in a specific seizure type. By computing the inter-rater intraclass correlation coefficient (ICC) we were able to proof that the averaged frequency estimates derived from the estimates of the experts were reliable. The averaged frequency estimates were included in the DRFs. DRFs were developed for the most frequently occurring seizure types: simple partial seizures, complex partial seizures, myoclonic seizures, tonic-clonic seizures and typical absence seizures.

Data can be reliable, but not valid. The validity of the DRFs was determined by classifying 66 patient cases using a decision support system, called Support in the Classification of Seizures (SICS). SICS used a Bayesian approach that uses the conditional probabilities for five different seizure types as determined from the experts. SICS classified $91 \%$ of the 66 patient cases correctly.

A prerequisite for a good classification is that manifestations are correctly observed and reported. In an observational study we focussed on the quality of reporting seizure manifestations. We assessed the completeness and accutacy in reporting seizure manifestations shown on four video cases by medical students and neurology residents. Results show that the mean percentage of correct-positive manifestations reported by the participants ranged from $38.3 \%$ to $69.0 \%$. We found that this group of participants had difficulties in reporting relevant seizure manifestations. When the reported manifestations were registered in SICS for classification, SICS classified the same seizure as shown on the video in 20 of the 51 cases, while the participants correctly classify the seizures in 24 of the 51 cases. This indicates that participants have observed some manifestations at an unconscious level and properly interpreted them in the diagnostic process although they did not explicitly report these manifestations. The difficulties in reporting relevant seizure manifestations of medical students and neurology residents indicate that there is a need for more clinical training in observing and reporting seizure attacks. SICS can be of value to support medical students and neurology residents in obtaining a complete description of the manifestations both affected and not affected by the seizure and support them in classifying the seizure type.

In this section the strengths and weaknesses of the different stages in the development of the DRFs are discussed.

\section{Methodology for knowledge elicitation}

For the development of the DRFs we needed information about the frequency of occurrence of manifestations in several seizure types. There were several options to obtain this information: a review of the literature, the use of a database existing of data previously or prospectively collected from real-life patients or asking experts to give their estimates about the frequency of occurrence.

A limited review of the literature showed that the frequency of occurrence of manifestations is sometimes indicated by qualitative terms and sometimes quantitative information is given. In studies in which qualitative terms are used the authors used their own set of concepts to describe the frequencies of occurrence. In quantitative studies not only different study populations were included on which the frequencies of occurrence were 
based but the manifestations studied varied considerably and didn't cover the domain as completely as needed for our study. This made it impossible to perform a meta-analysis to obtain reliable and valid information on the frequencies of occurrence.

Leaper et al. reported about the difference in accuracy of a compurer-assisted diagnosis using data previously collected from real-life patients and using estimates of probabilities provided by a group of experts in 472 patient cases. When the computer system used the estimates from the data base the overall diagnostic accuracy was $91.1 \%$. When the clinicians' estimates were used a diagnostic accuracy of $82.2 \%$ was found [1]. They also found, however, that the system based on expert opinions was as good as the diagnostic performance of the experts themselves (accuracy of 79.9\%). They suggest that each computer assisted diagnostic system should use data from a large-scale, real-life survey. Such large-scale surveys require proper definitions of the diseases included and independent evidence for the classification (golden standard). These prerequisites are not available in the field of epilepsy and therefore no surveys are available. E.g. Leaper et al. used pathology reports as golden standard for the diagnosis of abdominal pain. Unfortunately in epilepsy there is no golden standard that determines which type of seizure the patient has. Moreover, seizures not always manifest the same signs and symptoms or the complete expression of a seizure type.

Hence we had to elicit estimates of probabilities from experts. We developed a general approach to assure that reliable averaged frequency estimates for each manifestation for various seizure types could be obtained. We used the inter-rater intraclass correlation coefficient (ICC) to assess the reliability. The ICC is the fraction of the total variance of the set of item values that is due to real differences between items and not to random or systematic estimation errors of the experts. When an ICC of 0.9 is reached only $10 \%$ of the variance is due to random or systematic errors of the experts and when that is the case it is generally assumed that the averaged frequencies derived from these estimates are reliable $[2,3]$.

The ICC plays a pivotal role in our knowledge acquisition methodology. It is not only used to assess the reliability of the set of responses given by the participants. We also used the ICC to determine the number of experts needed to obtain a reliable set of responses and to determine whether one or more experts differed significantly in opinion from the other experts.

For each seizure rype we assessed the consistency of the frequency estimates of the experts and found an $1 C C$ of 0.9 for the frequenty occurring seizure types except for atypical absence seizures. Based on the consistent frequency estimates it was possible to obtain reliable averaged frequency estimates for each manifestation. For atypical absence seizures we needed more participants to estimate the frequency of occurrence of manifestations because of the lack of typical characteristics of this seizure type.

To determine how many participants are needed to obtain an $I C C$ value of 0.9 we used the Spearman-Brown prophecy formula [3]. The results from this formula are only valid when the systematic and random errors of the experts follow the same distribution. In our study we first included a group of Dutch participants and based on their responses we calculated the ICC and the number of additional experts needed to obtain an ICC of 0.9 . We recruited the additional participants on an epilepsy congress.

To assess whether the two groups of participants could be considered as one population we introduced an approach (based on the 1CC) to identify experts with diverging opinions. We could show that the additional participants and the Dutch participants appear to be 
samples of the same population although we identified one neurologist among the Dutch participants with diverging opinions. Changes in the responses of the participant due to the feedback can result in the identification of another expert with diverging opinions. There are two strategies to assess whether the opinions of additional expers deviate from the group opinion. Firstly, the opinions of the group of experts that already responded (initially only a few) can be used as a reference and each time an additional expert is added, his/her opinions are evaluated against the reference group. When an expert provides consistent opinions his/her opinions are added to the reference opinion. A second stratregy is to compare the opinion of each participant with the opinions of all other participants after all participant's opinions are obtained. This latter strategy avoids locking in on opinions of already included participants, which in our opinion is a more valid way of identifying participants with diverging opinions. We followed the last strategy for identifying deviating opinions.

\section{Knowledge elicitation procedure}

We used a Delphi-like procedure to obtain as reliable as possible DRFs and not the nominal group technique. In a nominal group technique information from experts is gathered during a meeting. A disadvantage of the nominal group technique is that the decision-making can be dominated by one individual, which is not possible in a Delphi process. In a Delphi process an expert panel is asked to complete questionnaires focussing on a specific topic of interest in several rounds. An additional advantage of the Delphi process is that it is relatively easy to elicir the opinions of a group of experts by email and/or internet. Secondly, participants' anonymity is kept which probably encourages a greater frankness of opinion. Disadvantages are the drop-out of panel members in later rounds and the time it takes the researcher to prepare everything $[4,5]$.

Traditionally Delphi-procedures are paper-based. Nowadays we have email and internet facilities that ease the Delphi-technique. Snyder-Halpern et al. compared mailed versus emailed applications of the Delphi procedure. The email approach offered advantages in terms of time and costs but the response rate was lower than for the mailed approach [6]. Aniruddha et al. developed and implemented a metadata-driven Delphi rating on the interner which generated individualized reports for each participant describing the median rating and the disagreement index. Advantages of a web-based Delphi procedure are the elimination of errors inherent in manual data entry and the need for data entry validation [7]. We developed an electronic form on which the participants could fill out the ir estimates to avoid errors in manual data entry. For the Dutch participants the feedback was paper. based. For the congress-participants the feedback on the ten most diverging estimates was generated by the systerm. In both ways $26 \%$ of the feedback forms were not returned.

In our study we did not continue with the Delphi process until the experts ultimately reached full consensus. We carried out only one round of the Delphi process (response of the experts - analysis by the researcher - feedback to experts - response on feedback by experts - analysis). In addition we used the ICC to determine whether the frequency estimates were reliable enough. This Delphi-like procedure was carried out with the group of Dutch participants as well as with the group of congress participants.

The $1 \mathrm{CC}$ is not part of the standard Delphi process. In our Delphi-like procedure the $1 C C$ was used as a quality indicator of the gathered data. The $1 C C$ was determined during two stages in the procedure (before and after feedback).

We found that the feedback improved the reliability of the frequency estimates 
indicating that the Delphi (-like) procedure was successful in our study. In other medical domains, e.g. cardiology, the Delphi procedure also was applied successfully. Kors et al. needed a reference classification of electrocardiograms (ECGs) on which a group of cardiologists agreed upon. In a Delphi procedure of two rounds the agreement (kappa values measured) on the ECG-classification increased from 0.68 in round one to 0.81 in round two using six diagnostic categories [8]. In another study five cardiologists were asked to measure the onsets and offsets of warious ECG waves. A (modified) Delphi process of four rounds eliminated individual referee outliers (defined as a point estimate which differs considerably from the median referee results) resulting in precise and reproducible estimates serving as a standard for computer ECG measurement [9].

We recommend Delphi-like procedures for eliciting expert opinions. Including a measure of reliability of the results like the $\triangle C C$ adds credibility to the results.

\section{Validation of the DRFs}

The experts estimated the frequency of occurrence of the manifestations on a 5oint scale. We provided a frequency range for each of the five categories. The mid-points of these ranges were considered as the frequency estimates of the participants in the calculation of the average frequency to be included in the DRFs. We chose this approach because it is known that humans are poor frequency estimators [10]. It is much easier to select a frequency range. It is also to be expected that the participants would have provided "nice" frequencies $-1 \%, 5 \%, 10 \%, 25 \%$ etc - rather than accurate estimates.

For the most frequently occurring seizure types in adolescents and adults DRFs were developed in our study. These DRFs (manifestations with conditional probabilities for each included seizure type) were included in the decision support system called Support in Classification of Seizures (SICS). In our validation study patient manifestations were entered in SICS and by successively applying Bayes's rule posterior probabilities were calculated. The DRF with the highest posterior probability gives an indication of the classification of the seizure. We applied so-called 'idiot' Bayes i.e. we assume that variables are conditionally independent. In our study not all manifestations are independent. For practical reasons it was necessary to choose 'idiot' Bayes because it would be impossible to ask participants to provide all possible joint probabilities needed to take conditional dependence into account. Also building a Bayesian network that would properly model all dependencies is a task that requires a good understanding of the underlying processes that yield the various seizures and their manifestations.

Despite the fact that our DRFs are based on experts' opinions, rather than on frequency estimates derived from a (large) database $91 \%$ of the patient cases were correctly classified by SICS after the registration of the patient manifestations. When the accuracy of SICS is compared with the accuracy of two other decision support systems in neurology, SICS performed quite well: in Epilepsy Expert (a decision support system based on the International Classification of Epilepsies and Epileptic Syndromes able to discriminate between 36 categories) $72 \%$ of the 25 cases were correctly classified based on clinical findings [11]. In MICROSTROKE (an expert system for classifying 5 different stroke types) $72.8 \%$ of the 250 cases were correctly classified [12]. Whether an accuracy of $91 \%$ is sufficient for clinical practice is not yet proven. One needs to take into account the therapeutic, psychological and social consequences of a wrong classification. 
We followed a strict protocol for eliciting the manifestations from the patients. First some key manifestations were recorded. Next the advisory function of the system was used ten times to elicit the presence or absence of manifestations that could potentially cause the largest change in posterior probabilities. Finally, patients and their relatives were asked to mention manifestations that occurred during their seizures and that weren't addressed so far. Based on the behaviour of the posterior probabilities obtained in our set of 66 cases we defined a stopping strategy in such a way that no classification errors would occur when the protocoll would have been stopped before completion. Such a stopping rule could speed up the diagnostic process at the cost of a complete description of the seizures. Still the stopping rule should be walidated in a larger validation study.

SICS is not able to reason with a temporal ordering of manifestations. When a seizure is evolving from a partial to a generalized tonic-clonic seizure, the seizure is classified as 'tonicclonic' by SICS. For primary or secondary generalized tonic-clonic seizures all anti-epileptic drugs are in general acceptable except ethosuccimide that is only indicated for absence seizures. However, for providing the patient information on the disease and its consequences the physician must be aware of the fact that the impact of the seizures on daily life differs. When SICS is used for decision support in the classification of seizures, the physicians are responsible for identifying a possible temporal ordering of manifestations to provide correct information to the patient. It is conceivable that adding a question, whether the loss of consciousness coincides with the onset of the seizure in the case a TCS is identified while also manifestations are listed that could indicate a (complex) partial seizure, could eliminate this problem.

The inclusion of EEG-characteristics in the DRFs is questionable for several reasons. In our pilot study we concluded that the description of the EEG was too detailed and should be replaced by more general terms. In the final study we used more general terms but it still remains difficult to describe the EEG-characteristics in a sufficient and meaningful way. We found also very few EEG-characteristics that discriminate between seizure types. Furthermore early in the process of diagnosing seizures EEG-data are often not available and an EEG is a diagnostic tool and not a prerequisite for classification. Hence it seems advisable not to use this part of the DRF in clinical applications.

Since it is possible to obrain valid DRFs for the frequently occurring seizure types one can assume that a similar knowledge elicitation procedure will result in valid DRFs for other seizure types as well. It is difficult to say how many participants are needed to obtain reliable frequency estimates for the manifestations in these seizure types. The seizure types occur less frequently and therefore it is possible that the experts are less familiar with the frequencies of occurrence of the manifestations. When this is the case more neurologists may be needed than for obtaining reliable DRFs for the seizure types included in our study. On the other hand these seizure types may have a clear symptomatology which again will reduce the number of participants needed.

\section{WESOMANDATIONS AND IMPLICATIONS}

Because the efficacy study showed an accuracy of $91 \%$ of SICS for the most common seizure types, a validation in clinical practice is reasonable. Since we concluded that medical students and neurology residents have difficulties in reporting relevant seizure manifestations and have a poor diagnostic accuracy, we suggest that SICS might be offered to medical 
students and neurology residents during training and their residency respectively. In this way SICS can be validated in clitical practice and the residents can be trained in obserwing and reporting seizure attacks using the protocol for systematic registration of manifestations.

Five neurologists/epileptologists who participated in the knowledge elicitation procedure completed a small questionmaire about the applicability of decision support systems in epilepsy. They indicate that SICS might be useful as an educational tool for medical and nurse practitioners specialized in epilepsy. Another way to use the application might be to let patients and their relatives score the manifestations in advance of the consultation so that the physician can focus on most relevant details during the consultation.

In this study the DRFs were developed for epileptic seizures, but these frames also can be developed for other medical domains but consensus on the terminology of the disease classes and the disease manifestations is required.

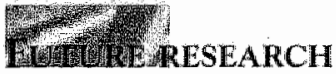

As mentioned in chapter 5 the first axis of "A proposed diagnostic scheme for people with epileptic seizures and with epilepsy" contains a list of ictal phenomena for describing seizure semiology, called the Glossary of Descriptive Ictal Terminology [13]. It might be useful to harmonize the Glossary and our list of manifestations into one glossary. The concepts we used to describe the manifestations are comparable with the concepts used in the Glossary alrhough not all concepts used in the Glossary and our list of manifestations can be translated one to one. E.g. in the Glossary the term 'dyscognitive" is used to indicate a disturbance of cognition, while on our list of manifestations we used the concept 'consciousness' which refers to the degree of awareness and/or responsiveness of the patient to externally applied stimuli. In the harmonization process one needs to decide whether these terms refer to the same (or sufficiently similar) concept and when (subtle) differences exist whether these differences are important or not. The risk of introducing new concepts like 'dyscognitive' is that physicians are not familiar with the terminology and therefore do not use the term or interpret the term wrongly. This can be eliminated by providing clear definitions of the terms.

This joint glossary can be used for describing seizure types in a structured way so real-life patient data can be collected uniformly in a database which makes the data more valuable for research. When many patients are included in the database the averaged conditional probabilities in the DRFs, at the moment based on frequency estimates of experts, can be updated. 


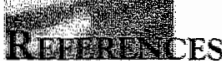

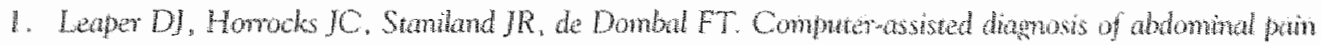
wsing "estimates" prosided by chicims. Br Med J 1972:41836):350.4.

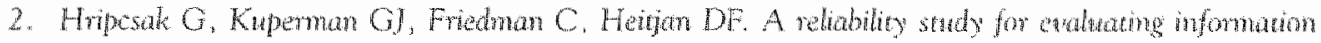
extraction from radiology reports. I Am Mod Infom Assoc 1999;6(2):143-50.

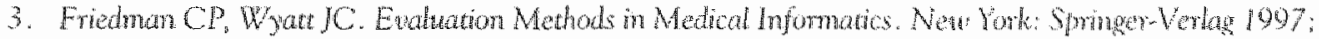
Chapter 5

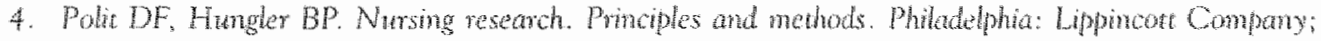
1995; Chapter 9

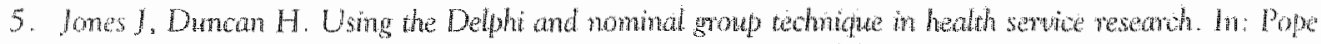

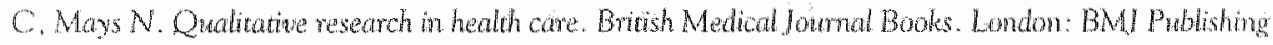
Group $2000 ; 40.49$.

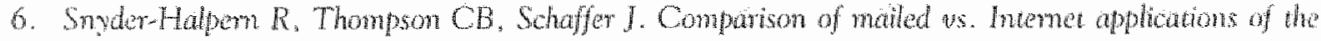
delphi technigue in chical infomatics reseath. Proc AMIA Symp 2000:809-13.

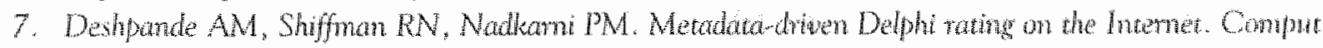
Methods Programs Biomed 2005;77(1):49-56.

8. Kors JA. Sittig AC, wan Bemmel JH. The Delphi method to waldate diagnostic lonowledge in computerized ECG interpratation. Method Inf Med 1990:29(1):44-50

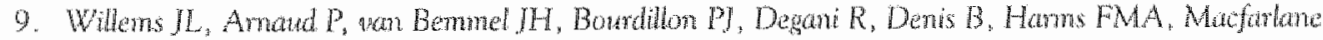
PW, Mazroca G, Meyer I, Risema wan Eck HJ Robles de Medina EO, Zywet C. Bstablishment of a reference library for ewalwating computer ECG measmement programs. Comput Biomed Res $1985 ; 18(5): 439-57$

10. Kong A, Bamet GO, Mosteller F, Youtz C. How medical professionals avaluate expressions of probability. N Engl J Med 1986;315(12):740:4.

11. Kortinen L, Pienla T. Peltola I, Nisila M, Keranen T, Toumen T, Petranek ES, Fray H. Euduation of Eplepsy Experta decision support system. Compu Methods Prangrams Biomed 1994;45(3):223-31.

12. Spizer $K$, Thie A, Caplan LR, Kunte $K$. The MICROSTROKE expent system for stroke type diagnosis. Sroke $1989 ; 20(10): 1353-6$

13. Engel J Ir. A proposed diagnostic schema for people with epileptic seitures and with apdlapsy: report of the ILAE Task Force on Classification and Teminology. Eprapsia 2001:42(6):796-803. 


\section{APPENDIX I}

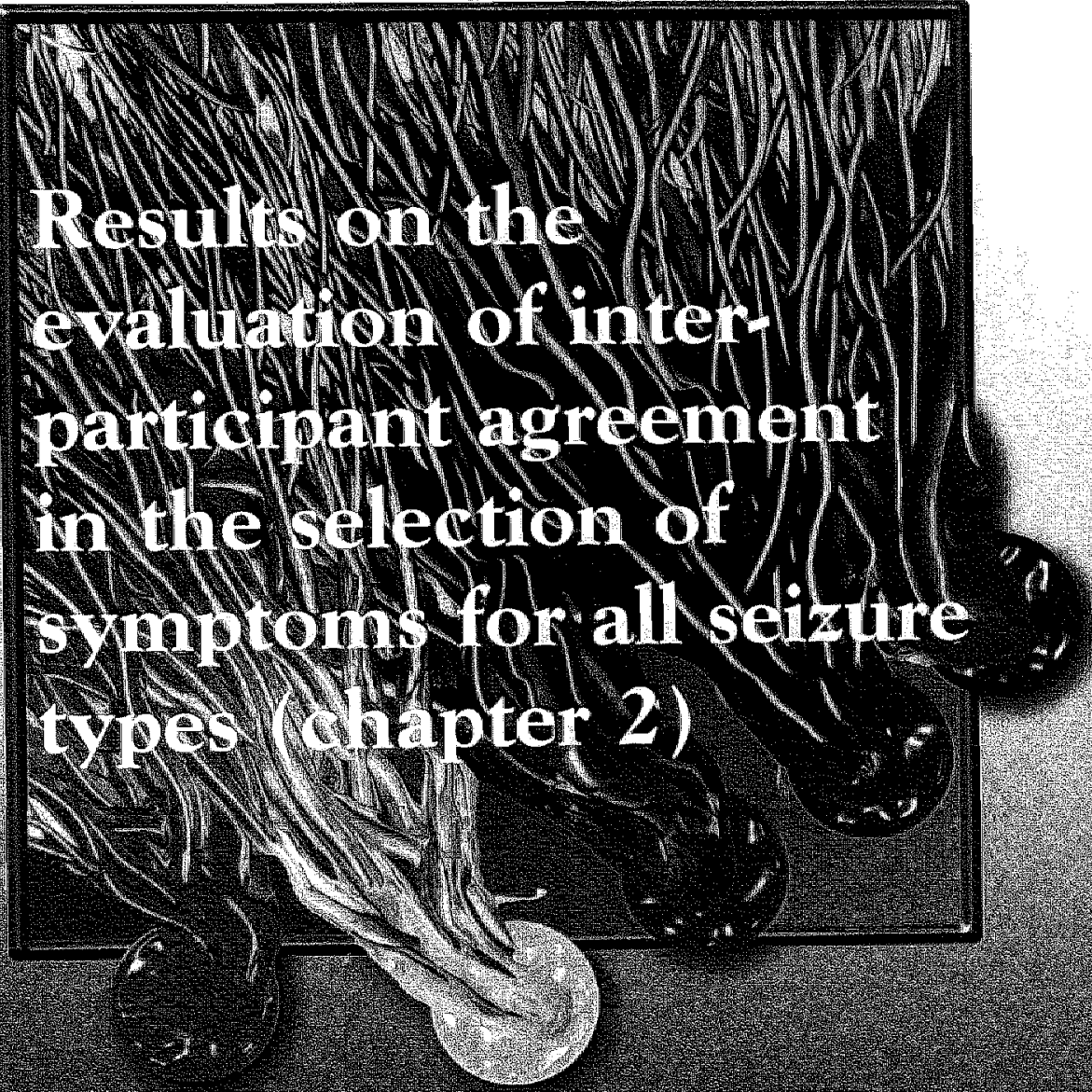



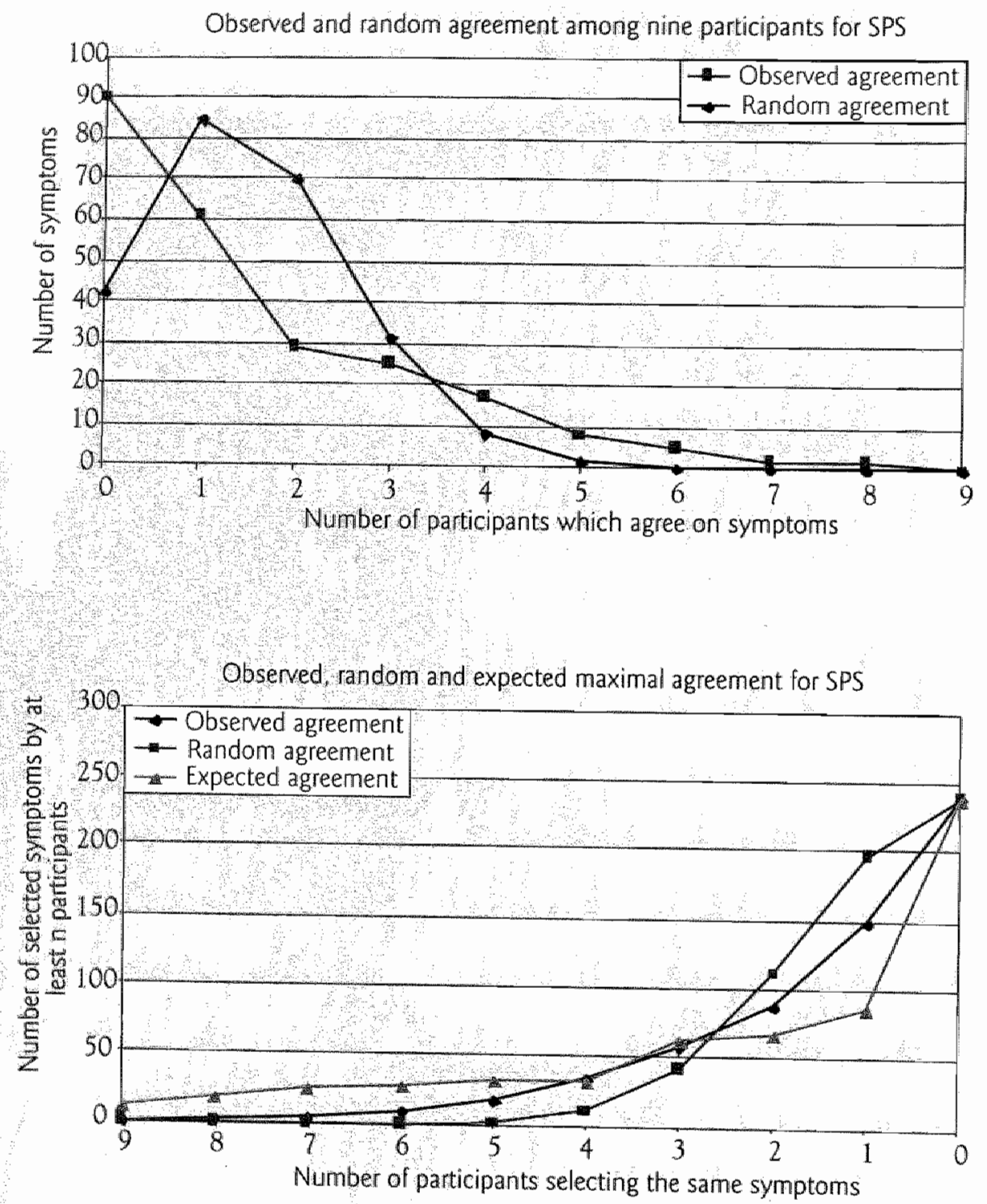

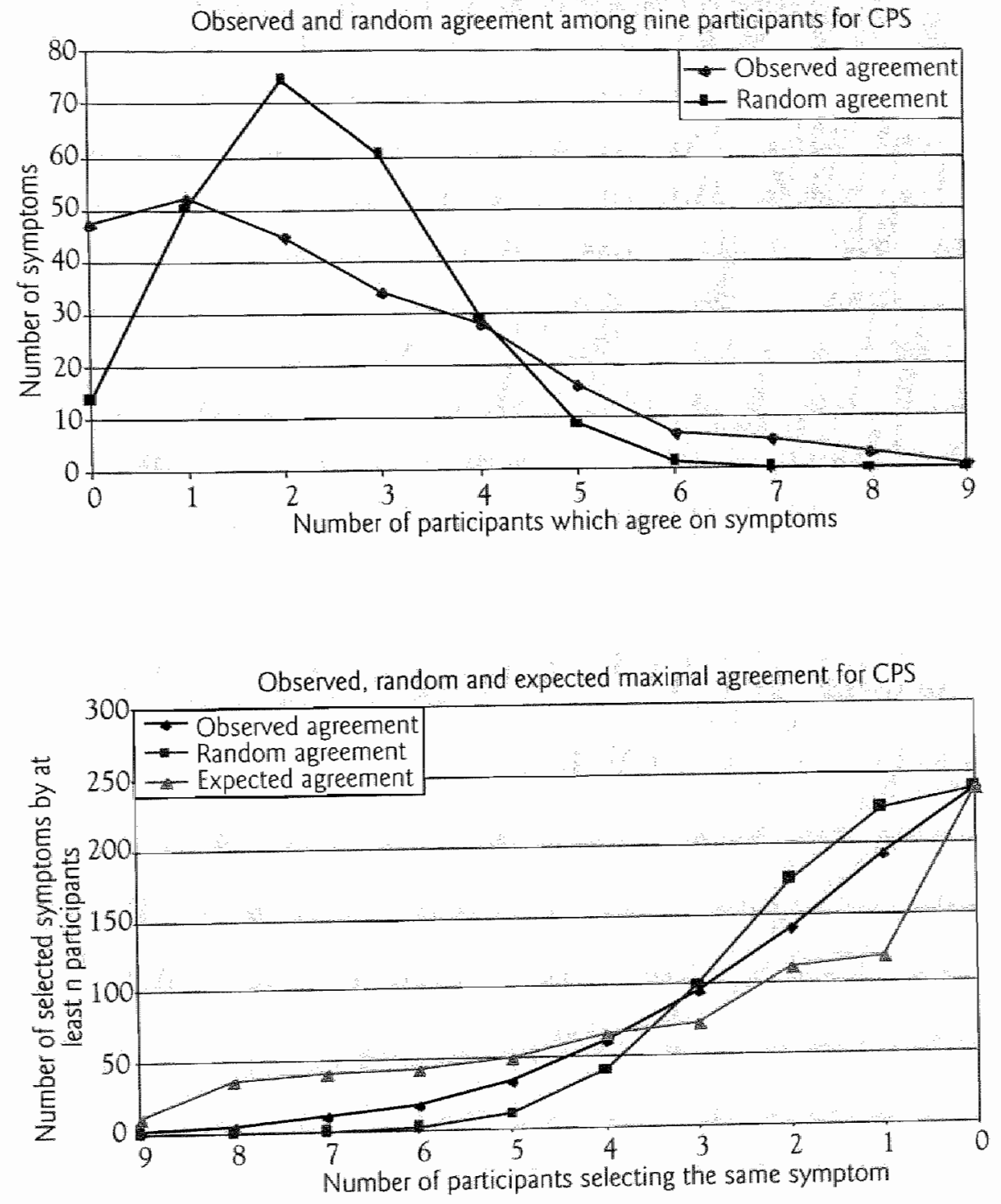

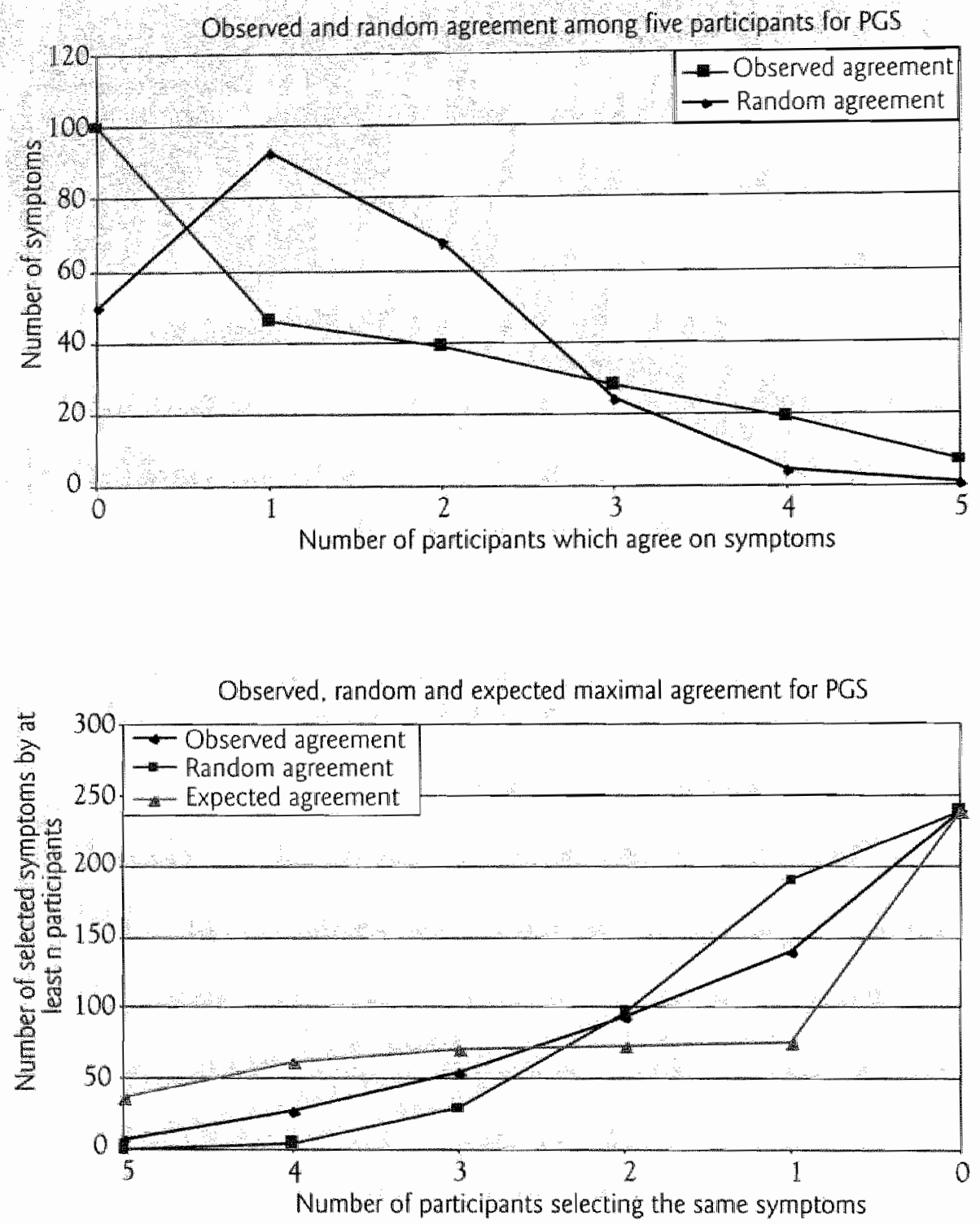
Observed and random agreement among five participants for SCS

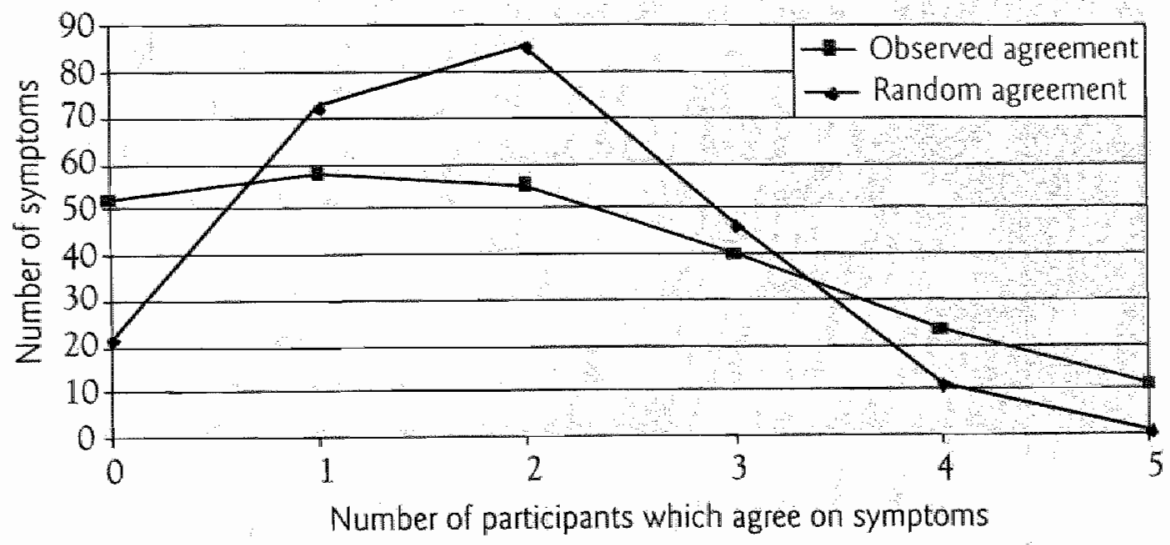

Observed, random and expected maximal agreement for SGS
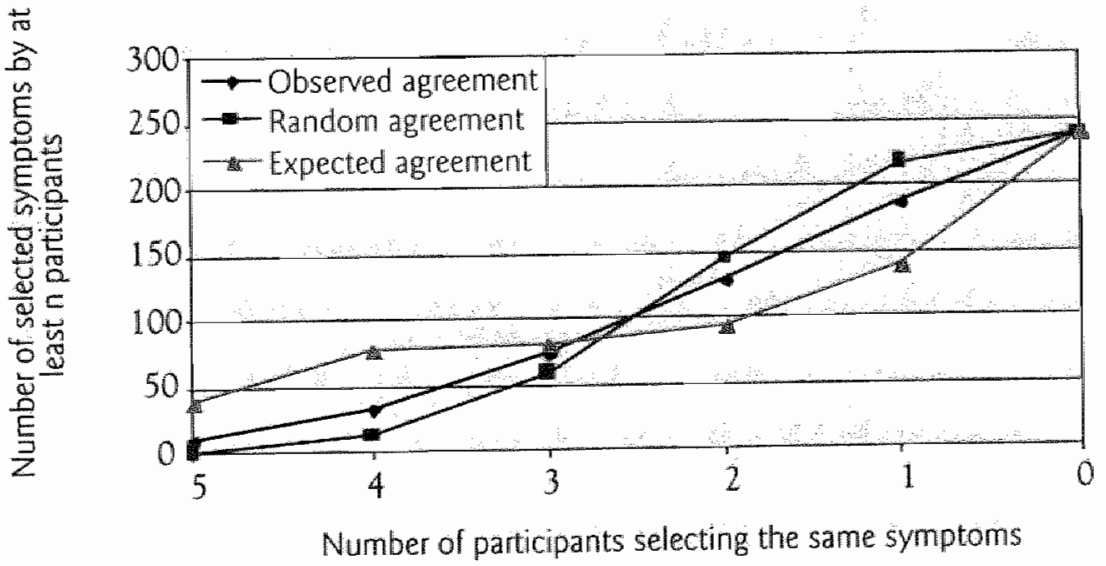
Observed and random agreement among six participants for AS

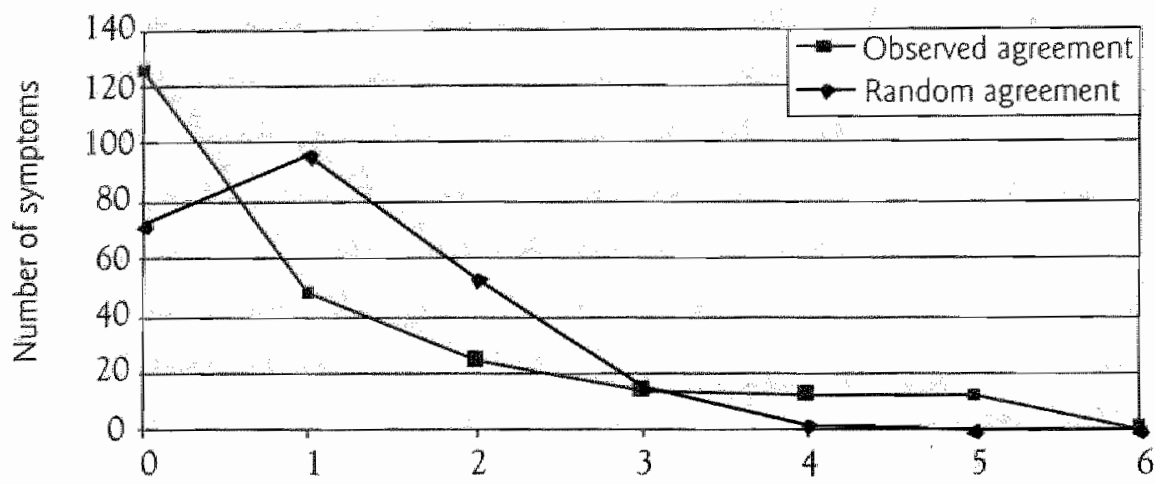

Number of participants which agree on symptoms

Observed, random and expected maximal agreement for AS
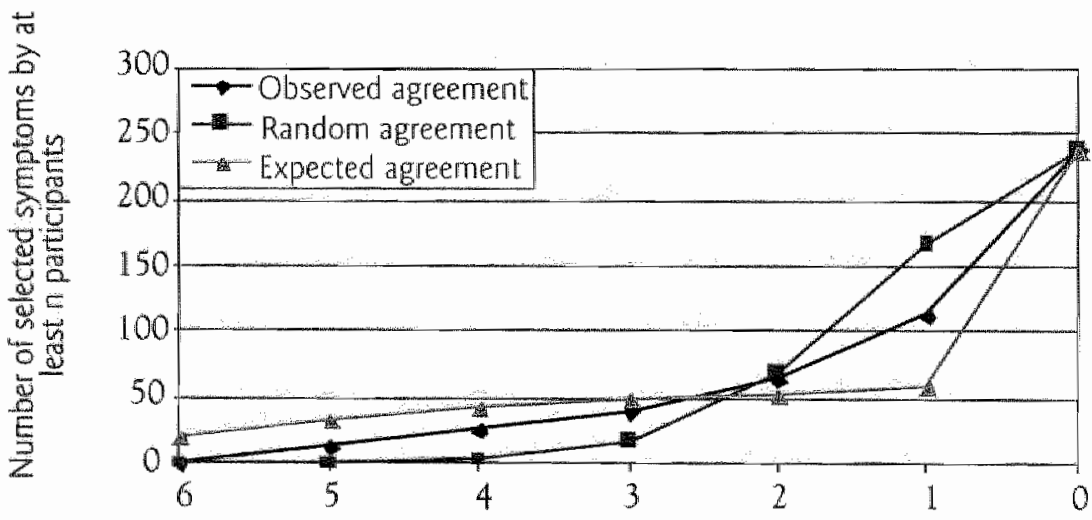

Number of participants selecting the same symptoms 
Observed and random agreement among six participants for MS

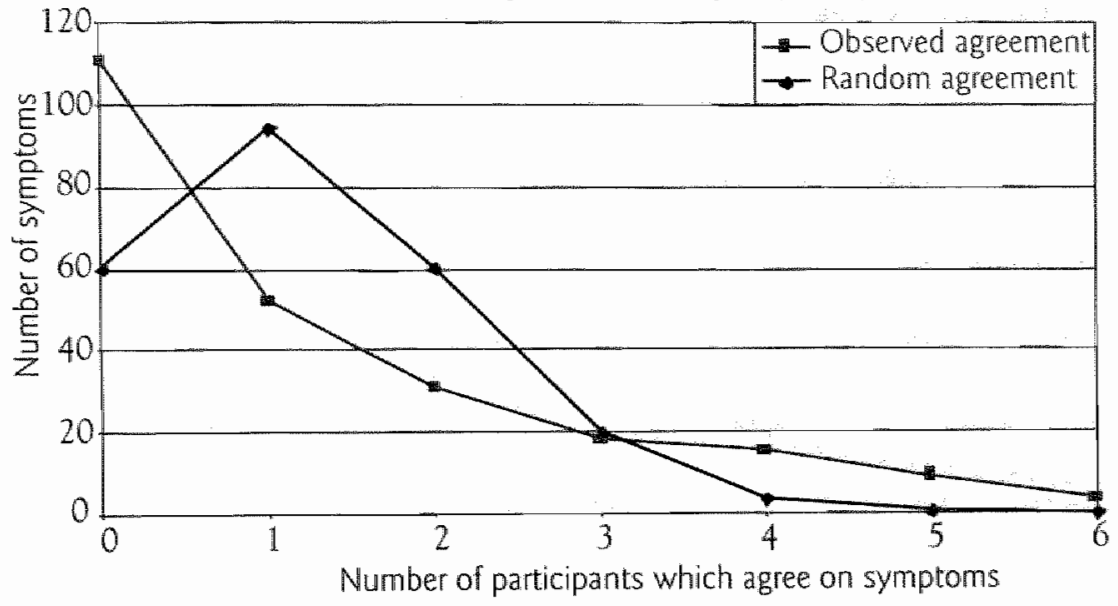

Observed, random and expected maximal agreement for MS

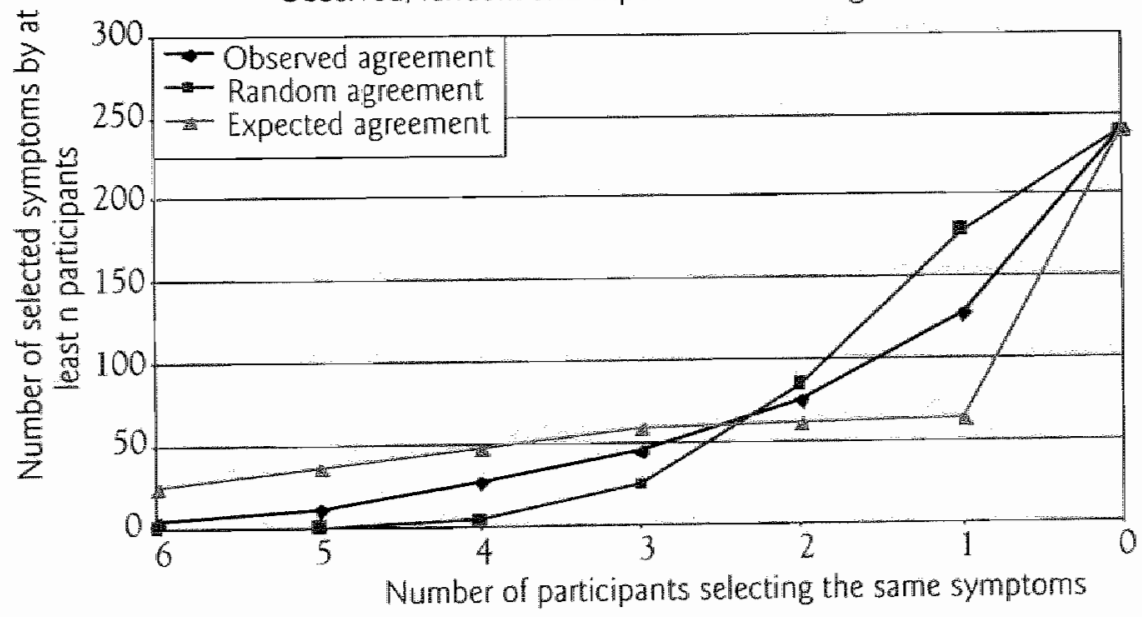



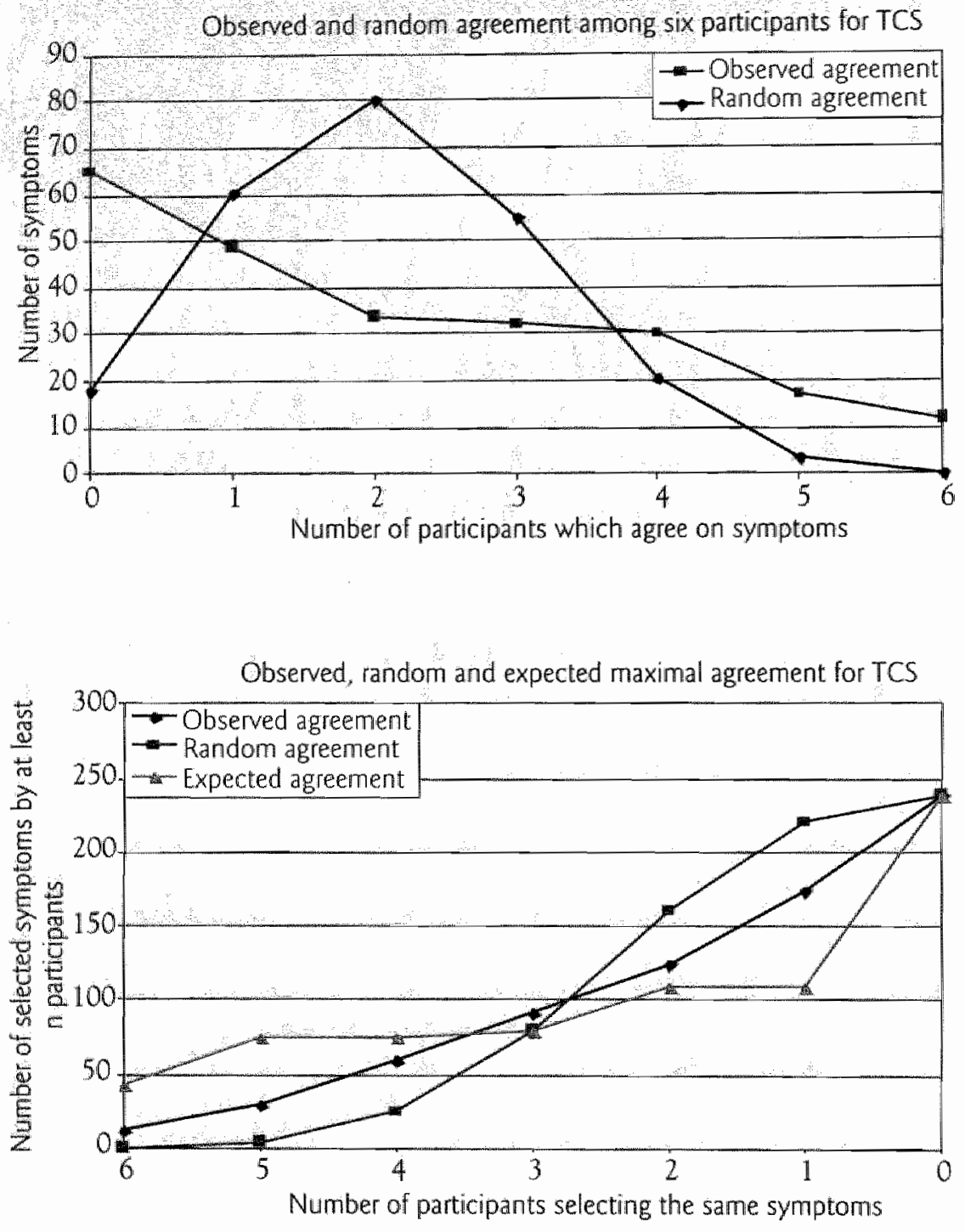


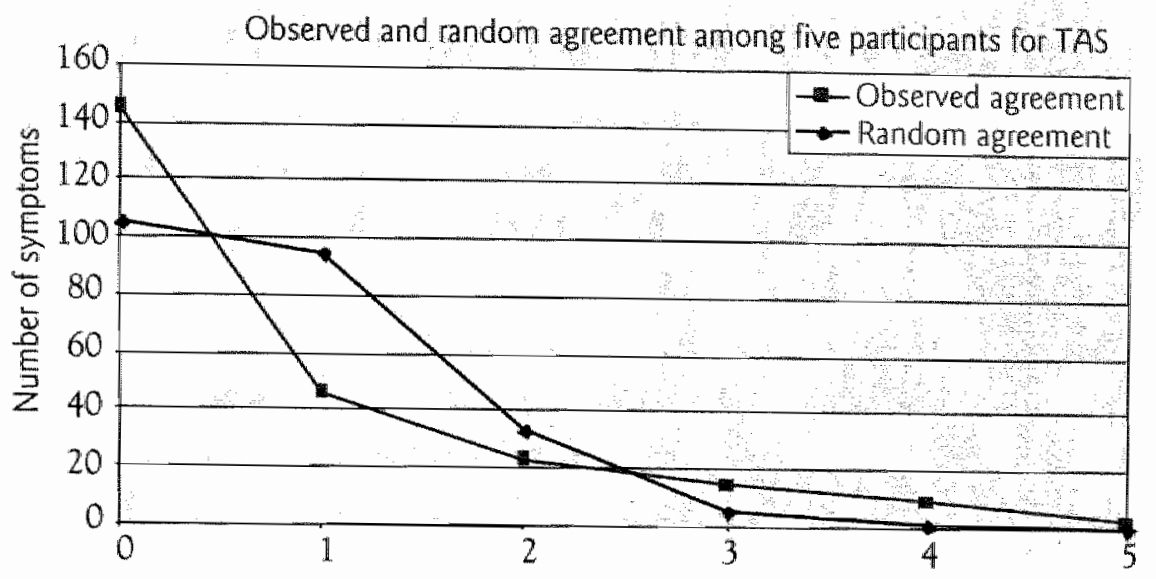

Number of participants which agree on symptoms

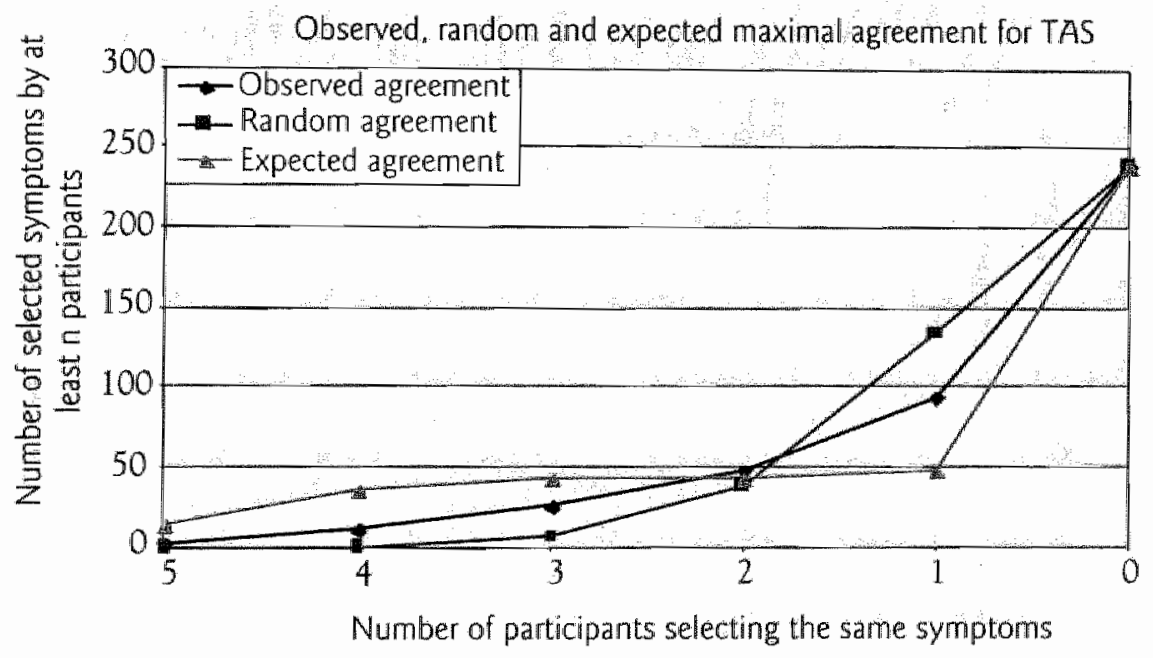



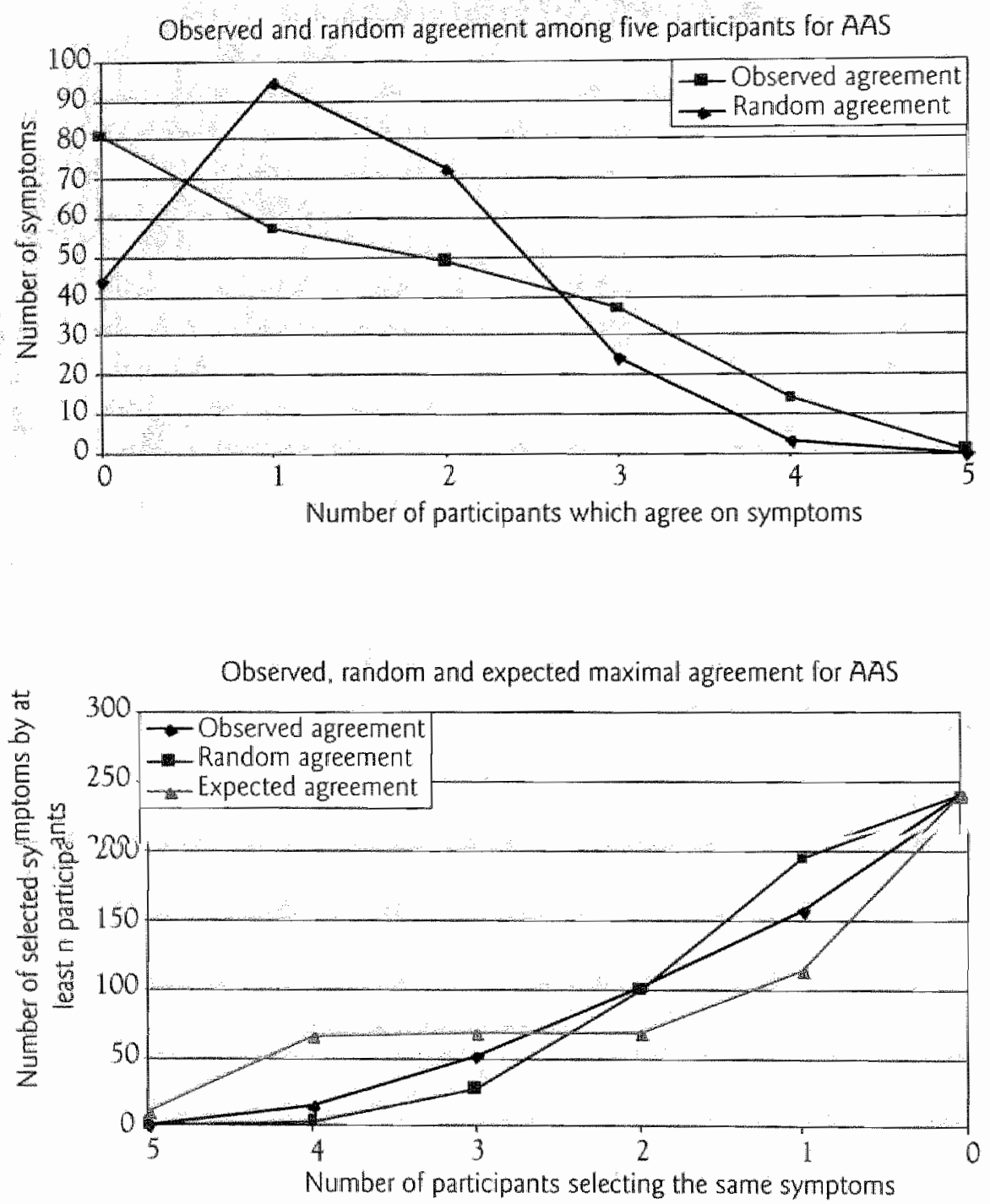


\section{APPENDIX II}

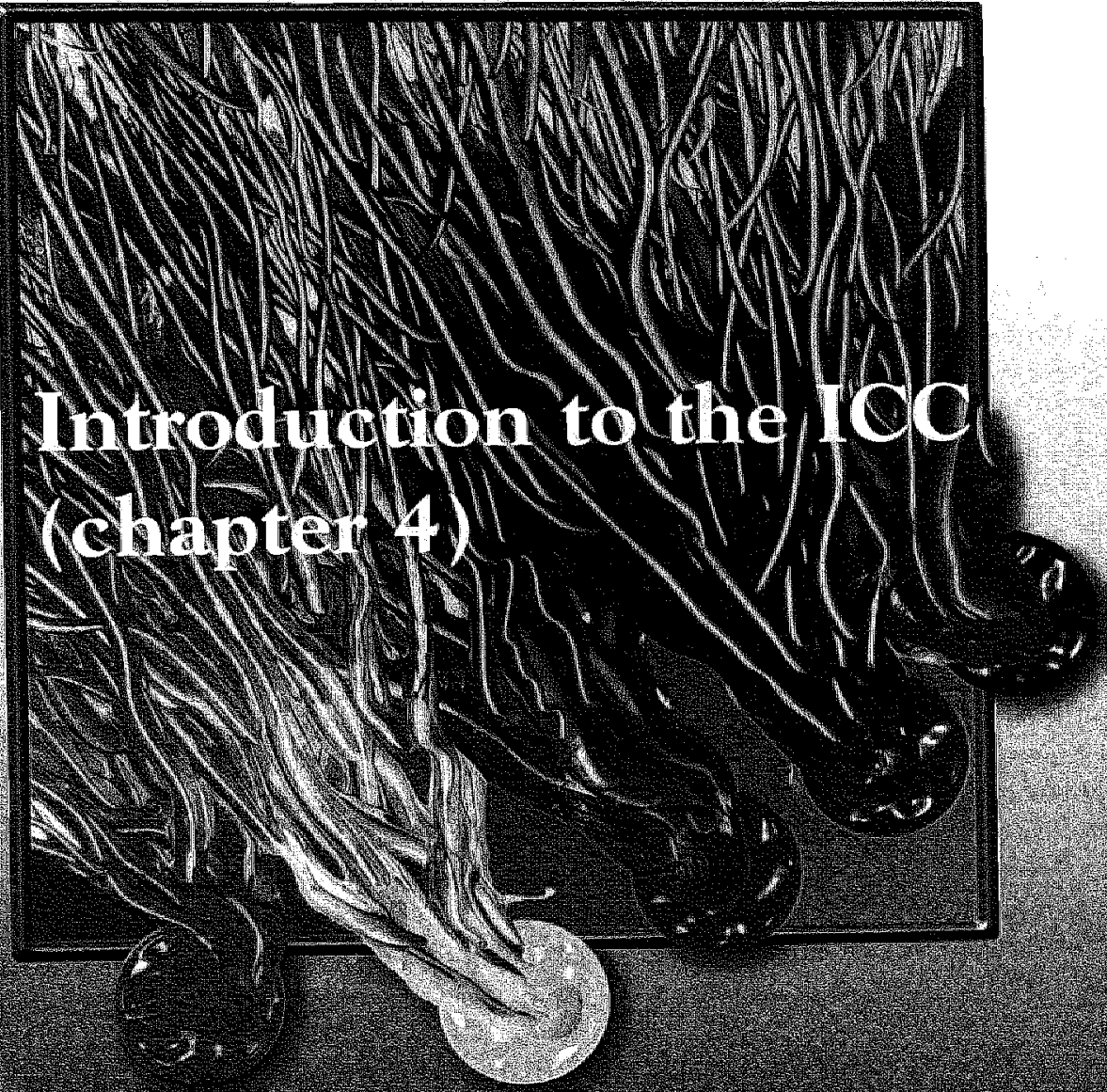




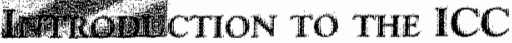

In this study we determine the frequency of occurrence of all relevant manifestations in each of six seizure rypes. Estimates of these frequencies are provided by participants in the research project. The frequency of occurrence for each manifestation is then obtained by averaging the frequency estimates of the individual participants for each seizure type. If the participants are truly consistent their estimates will be exactly the same. Usually participants make random errors when estimating frequencies. In addition the participants may differ systematically. Estimates are reliable when the spread among the values of the participants is small. The more reliable the estimates, the smaller the random and systematic errors. The extent of the reliablity is expressed by the inter-rater intraclass correlation coefficient (ICC), as used in the behavioral sciences to examine the dependability of behavioral measurements [1]. The model used to describe the frequency assessment of each participant is:

$x_{i j k}=\mu_{k}+a_{i k}+b_{j k}+w_{i j k}$ where

$\mu_{k}$ is the population mean for all observations in seizure type $k$,

$x_{i j k}$ is the frequency of occurrence of manifestation $i$ as estimated by participant $j$ for a seizure type $k_{\text {, }}$

$a_{i k}$ is the true frequency of occurrence of a manifestation $i$ in seizure type $k$,

$b_{j k}$ is the systematic error made by participant $j$ in seizure type $k$,

$w_{i j k}$ is the random error made by participant $j$ in estimating the frequency of occurrence of a manifestation $i$ in a seizure type k.

Note that the data for each seizure type $k$ can be represented by a table with I rows (I is the number of manifestations of which the frequency of occurrence is estimated) and $]$ columns (the number of participants). The cell belonging to the $\mathrm{i}^{\text {th }}$ row and the $\mathrm{j}^{\text {th }}$ column of the table for seizure type $k$ contains the frequency estimate $x_{i j k}$.

The total variance of the I J estimates can be determined from the table. The intraclass correlation coefficient (IOC) is defined as the fraction of this total variance (which is equal to the sum of the true variance and the wariances due to random and systematic errors) that is caused by the true differences in frequency of occurrence of the manifestations (true variance) [2]. An ICC of one indicates that the participants make neither systematic nor random errors, so that all frequency estimations $x_{i j k}$ are correct.

Two types of ICQ can be discerned: the intra-rater and the inter-rater 1CC. The intratater ICC measures the average reliability of the estimates of individual participants whereas the inter-rater $10 \mathrm{C}$ measures the reliability of the estimates, averaged over all participants. The inter-rater ICC will increase with the number of participants, whereas the intra-rater ICC does not.

In addition there are two approaches that can be used in the calculation of the ICC (whether intra- or intetrater ICC). In the consistency approach the ICC is defined as the ratio of the rue vatiance and the total variance of the data (consisting of true and wadom variance, but excluding the variance due to systematic differences between participants). In this approach it is assumed that the experts are consistent, i.e. they ether do not differ systematically or the systematic differences do not influence the results. This approach can be used when for example the goal would be to rank the manifestations according to their frequency of occurrence (systematic differences appear as a constant contribution in all frequency estimates and therefore do not influence the ranking itself). 
In the second approach, the absolute agreement approach, the observer variance due to systematic differences between the participants is also included in the total variance [2], Since in this study we are interested in the true values of the frequency of occurrence we have to use the second approach.

Cohen's kappa is often used to determine inter-observer agreement. Cicchetti et al. [3] state that "when the purpose of studying observer variability is to reduce it rather than to quantify its magnitude kappa is not satisfactory, because the value of kappa will be influenced by the distribution of marginal totals". The ICC gives us the possibility nor only to determine the reliability of the data but also allows us to determine how many observers are needed to obtain the specified ICC value.

Note that the ICC is an aggregate measure, taking the variability of all individual manifestations into account. This means that some individual manifestations still may show an appreciable variability. It is suggested that an ICC larger than 0.7 indicates that the reliability of the set of manifestations on the whole is good, some may be less reliable and some more reliable. If one wants to have reliable frequency estimates for all manifestations separately, a higher ICC is needed. Friedman and Wyatt indicate that 'an ICC of 0.9 or above is often necessary when the concern is assignment of scores to individual objects with a high degree of precision' [4].

In practice usually there is no information about the variability of estimates or judgements in a certain domain so that one does not know beforehand how many participants are needed to obtain results with a high enough intra- or inter-rater ICC. Therefore the actual study (also called demonstration study) is preceded by a so-called measurement study to measure the extent of random and systematic errors in the domain. From the results of the measurement study an intra- or inter-rater ICC can be determined. By using the so-called Spearman-Brown prophecy formula one can then determine the number of participants needed in the demonstration study to obtain the value of the ICC that one wants. The Spearman-Brown formula for the inter-rater ICC is given by:

$1 \mathrm{ICC}_{\mathrm{n}}=\frac{q^{*} \mathrm{ICC}_{\mathrm{k}}}{1+(q-1) \mathrm{ICC}_{\mathrm{k}}} \quad$ where

$1 \mathrm{ICC}_{n}$ is the inter-tater intraclass correlation coefficient with $n$ observers,

$1 C C_{k}$ is the inter-rater intraclass correlation coefficient with $k$ observers obtained vis the measurement study,

$q$ is $n / k$.

As can be seen from the formula the data from the measurement study ate needed to determine the number of participants needed for a certain value of the ICC. One now can carry out the demonstration study with the number of participants as determined via the Spearman-Brown prophecy formula. The $1 \mathrm{CC}$ can be calculated from the data of the demonstration study to determine whether the participants in the demonstration study were different from the participants in the measurement study. In this study the measurement study was carried out with the Dutch participants. When $n$ is taken as 1 , the intrarater ICC is obtained. An ICC for one observer can only be derermined when a measurement study with more observers has been carried out. 
Appendix II 


\section{2(1)}

1. Shavelson RJ, Webb NM, Rothey GL. Generalizabihy thory. Am Psychol 1989:4461922.932.

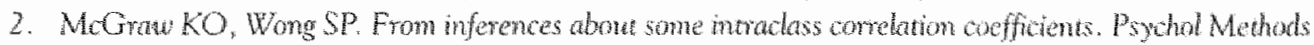
$1996,1(1): 30+46$.

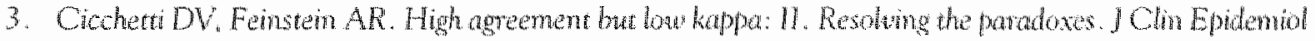
$1990,43(6) .551-8$

4. Fredman CP, Wyat JC. Evaluaion Methods in Medical momatics. New York. Springer Verag; 1997. 



\section{APPENDIX III}

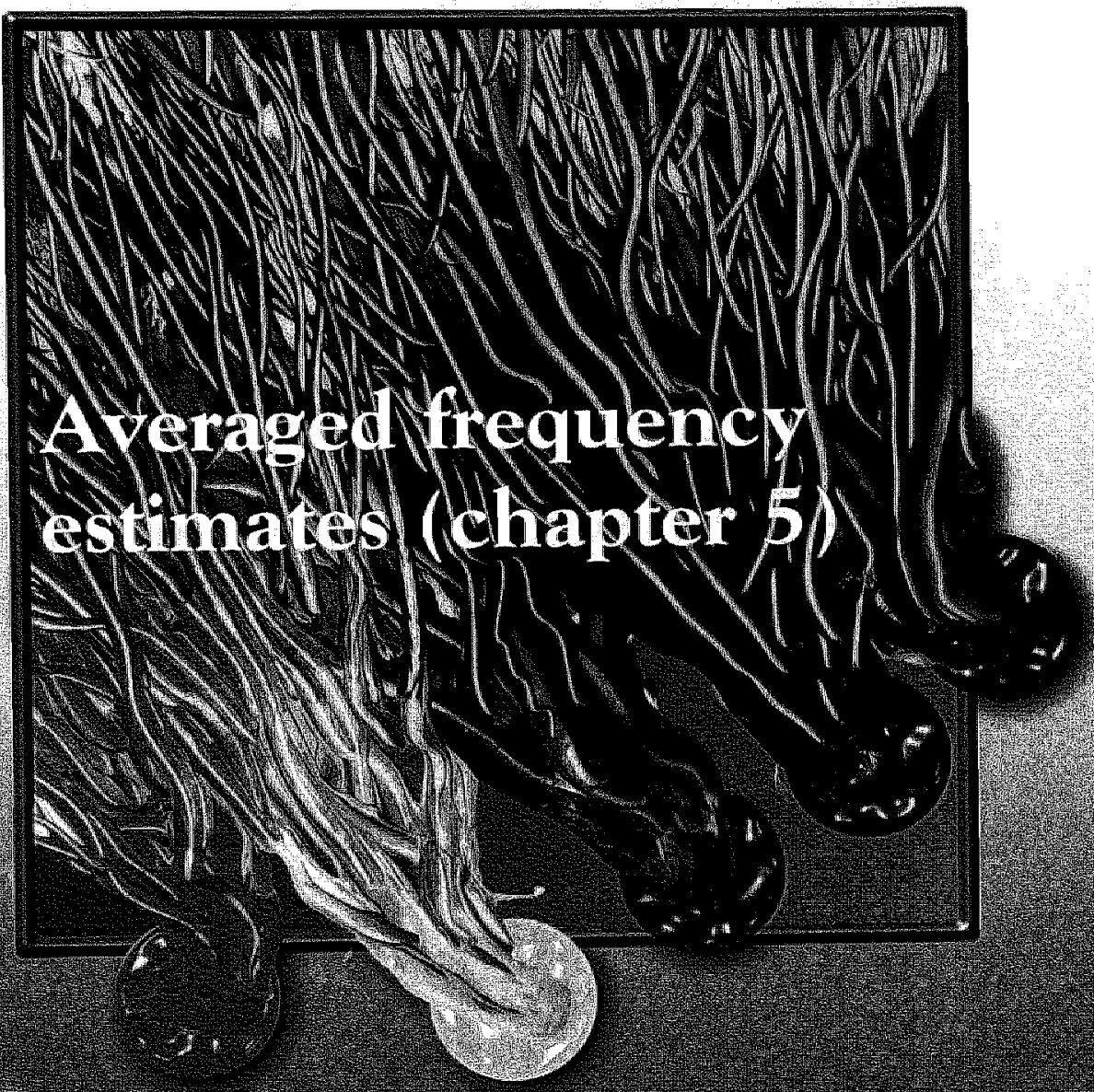




\section{VIN III}

Averaged frequency estimates $-p\left(s_{i} / S_{i}\right)$ - for each manifestation in each seizure type

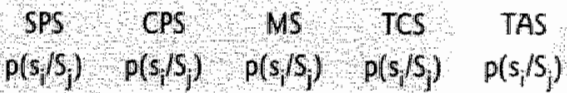

Seizure tharacteristics

Onset of seizure

Difficult to identify

$\begin{array}{lllll}0.20 & 0.16 & 0.11 & 0.05 & 0.20 \\ 0.16 & 0.33 & 0.04 & 0.09 & 0.07 \\ 0.26 & 0.30 & 0.13 & 0.28 & 0.04 \\ 0.37 & 0.22 & 0.72 & 0.58 & 0.69\end{array}$

Cessation of seizure"

$0.21 ? 0.24 \quad 0.05 \quad 0.09 \quad 0.18$

Slowly gradual

01600

0,31 ? 0.2

Rapidly gradual

0.33

$0.28 \div 0.25$

0.18

0.04

Sudden

01

0.6

0.36

0.04

Duration of selzure

$<10$ seconds

10 seconds 30 seconds

0,10

$0.61 \smile 0.06$

0.39

03

018

0.13

0.15

0.37

$>30$ seconds 3 minutes

0,117

0,34

0.1

0,39

0.13

$>3$ minutes

01

Vairiable

0.22

0.17

0.06

0.23

0.07

Time of day seizure occurs:

Around awakening from sleep

011

02

0.08

0.17

0.05

Around going to sleep

During sleep

0.13

0.08

$0,3 4 \longdiv { 0 . 2 5 }$

0.12

At onher times

0.12

0.14

0.14

0.14

0.08

Around daytime

0.6

0.15

0.09

0.17

0.03

loregular

Course of the seizure*

022

0,17

0,12

0.15

0.22

026

0.23

$0 / 8$

013

0.44

0.26

0.22

014

0.15

0.12

Partilat at onset

Generalized at onset

Partial at onset, then secondary generalized

$\begin{array}{lllll}0.65 & 0.60 & 0.23 & 0.22 & 0.05 \\ 0.06 & 0.05 & 0.59 & 0.54 & 0.90 \\ 0.29 & 0.35 & 0.5 & 0.25 & 0.05\end{array}$

\section{Preilctal phenomena}

Prodrone

Ml-defined vague feeling

Myoclonic movements

Mood changes

$\begin{array}{lllll}0.37 & 0.60 & 0.10 & 0.44 & 0.11 \\ 0.11 & 0.16 & 0.18 & 0.34 & 0.18 \\ 0.27 & 0.46 & 0.08 & 0.32 & 0.05 \\ 0.14 & 0.25 & 0.16 & 0.37 & 0.08\end{array}$




$$
\begin{array}{ccccc}
s P S & C P S & M S & T C S & T A S \\
p\left(s_{i} / S_{j}\right) & p\left(s / S_{j}\right) & p\left(s_{i} / S_{j}\right) & p\left(s_{i} / S_{j}\right) & p\left(s_{i} / S_{j}\right)
\end{array}
$$

Motor signs and symptoms

Automatisms

Ambulatory

Gestural

Mimetic

Oro-alimentary

Verbal

Movements

Clonic movements

Tonic movemerits

Atonic movements

Myoclonic movements

Bodypart distribution*

Bilateral

Unilateral

Bodypart inwolvement

Face

Arms

Hands

Legs:

Trunk

Muscle (isolated)

Muscle group (isolated)

Muscle contractions

Symmetric*

Asymmetric*

Regular*

Irregular*

Generalized

Rhythmic

Muscle spasms

Axial

Axorhizomelic

Global

$\begin{array}{lllll}0.18 & 0.47 & 0.09 & 0.07 & 0.11 \\ 0.18 & 0.66 & 0.05 & 0.09 & 0.17 \\ 0.22 & 0.45 & 0.05 & 0.10 & 0.14 \\ 0.22 & 0.69 & 0.08 & 0.12 & 0.27 \\ 0.20 & 0.46 & 0.05 & 0.16 & 0.08\end{array}$

$0.64 \quad 0.28 \quad 0.30 \times 0.91 \quad 0.24$

$\begin{array}{llllll}0.43 & 0.52 & 0.1150 & 0.89 & 0.18\end{array}$

$0.20 \quad 0.24 \quad 0.10 \quad 0.16 \quad 0.05$

$\begin{array}{lllll}0.27 & 0.25 & 0.92 & 0.4 & 0.23\end{array}$

$\begin{array}{llllll}0.18 & 0.44 & 0.61 & 0.84 & 0.94\end{array}$

$\begin{array}{lllll}0.82 & 0.56 & 0.39 & 0.16 & 0.06\end{array}$

$\begin{array}{llllll}0.57 & 0.70 & 0.56 & 0.83 & 0.24\end{array}$

$\begin{array}{llllll}0.52 & 0.67 & 0.78 & 0.89 & 0.24\end{array}$

$\begin{array}{lllll}0.50 & 0.68 & 0.55 & 0.87 & 0.20\end{array}$

$\begin{array}{llllll}0.41 & 0.42 & 0.50 & 0.89 & 0.14\end{array}$

$\begin{array}{lllll}0.27 & 0.30 & 0.46 & 0.82 & 0.05\end{array}$

$\begin{array}{lllll}0.17 & 0.11 & 0.19 & 0.10 & 0.05\end{array}$

$\begin{array}{llllll}0.31 & 0.17 & 0.31 & 0.10 & 0.08\end{array}$

$\begin{array}{lllll}0.09 & 0.16 & 0.59 & 0.77 & 0.88\end{array}$

$\begin{array}{lllll}0.91 & 0.84 & 0.41 & 0.23 & 0.12\end{array}$

$\begin{array}{lllll}0.46 & 0.49 & 0.51 & 0.67 & 0.81\end{array}$

$\begin{array}{lllll}0.54 & 0.51 & 0.49 & 0.33 & 0.19\end{array}$

$\begin{array}{lllll}0.07 & 0.21 & 0.49 & 0.80 & 0.18\end{array}$

$\begin{array}{lllll}0.36 & 0.32 & 0.33 & 0.71 & 0.31\end{array}$

$\begin{array}{lllll}0.24 & 0.17 & 0.31 & 0.46 & 0.11\end{array}$

$\begin{array}{lllll}0.27 & 0.18 & 0.22 & 0.26 & 0.05\end{array}$

$\begin{array}{lllll}0.27 & 0.27 & 0.23 & 0.82 & 0.05\end{array}$ 


$\begin{array}{ccccc}\text { SPS } & \text { CPS } & \text { MS } & \text { TCS } & \text { TAS } \\ p\left(s_{i} / S_{j}\right) & p\left(s_{j} / S_{j}\right) & p\left(s_{i} / s_{j}\right) & p\left(s_{i} / S_{j}\right) & p\left(s_{i} / S_{j}\right)\end{array}$

Changes in postural tone

Bowing/bending movements

Head turned nito a particular direction

$0.27-0.37$

03

0.21

0.08

Limbs held stiff in a particular position

036

0,6

0.19

039

0.05

$0.22 \quad 0.52$

0.14

$0: 52$

0.05

Loss of tone in neck

$0.08 \quad 0.14$

0.14

0.10

0.08

Loss of tone in trunk

Nodding of head

$0.08 \quad 0.14$

0.18

0.10

0.08

Propulsion of hand held objects

$0.17 \quad 0.22$

0.35

0.10

0.20

$0.13 \quad 0.19$

0.51

0.05

0.08

Rotation of whole body

$0.25 \quad 0.37$

0.13

0.09

0.05

Sagged at knee

$0.12 \quad 0.18$

0.29

0.05

0.05

Sudden fall

0.15

0.21

0.35

0.70

0.08

Abnormal eye movements*

Eyes look ahead and do rot follow

0.06

Eyes are turned upwards

$0.06-0.29$

0.07

0.06

0.58

Eyes are turned downwards

$0.06 \quad 0.11$

0.09

0.24

0.17

Eyes are turned left

$0.04 \quad 0.05$

0.05

0.04

0.05

Eyes are turned right

0.17

0.23

0.12

0.16

0.07

No abnormal eye movements

$0.17 \quad 0.23$

0.12

0.16

0.07

$0.51 \quad 0.10$

0.54

0.35

0.07

Non-motor symptoms

Prodrome

Epigastric

Gustatory

0.67

0.05

0.37

0.05

0.26

0.48

0.05

0.28

0.05

Visual

0.30

0.45

0.05

0.28

0.05

Auditory

$\begin{array}{lll}0.25 & 0.41 & 0.05\end{array}$

0.25

0.05

Ollactory

0.26

0.41

0.05

0.23

0.05

Somatosenisory symptoms

$0.43 \quad 0.40$

0.07

0.27

0.05

Special sensory symptoms

$0.38 \quad 0.46$

0.05

0.27

0.05

Experiential symptoms

0.30

0.45

0.05

0.25

0.05

\section{Consciousness}

Influence of seizure on angoing activity*

No loss

$0.52 \quad 0.05$

0.47

0.05

0.04

Retains some lunctioning

$0.33 \quad 0.36$

0.28

0.09

0.15

Stops roomal ongoing activity

Influence of seizure on response to stimuli*

$0.15 \quad 0.5$

0.25

0.85

0.81

Nó influence

Retains some awareness

$\begin{array}{lllll}0.55 & 0.04 & 0.50 & 0.03 & 0.03\end{array}$

Unresponsive to spoken words and visual stimuli

$0.35 \quad 0.30$

0.29

0.05

0.12

0.07

0.45

0.13

0.47

0.60

Unresponsive to painful stimuli

0.04

0.21

0.07

0.46

0.25 


$\begin{array}{ccccc}S P S & C P S & M S & T C S & T A S \\ p(s / 5) & \rho\left(s / S_{i}\right) & \rho\left(s / s_{i}\right) & p\left(s_{i} / S_{j}\right) & p\left(s_{i} / s_{j}\right)\end{array}$

Speed of loss of consciousness*

No loss

Abrupt sudden loss of cons.

Gradual loss of consciousness

$\begin{array}{lllll}0.90 & 0.06 & 0.68 & 0,04 & 0.05 \\ 0.05 & 0.33 & 0,22 & 078 & 0.83 \\ 0.05 & 0.62 & 0,10 & 018 & 0.12\end{array}$

Somatic reactions

Autonomic symptoms

Change of blood pressure

Change of heartipulse rate

Change of respiratory rate

Flushing

Paillor skin

Borborygmi

Epigastric sensation (see Aura)

Vomiting

Urine incontinence

Pupillary dilatation

Piloerection

Increased salivation

Sweating

Vertigo

Cyanosis:

$\begin{array}{lllllll}0.3 & 0.34 & 007 & 0.81 & 011\end{array}$

$\begin{array}{lllll}0.40 & 0.55 & 0.08 & 0.81 & 0.11\end{array}$

$\begin{array}{lllll}0.27 & 0.53 & 0.10 & -0.8 & 0.14\end{array}$

$\begin{array}{llllll}0.28 & 0.41 & 0.05 & 0.58 & \because & 0.14\end{array}$

$\begin{array}{lllll}0.29 & 0.47 & 0.12 & 0.40 & 0.14\end{array}$

$\begin{array}{llllll}0.23 & 0.36 & 0.05 & 0.18 & 0.05\end{array}$

$\begin{array}{lllll}0.38 & 0.67 & 0.05 & 0.28 & 0.05\end{array}$

$\begin{array}{llllll}0.11 & 0.35 & 0.07 & 0.31 & 0.05\end{array}$

$\begin{array}{lllll}0.08 & 0.26 & 0.18 & 0.70 & 0.08\end{array}$

$\begin{array}{lllll}0.20 & 0.45 & 0.08 & 0.70 & 0.18\end{array}$

$\begin{array}{lllll}0.18 & 0.29 & 0.07 & 0.23 & 0.08\end{array}$

$\begin{array}{lllll}0.18 & 0.46 & 0.07 & 0.44 & 0.08\end{array}$

$\begin{array}{lllll}0.18 & 0.36 & 0.07 & 0.44 & 0.08\end{array}$

$\begin{array}{lllll}0.19 & 0.25 & 0.05 & 0.12 & 0.05\end{array}$

$\begin{array}{lllll}0.05 & 0.15 & 0.05 & 0.51 & 0.05\end{array}$

Other somatic reactions

Tongue biting.

Vocalization (e.g. scream)

$\begin{array}{lllll}0.05 & 0.14 & 0.10 & 0.68 & 0.05\end{array}$

$\begin{array}{lllll}0.23 & 0.41 & 0.18 & 0.48 & 0.05\end{array}$

Post-ictal phenomena

Post-ictal altered behaviour

Confusion

0.10

0.72

0.13

$0.70 \quad 0.08$

Drowsiness

Stupor

Unaware of having had a seizure

0.16

0.59

0.13

$0.76 \quad 0.08$

0.05

0.23

0.13

$0.48 \quad 0.05$

Psychotic symptoms

0.08

0.59

0.22

$0.34 \quad 0.79$

0.08

0.23

0.05

$0.27 \quad 0.05$

Post-ictal headache*

No headache

Particular part of head

$\begin{array}{lllll}0.72 & 0.30 & 0.80 & 0.43 & 0.86\end{array}$

One side of head

0.09

0.04

$0.09 \quad 0.04$

Overall

0.07

0.24

0.04

$0.10 \quad 0.04$

0.12

0.12

$0.39 \quad 0.06$

Falling asleep posit-ictally*

No falling asleep

$\begin{array}{lllll}0.80 & 0.38 & 0.80 & 0.13 & 0.78 \\ 0.15 & 0.38 & 0.07 & 0.40 & 0.17 \\ 0.05 & 0.25 & 0.14 & 0.47 & 0.05\end{array}$

Sleeps $<30$ minutes

Sleeps $>$ I hour

0.05

0.20

0.06 


$\begin{array}{ccccc}s P S & C P S & M S & T C S & T A S \\ p\left(s / S_{j}\right) & p\left(s_{i} / s_{i}\right) & p\left(s_{i} / S_{j}\right) & p\left(s_{i} / S_{j}\right) & p\left(s_{i} / s_{i}\right)\end{array}$

Other postuctal phenomera

$\begin{array}{lccccc}\text { Dizziness } & 0.12 & 0.30 & 0.05 & 0.25 & 0.05 \\ \text { Muscle soreness } & 0.15 & 0.21 & 0.13 & 0.53 & 0.05 \\ \text { Speech disturbance } & 0.18 & 0.35 & 0.05 & 0.25 & 0.05 \\ \text { Paresis } & 0.25 & 0.22 & 0.12 & 0.25 & 0.05 \\ \text { Paralysis } & 0.16 & 0.14 & 0.07 & 0.20 & 0.05\end{array}$

\section{EEC-characteristics}

Characterization of discharges

spikes

Spikes and waves

Polyspikes and waves

Sharp waves

Slow waves

High woltage

Hemispheric involvement*

Unilateral

Bilateral other

Bilateral synchronous

Diffuse

Localization epileptic discharges ${ }^{*}$

Frontal

Central.

Parietal

Occipital

Temporal

Multifocal

Type ol propagation *

Primary generalized

Secondary generalized

EECu-background activity

Normat

Abnormalt

Regulart

Irregullar"*

Symmetrical ${ }^{*}$

Asymmetrical

Slow*

Fast

Reaction to photostimulation *

Provoke epileptic discharges

ncrease of epilleptic discharges

No change

$\begin{array}{lllll}0.50 & 0.51 & 0.45 & 0.56 & 0.15 \\ 0.40 & 0.55 & 0.47 & 0.79 & 0.92 \\ 0.20 & 0.22 & 0.69 & 0.72 & 0.18 \\ 0.59 & 0.68 & 0.24 & 0.51 & 0.05 \\ 0.36 & 0.64 & 0.16 & 0.46 & 0.08 \\ 0.20 & 0.40 & 0.32 & 0.50 & 0.48\end{array}$

$\begin{array}{lllll}0.77 & 0.55 & 0.28 & 0.16 & 0.05\end{array}$

$\begin{array}{lllll}0.07 & 0.08 & 0.48 & 0.46 & 0.86\end{array}$

$\begin{array}{lllll}0.05 & 0.23 & 0.04 & 0.06 & 0.05\end{array}$

$\begin{array}{lllll}0.11 & 0.14 & 0.20 & 0.33 & 0.05\end{array}$

$\begin{array}{lllll}0.21 & 0.21 & 0.21 & 0.11 & 0.16\end{array}$

$\begin{array}{lllll}0.22 & 0.11 & 0.21 & 0.19 & 0.16\end{array}$

$\begin{array}{llllll}0.17 & 0.11 & 0.09 & 0.15 & \therefore & 0.16\end{array}$

$\begin{array}{lllll}0.15 & 0.10 & 0.14 & 0.15 & 0.20\end{array}$

$\begin{array}{lllll}0.16 & 0.30 & 0.11 & 0.17 & 0.16\end{array}$

$\begin{array}{lllll}0.09 & 0.17 & 0.23 & 0.23 & 0.16\end{array}$

$\begin{array}{lllll}0.28 & 0.18 & 0.74 & 0.62 & 0.92\end{array}$

$\begin{array}{lllll}0.72 & 0.82 & 0.26 & 0.38 & 0.08\end{array}$

$\begin{array}{lllll}0.74 & 0.49 & 0.68 & 0.56 & 0.92\end{array}$

$\begin{array}{lllll}0.26 & 0.51 & 0.32 & 0.44 & 0.08\end{array}$

$\begin{array}{lllll}0.55 & 0.39 & 0.60 & 0.44 & 0.94\end{array}$

$\begin{array}{lllll}0.45 & 0.61 & 0.40 & 0.56 & 0.06\end{array}$

$\begin{array}{lllll}0.54 & 0.42 & 0.74 & 0.65 & 0.94\end{array}$

$\begin{array}{lllll}0.46 & 0.58 & 0.26 & 0.35 & 0.06\end{array}$

$\begin{array}{lllll}0.40 & 0.53 & 0.54 & 0.64 & 0.20\end{array}$

$\begin{array}{lllll}0.60 & 0.37 & 0.46 & 0.36 & 0.80\end{array}$

$\begin{array}{lllll}0.16 & 0.19 & 0.36 & 0.35 & 0.16 \\ 0.44 & 0.44 & 0.32 & 0.37 & 0.54 \\ 0.40 & 0.37 & 0.32 & 0.27 & 0.30\end{array}$




$\begin{array}{ccccc}\mathrm{sps} & \mathrm{CPS} & \mathrm{NS} & \mathrm{TCS} & \mathrm{TAS} \\ \mathrm{p}(\mathrm{s} / \mathrm{s}) & \mathrm{p}(\mathrm{s} / \mathrm{s}) & \mathrm{p}(\mathrm{s} / \mathrm{S}) & \mathrm{p}(\mathrm{s} / \mathrm{s}) & \mathrm{p}(\mathrm{s} / \mathrm{S})\end{array}$

Reaction to thyperventilation*

Provoke epileptic discharges

Increase of epileptic discharges

No change

Reaction to drowsinessisleep

Provoke epileptic discharges

Increase of epileptic discharges

No change

$\begin{array}{lllll}02 & 0.24 & 0.44 & 0.43 & 0.61 \\ 036 & 0.45 & 0.38 & 0.37 & 016 \\ 0.43 & 0.31 & 018 & 0.21 & 0.23\end{array}$

$0.30 \_0.34 \quad 0.35 \quad 0.42 \quad 0.23$

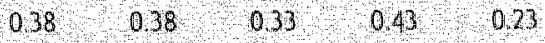

$0.32 \quad 0.27 \bullet 0.32 \quad 0.15 \quad 0.53$

* Normalized frequency estimatles. 



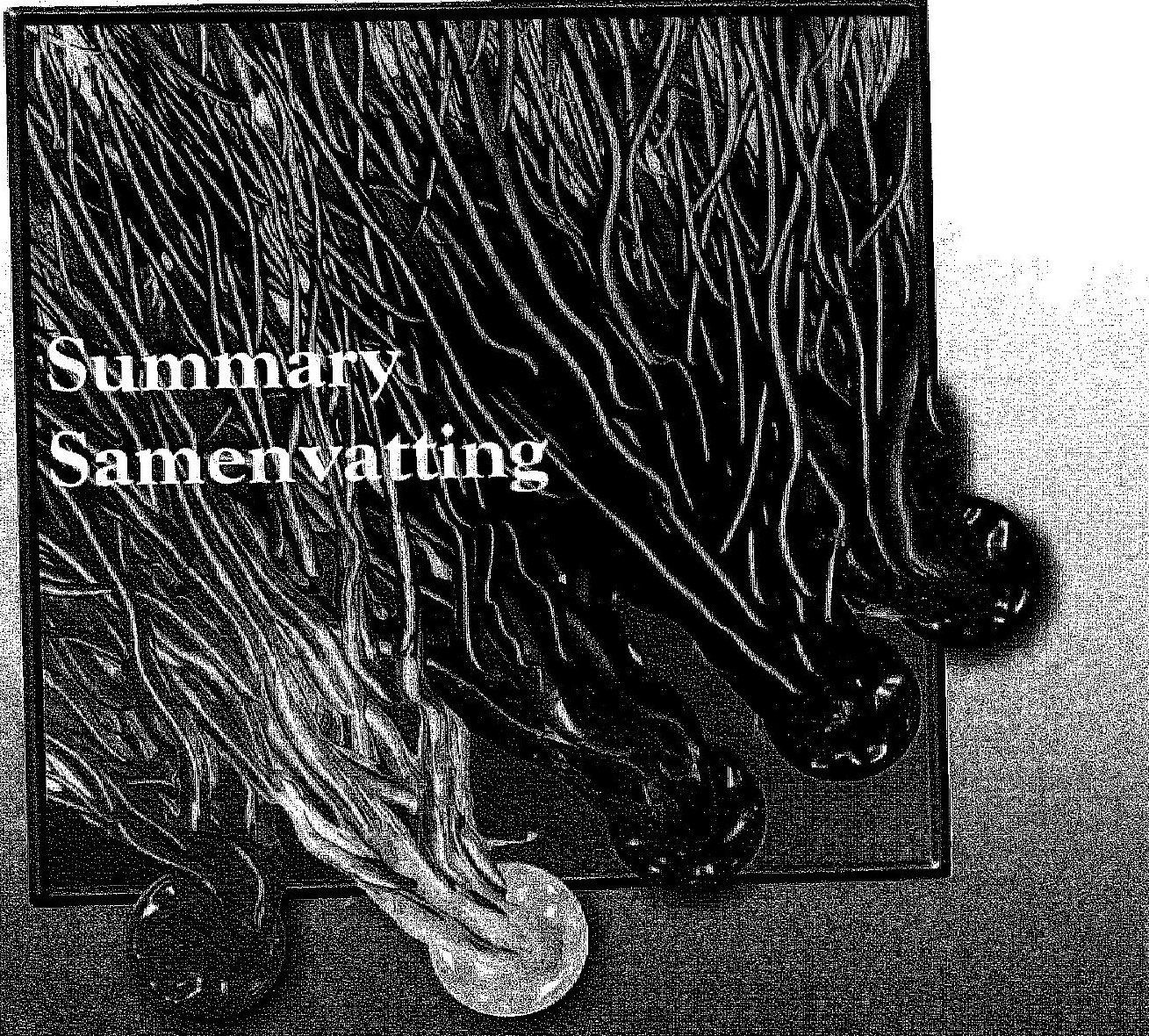


Since the 1960 w the Intemational League Against Eptlepsy (LAE) has developed several classification system for epileptic seizures. The most recent accepted Intemational Classification of Epileptic Seizures (ICES) dates from 1981. This classification prowdes rather general descriptions of the seizure cypes, which has led to variability in its use. It has been shown that the use of well defined and clearly agreed upon definitions of seizures reduces the interobserver variability in classifying seizures. Therefore we developed structured descriptions called Diagnostic Reference Frames (DRFs) by us - for specific seizure types. A DRF consists of a list of mantestations and for each manifestation an estimate of the conditional probability that such a manifestation occurs in the given seizure. These DRFs can serve as a frame of reference in the process of classifying patients with epileptic seizures. Papers that report quantitative data on manifestations or seizures in general are based on specific populations, specific subclasses of seizures types or deal with only a small set of manifestations. Therefore a meta-analysis on published resources is not feasible. We decided to base the DRFs on the knowledge and expertise of neurologists/ epileptologists. This thesis reports on the development and the validation of the knowledge included in the DRFs.

We started with a pilot study (chapter 2 and 3 ) to determine the feasibility of eliciting descriptive information on various seizure rypes from neurologists/epileptologists and to optimise the method for knowledge elicitation. In chapter 2 the inter-participant agreement in the selection of seizure manifestations for the description of the seizure types is evaluated. We found that the neurologistsfepileptologists reached a level of agreement concerning frequently occurting manifestations (manifestations present in at least $75 \%$ of the cases) that exceeded the expected agreement based on a pure random selection of manifestations. The experts selected a core of common, frequently occurring manifestations on which there was disagreement only on the core's extent.

Chapter 3 describes our study to assess whether the core of manifestations of a specific seizure rype provided by the experts differs from the cores of the other seizure types. We constructed templates for the various seizure types by counting how often each manifestation was selected by a group of participants. We matched the responses of each participant for a particular seizure with the cemplates for all seizure types based on the responses of the other participants. Also correlation coefficients were calculated between the templates. The matching scores and the correlation coefficients both show that discriminative descriptions of the seizure types could be derived fron the participants" responses. Based on the results of the pilot study we concluded that expert opinions can be used for building DRFs of seizure types. The pilot study also helped to improve the design of our main study. In the main study neurologists/epileptologists were asked to estimate the frequency of occurrence of manifestations in a particular seizure type on a 5 point scale.

Chapter 4 presents a general approach to assess whether experts involved in anowledge acquisition process provide reliable data. The reliability of the responses is determined with the inter-rater intraclass correlation coefficient (ICC). If the ICC is not large enough the Spearman-Brown prophecy formula can be used to predict the number of additional experts. We developed an approach to assess whether an additional expert provides information consistent with the already acquired data as well as a method to detect experts with deviating opinions. We applied this approach in our main knowledge elicitation procedure. The ICCs determined from the responses for the various seizure types after inclusion of 
additional neurologists/epileptologists were in all cases almost equal to 0.9, the target value. One expert with diverging opinions concerning the frequency of occurrence of manifestations for different seizure types could be identified. Excluding this participant improved the reliability of the data. We concluded that experts can provide reliable information about the frequency of occurrence of manifestations in epileptic seizures.

In the main knowledge elicitation procedure we used a Delphi (-like) procedure as to reduce the inter-observer variability. This procedure is described in chapter 5 . Ten neurologists/ epileptologists recruited in the Netherlands and fourteen at the $5^{\text {th }}$ European Epilepsy Congress estimated the frequency of occurrence of manifestations for the most frequently occurring seizure types. In the feedback round they were asked to review the manifestations for which they provided an estimate that deviated considerably from the mean estimate of the group of experts. The feedback procedure improved the reliability of the responses as measured with the ICC. By assigning a percentage value to each of the 5-points on the Likert-scale and by averaging assigned percentages we obtained an estimate of the frequency of occurrence for each manifestation for each seizure rype. The averaged frequency estimates were included in the DRFs. DRFs were developed for the most freguently occurring seizure types: simple partial seizures, complex partial seizures, myoclonic seizures, tonic-clonic seizures and typical absence seizures.

The validity of the DRFs for clinical use is evaluated and described in chapter 6. To validare the DRFs we used a decision support system based on the DRFs and using Bayes"s rule. Patient manifestations are entered in the decision support system and by successively applying Bayes's rule posterior probabilities are calculated. The DRF with the highest posterior probability gives an indication of the classification of the seizure. The validation of the DRFs was performed by comparing the seizure type with the highest posterior probability with the classification of experienced epileptologists on a series of test cases with known epileptic seizures. We included 66 patients. The patients and/or their relatives described the manifestations occurring during a seizure. Sixty cases $(91 \%)$ were correctly classified using the decision support system. The accuracy of $91 \%$ indicates that the knowledge encoded in the DRFs for the included seizure types is valid.

A prerequisite for a good classification is that manifestations are correctly observed and reported. Chapter 7 describes an observational study focussing on the quality of reporting seizure manifestations. We assessed the completeness and accuracy in reporting seizure manifestations shown on four video cases by medical students and neurology residents. Results show that the mean percentage of correct-positive manifestations reported by the participants ranged from $38.3 \%$ to $69.0 \%$. We found that this group of participants had difficulties in reporting relevant seizure manifestations. When the reported manifestations were registered in the decision support system, the system classified the same seizure as shown on the video in 20 of the 51 cases, while the participants correcrly classify the seizures in 24 of the 51 cases. This indicates that participants have observed some manifestations at an unconscious level and properly interpreted them in the diagnostic process although they did not explicitly report these manifestations. The difficulties in reporting relevant seizure manifestations of medical students and neurology residents indicate that there is a need for more clinical training in observing and reporting seizure attacks. A decision support system can be of value to support medical students and neurology residents in obtaining a complete description of the clinically relevant manifestations both affected and nor affected by the seizure and support them in classifying the seizure type. 
In chapter 8 the main findings of this thesis are summarized and the results are discussed. This thesis shows that the used methodology made it possible to develop reliable and valid DRFs for epileptic seizures based on expert opinions. These DRFs showed a diagnostic accuracy of $91 \%$ on a test set. This result seems sufficiently high to start validating the usability of the DRFs in clinical practice. Until now DRFs for five seizure types were developed. Since we concluded that medical students and neurology residents have difficulties in reporting relevant seizure manifestations and have a poor diagnostic accuracy, we suggest that a decision support system (based on the DRFs) might be offered to medical students and neurology residents during training and their residency respectively. Furthermore, the methodology is discussed and the thesis ends with recommendations for future research. 


\section{b.}

Vanaf de jaren '60 heeft de Internationale Organisatie tegen Epilepsie (ILAE) verschillende classificatiesystemen voor epileptische aanvallen on wikkeld. De meest recent geaccepreerde Internationale Classificatie voor Epileptische Aanvallen (ICES) dateert uit 1981. In deze classificatie worden de aanvalstypen over het geheel genomen algemeen beschreven. hetgeen heeft geleid tot variabiliteit in het gebruik van deze classificaties. Het is aangetoond dat het gebruik van duidelijk omschreven definities, waar overeenstemming over bestaat, leidt tot vermindering van de interobserver variabiliteit in het classificeren van aanvallen. Om deze reden hebben we gestructureerde beschrijwingen van manifestaties voor de verschillende aanvalstypen ontwikkeld. Deze beschrijwingen noemen wij Diagnostic Reference Frames (DRFs). Een DRF bestat uit een lijst met manifestaties warbij voor elke manifestatie een schatting is gegeven van de conditionele kans dat een dergelijke manifestatie voorkomt tijdens een bepaalde aanval. Deze DRFs kunnen dienen als referentie in het classificatieproces van patiënten met epileptische aanvallen. In wetenschappelijke artikelen worden wel kwantitatieve gegevens weergegeven over manifestaties of anvallen in het algemeen, maar deze gegevens zijn gebaseerd op specifieke populaties, specifieke subklassen van aanvalstypen of hebben te maken met een kleine set manifestaties. Daarom was het uitvoeren van een meta-analyse gebaseerd op gepubliceerde wetenschappelijke artikelen voor het verkrijgen van conditionele kansen per manifestatie voor de verschillende aanvalstypen niet mogelijk. We hebben besloten om de DRFs te baseren op kennis en ervaring van neurologen/epileptologen. Dit proefschrift beschrijt de ontwikkeling van de DRFs en de validatie van de kennis die opgenomen is in de DRFs.

We hebben een pilot-studie uitgevoerd (hoofdstuk 2 en 3 ) om te bepalen of het mogelijk is om beschrijvende informatie over verschillende aanvalstypen van neurologen/ epileptologen te verkrijgen en om de methode voor kenniselicitatie te optimaliseren. In hoofdstuk 2 wordt de interparticipant overeenstemming in de selectie van aanvalsmanifestaties voor de verschillende aanvalstypen geèvalueerd. De resultaten geven aan dat de neurologen/ epileptologen overeenstemming bereikten met betrekking tot frequent voorkomende manifestaties (manifestaties die in tenminste $75 \%$ van de gevallen voorkomen), waarbij de verwachte overeenstemming op bas is van een wolledig random selectie overschreden werd. De experts selecteerden een kern van overeenkomstige, frequent voorkomende man ifestaties. Alleen aan de buitenste rand van de kern verschilden de experts van mening.

Hoofdstuk 3 beschrijt de studie waarin wordt vastgesteld of de kern van manifestaties die door de experts zijn geselecteerd voor een bepaald aanvalstype verschilt van de kernen van manifestaties van de andere aanvalstypen. We ontwikkelden schema's woor de verschillende aanvalstypen door te tellen hoe vaak elke manifestatie door een groep experts werd geselecteend. Per expert zijn de antwoorden die hij/zij gaf voor een bepaald aanwalstype vergeleken met alle schema"s, die gebaseerd zijn op de antwoorden van de overige participanten. Ook zijn er tussen de schema's correlatiecoëficiènten berekend. De scores bij de vergelijking en de correlariecoëficiënten tonen beide aan dat discriminerende beschrijwingen van de aanvalstypen verkregen kunnen worden op basis van de antwootden van de participanten. Gebaseerd op de resultaten van de pilot studie concludeerden we dat de opinie wan experts gebruikt kan worden voor het ontwikkelen van DRFs woor epileptische aanvallen. De pilot studie heeft ons ook geholpen om het ontwerp van de hoofdstudie verder aan te scherpen. In de hoofdstudie wordt aan neurologen/epileptologen gevraagd om 
een schatring van de frequentie van voorkomen van manifestaties in een bepaald aanvalstype op een 5-puntsschaal te maken.

Hoofdstuk 4 beschrifft een algemene methode om vast te stellen of experts die betrokken zijn in een kennisacquisítieproces betrouwbare data verstrekken. De betrouwbaarheid van de antwoorden wordt bepaald met de interrater-intraclass-correlatiecoëfficiènt (ICC). Als de ICC niet hoog genoeg is dan kan de Spearman-Brown voorspellingsformule gebruikt worden on te berekenen hoeveel additionele experts er nodig zijn. We ontwikkelden een benadering on vast te stellen of een additionele expert informatie verstrekt die consistent is met de reeds verzamelde dara. Daanaast biedt deze benadering de mogelijkheid om experts met een afwijkende mening te achterhalen. We hebben deze benadering toegepast in de kenniselicitatieprocedure van de hoofdstudie. De ICCs, die bepaald zijn uit de antwoorden voor de verschillencle aanvalstypen nadat inclusie van de additionele neurologen/ epileptologen had plaatsgevonden, waren in alle gevallen bijna gelijk aan 0.9 , de streefwaarde. Er is éen expert geïdentificeerd die afwijkende antwoorden gaf voor wat betreft de frequentie wan woorkomen wan manifestaties voor de verschillende aanvalstypen. Uitsluiting van deze expert leidde tor een werbetering in de betrouwbaarheid van de data. We concludeerden dat experts betrouwbare informatie over de frequentie van voorkomen van manifestaties in epileptische aanvallen kunnen verschaffen.

In de kenniselicitatieprocedure tijdens de hoofdstudie hebben we gebruik gemaakt van een (semi) Delphi-procedure om de interobserver variabiliteit te reduceren. Deze procedure staat beschreven in hoofdstuk 5 . Uit Nederland namen tien neurologen/epileptologen deel aan de kenniselicitatieprocedure en tijdens het $5^{\text {de }}$ Europese Epilepsie Congres hebben nog veertien neurologen/epileptologen hun medewerking verleend. Deze neurologen/epileptologen hebben de frequentie van voorkomen van manifestaties geschat voor de meest frequent woorkomende aanvalstypen. In de feedback-ronde van de Delphi-procedure werd aan de experts gevraagd om voor manifestaties, waarvoor ze een schatting gegeven hadden die in belangrijke mate afweek van het gemiddelde van de groep experts, nogmaals een schatting te geven. De betrouwbaarheid van de schattingen, berekend met behulp van de ICC, verbeterde door de feedback-ronde. Door het toekennen van een percentage aan de 5 punten op de Likert-schaal en door het middelen wan deze toegekende percentages, hebben we schattingen wan de frequentie van voorkomen van manifestaties voor elk aanvalstype verkregen. De gemiddelde frequentie schattingen zijn opgenomen in de DRFs. Er zijn DRFs ontwikkeld woor de meest frequent voorkomende aanvalstypen: eenvoudig partielle aanvallen, complex partiële aanvallen, myoclone aanvallen, tonisch-clonische aanvallen en typische absence aanvallen.

De validiteit van de DRFs voor het gebruik in de klinische praktijk is geëvalueerd en staat beschreven in hoofdstuk 6 . Om de DRFs te valideren hebben we gebruik gemaakt van een besluitvormingsondersteunend systeem (DSS) dat gebaseerd is op de DRFs en gebruik maakt van de regel van Bayes. Manifestaties voorkomend bij patiënten worden ingevoerd in het DSS. Na iedere ingevoerde manifestatie is de regel van Bayes toegepast. Hierdoor worden de posterior schattingen berekend. Het DRF met de hoogste posterior schatting geeft een indicatie over de classificatie van de aanval. De validatie van de DRFs werd uitgevoerd door het aanvalstype met de hoogste posterior schatting te vergelijken met de classificatie van ervaren epileptologen voor een serie testcasussen waarbij het aanvalstype bekend was. We hebben 66 patiënten geincludeerd. De patiënten en/of hun familieleden hebben de manifestaties die voorkwamen tijdens de aanval beschreven. Zestig casussen 
$191 \%$ ) werden door her DSS correct geclassificeerd. Dit geeft aan dar de kennis die opgenomen is in de DRFs voor de geincludeerde aanvalstypen valide is.

Een woorwaarde voor een goede classificatie is dat manifestaties correct worden geobserveerd en gerapporteerd. In hoofdstuk 7 wordt een observationele studie beschreven waarbij gekeken wordt naar de kwaliteit van het rapporteren van anvalsmanifestaties. Wij hebben de volledigheid en de accuratesse warmee co-assistenten en assistenten in opleiding tot neuroloog manifestaties rapporteren vastgesteld aan de hand van vier videocasussen. De resultaten geven aan dat het gemiddelde percentage correct-posirief gerapporteerde manifestaries door de participanten varieert tussen de $38.3 \%$ en de $69.0 \%$ Deze groep participanten had problemen met het rapporteren van relevante aanvalsmanifestaties. Indien de gerapporteerde manifestaties voor classificatie in het DSS werden opgenomen, classificeerde het DSS in 20 van de 51 casussen dezelfde diagnose als de getoonde aanval op de videocasus, terwijl de participanten zelf 24 van de 51 casussen correct classificeerden. Dit gegeven impliceert dat de participanten sommige manifestaties onbewust hebben geobserveerd en waarschijnlijk meegenomen hebben in her diagnostisch proces alhoewel ze deze manifestaties niet expliciet gerapporteerd hebben. De problemen bij het rapporteren van relevante aanvalmanifestaties door co- en arts-assistenten geeft aan dat er behoefte is aan meer klinische training in het observeren en rapporteren van aanvallen. Een DSS kan co- en arts-assistenten ondersteunen bij het verkrijgen van een complete beschrijving van de klinisch relevante manifestaties en ondersteuning bieden in het classificeren van het aanvalstype. Bij het registreren van klinisch relevante manifestaties is het van belang om zowel de manifestaties die ontstaan als gevolg van de aanval, als de manifestaties die door de aanval niet veranderen, te tapporteren.

In hoofdstuk 8 worden de belangrijkste resultaten van dit proefschrift samengevat en besproken. Dit proefschrift toont aan dat met de gebruikte methodiek het mogelijk is om betrouwbare en valide DRFs voor epileptische aanvallen te ontwikkelen die gebaseerd zijn op opinies van experts. Deze DRFs lieten een diagnostische accuratesse van $91 \%$ zien bij een groep testcasussen. Dit resultaat lijkt voldoende om een validatie naar de toepasbaarheid van de DRFs in de klinische praktijk uit te voeren. Tot nu toe zijn er voor vijf aanvalstypen DRFs ontwikkeld. Nu we geconcludeerd hebben dat co-en arts-assistenten problemen hebben met het rapporteren van relevante aanvalsmanifestaties en een geringe diagnost tische accuratesse hebben, kan het DSS (gebaseerd op de DRFs) mogelijk gebruikt worden tijdens de opleiding van co- en arts-assistenten. Verder wordt de methodiek bediscussieerd en eindigt dit proefschrift met aanbevelingen voor toekomstig onderzoek. 



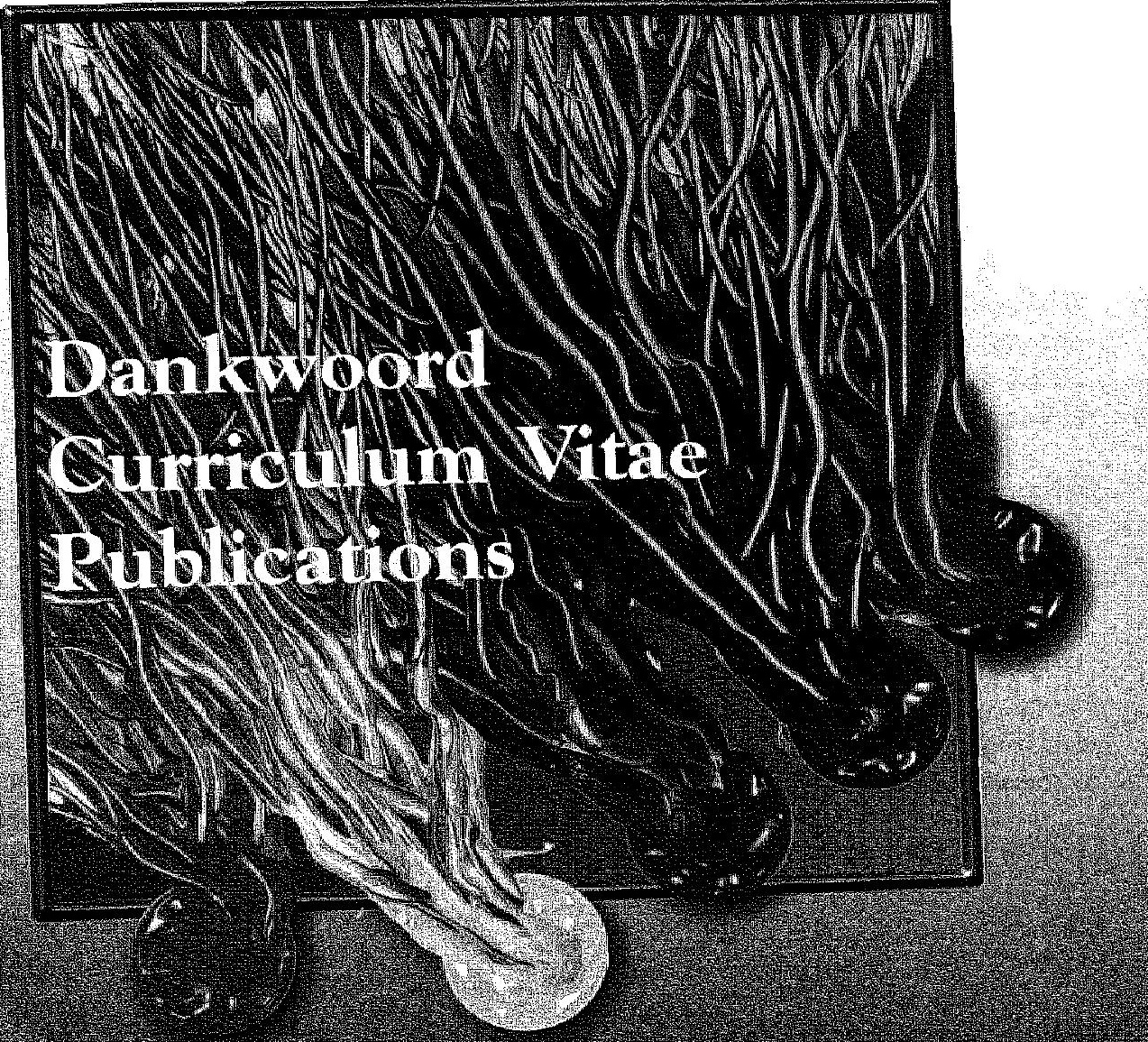




\subsection{Whan}

Het is af!

Dit proefschrift is tot stand gekomen met de medewerking van velen. Bedankt allemaal. In het bijzonder wil ik de volgende personen noemen

Arie Hasman bedankt woor je kritische kijk op het onderzoek. Vooral je vele vragen brachten mij soms in verwarring mat hebben wel tot een verdieping van de studie geleid.

Jan Talmon: bedankt woor je enthousiasme. Wanneer je een nieuw idee had wist je dit prima over te brengen. Daar hel $i k$ een hoop van geleerd.

Jan en Arie: de discussies tijdens onze projectbesprekingen waren soms eindeloos, waarbij met name jullie samen discussieerden over welke methoden wel of juist niet gebruikt moesten worden. Daarbij raakte ik de draad af en toe wel eens kwijt. Maar we zijn eruit. gekomen.

W. Renier: wanneer Jan en ik weer eens naar Nijmegen kwamen voor een bespreking ontving u ons altijd met thee/koffie en nam u ruim de rijd om alles door te nemen. Uw klinische blik heeft een wardevolle bijdrage an dit onderzoek geleverd. Mijn dank hiervoor.

Raymond Smeets \& Prof. H. Meinardi hebben met name geholpen met het opstarten van de studie. Bedankt hiervoor.

Patrick Ahles en Sjef Roos: jullie kennis van en ervaring met programmeren waren voor mij onmisbaar. Bedankt voor jullie bijdrage.

Marianne Quaden en Eugénie van Oosterhout: bedankt dat jullie mijn paranimfen willen

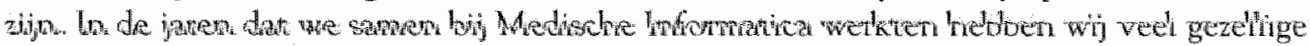
momenten gekend. Bedankt daavoor. Marianne bedankt voor alle secretariële ondersteuning.

Overige (ex-)collega's bij Medische Informatica: bedankt voor de samenwerking.

Mijn ouders: altijd weer vroegen jullie hoe het ermee stond en of jullie nog ergens mee konden helpen. Bedankt voor al deze interesse en hulp.

Ruben: vanaf april 2004 was jij er. Het zonnetje in huis.

Maurice: mijn steun en toeverlaat. Je luisterde altijd geduldig als ik thuiskwam mer de mededeling dat ik voor de zoveelste keer een artikel moest aanpassen. Het laatste jaar was niet altijd even gezellig. Vele avonden zat ik in de studeerkamer an het proefschrift te werken. Nu her proefschrift af is, kan ik in de vrije tijd die we samen hebben er weer voor jou en Ruben zijin. 


\section{THRIOULU VITAE}

Wanda van Ast werd geboren op 1 januari 1974 te Rotrerdam. In 1993 behaalde zij haar VWO-diploma aan het Mercurius College te Capelle aan den Ijssel. In datzelfde jaar startte ze met de studie HBO-Verpleegkunde aan de Hogeschool Rotterdam en Omstreken te Rotterdam, waar ze in 1997 afstudeerde. Verwolgens studeerde ze van 1997 tot 1999 Gezondheidswetenschappen aan de Universiteit Maastricht te Maastricht met als afstudeerrichting Verplegingswetenschap.

Aansluitend werkte ze als verpleegkundige in het Atrium Medisch Centrum Heerlen te Heerlen, gecombineerd met een baan als onderzoeksassistent bij de capaciteitsgroep Verplegingswetenschap aan de Universiteit Maastricht. Per 1 april 2000 startre ze voor een periode van 4 jaar als junior onderzoeker met haar promotieonderzoek bij de capaciteitsgroep Medische Informatica aan de Universiteit Maastricht. Tijdens het laatste jaar werkte ze ook mee aan het EGOOZ-project (EPD-gerelateerd onderwijs in opleidingen voor de zorg) waarin onderzoek gedaan werd naar de huidige en wenselijke EPD-gerelateerde onderwerpen binnen het (para-)medisch onderwijs in Nederland.

Vanaf 1 september 2005 is zij werkzaam bij het Interuniversitair Cardiologisch Instituut Nederland, met als standplaats de afdeling cardiologie in het Academisch Ziekenhuis Maastricht. Zij houdt zich bezig met het opzetten van een landelijke registratie van patiënten met erfelijke hartafwijkingen. 


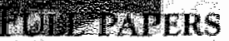

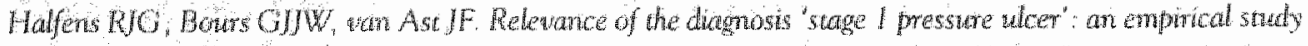

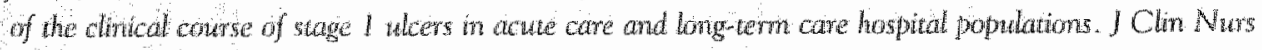
$2001,1060), 748757$

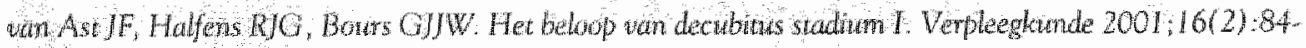
9

wath Agt JP, Talmon JL. Renier WO, Ahles PPM, Haman A. Detelopmen of diagnostic reforence franes

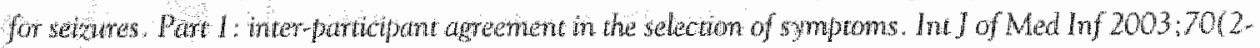
$3) 285.92$

wan Ast JF, Talmon JL, Renter WO, Meindrdi H. Ahles PPM, Haman A. Dewelopment of diagnostic referche frames for seizures. Part 2: are seitute descriptions discriminatwe? Int J of Med Inf 2003:7062 $3): 293-300$

wh Ast JF, Talmon JL. Renier WO. Hasman A. Amapmoach to knowledge base consmarton based on expert obinions. Methods Inf Med 2004:43:427-32

van Ast JF, Revier WO, Talmon JL, Roos JMA, Hasman A. Diagnostic reference frames for seizutes: A waliation Sudy. J Netrol 2005; accepted for publcation.

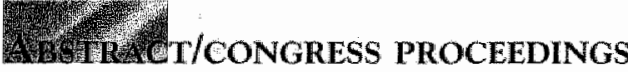

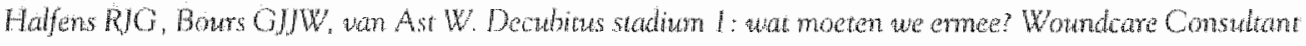
Sociedy $2001: 17(1): 25 \cdot 28$

wh Ast JF, Tamon JL. Alles PPM. Hasman A. Inerpartichant argement in the selecton of sympoms for the description of sitizues. Sud Hodth Technol inform 2002;90:644.8

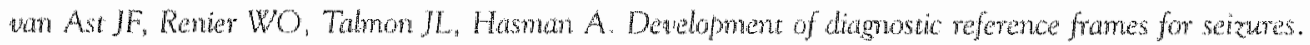

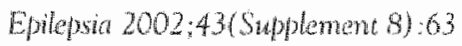

van Ast JF, Talmon JL. Reniev WO. Hasman A. How many newologaszlepileptologists are needed to prowide relable descriptions of seizure types? Stud Healh Techol Inform 2003,95:549-53 